Frey, R. (in press). Psychological drivers of individual differences in risk perception: A systematic case study focusing on 5G. Psychological Science. https://dx.doi.org/10.31234/osf.io/7a3kg

\title{
Psychological drivers of individual differences in risk perception: A systematic case study focusing on $5 \mathrm{G}$
}

\author{
Renato Frey \\ University of Basel
}

\section{Author Note}

Renato Frey, Center for Cognitive and Decision Sciences, Department of Psychology, University of Basel.

Corresponding author: Renato Frey, Department of Psychology, University of Basel, Missionsstrasse 60-62, 4055 Basel, Switzerland. E-mail: renato.frey@unibas.ch https://orcid.org/0000-0002-3190-3523 


\begin{abstract}
What drives people's perceptions of novel risks, and how malleable are such risk perceptions? Psychological science has put forth multiple drivers in this regard, but not yet tested within a unified analytic framework how well each of these account for individual differences in large population samples. To provide such a framework, the current article harnessed the deployment of $5 G$. Specifically, study 1 (representative population sample in Switzerland; $\mathrm{N}=2,919)$ conducted a multiverse analysis, finding that interindividual differences in risk perceptions were strongly associated with hazard-related (e.g., trust in the institutions regulating 5G, dread) and person-specific (e.g., electromagnetic hypersensitivity) drivers - as well as with policy-related attitudes (e.g., voting intentions). Using a longitudinal sample, study 2 conducted a field experiment based on a national expert report on 5G $(\mathrm{N}=839)$, and identified systematic links also between intraindividual changes in psychological drivers and perceived risk.
\end{abstract}

\title{
Statement of relevance
}

People's perceptions of novel risks may have far-reaching consequences in an increasingly connected world, where extreme views quickly disseminate. For policy making and targeting individuals with overly high or low risk perceptions, it is thus important to understand the psychological drivers of individual differences in risk perception. By focusing on the timely and controversial technology of $5 \mathrm{G}$ - the latest generation of cellular network technology - this article provides a comprehensive comparison of drivers that may trigger variability in risk perception between as well as within persons, and analyzes the potential downstream effects thereof on policy-related attitudes (e.g., acceptability, voting intentions). In doing so, this article identifies potential targets for future policy interventions, but at the same time illustrates that population-level changes are not easily triggered - implying that careful policy choices will be required to tap the malleability of particular drivers of risk perception.

Keywords: risk perception, individual differences, modeling, 5G, radiation 


\section{Psychological drivers of individual differences in risk perception: A systematic case study focusing on $5 \mathrm{G}$}

In the complex modern world people are confronted with novel risks at an ever-increasing pace. Some of these constitute pure "downside risks" and almost exclusively imply losses (e.g., the Covid-19 pandemic). Yet, many novel risks also reflect opportunities for individuals and society at large - as in the case of technological innovations. One such example is $5 G$, the latest generation of cellular network technology: With applications such as industrial automation, virtual reality, and next-generation transport connectivity (e.g., autonomous vehicles), 5G is expected to lead to substantial benefits globally -including an estimated contribution of up to 588 billion USD in global tax revenue from 2020 to 2034 (GSMA, 2018).

Despite these prospects, the current deployment of 5G in many countries around the world has triggered heated debates and unsettledness in the general public (Broad, 2019a; Foster, 2019). For instance, thousands of people protested against 5G in Switzerland, and many campaign for a referendum to limit radiation from mobile communications technology (Keystone, 2019a, 2019b). This uproar may come as no surprise in light of alarmist claims that appear in the media, including that 5G "might kill you" (cf. Broad, 2019b). Although such statements are typically aired by networks known for promoting fake news - "by selectively reporting the most sensational claims, and by giving a few marginal opponents of wireless technology a conspicuous new forum"- they may spread fear and undermine public trust (Broad, 2019b).

But why do some people appear to embrace this novel technology whereas others dissent? For evidence-based policy making, scientific insights into the reasons underlying people's diverging risk perceptions (which may also be at odds with experts' evaluations; Renn, 2004; Slovic, 1987) are essential in at least two respects.

First, people's risk perceptions may be strong constituents of policy-related attitudes (e.g., acceptability of risks, voting intentions). Thus, particularly when being 
propagated in social networks and the media, extreme risk perceptions of even a few people may have substantial consequences (Kasperson et al., 1988; Moussaïd et al., 2015). It is therefore important to understand who is "at risk" of adopting overly high and potentially unfounded risk perceptions (e.g., people unaware of basic technological facts). Second, it is important not only to examine which factors are relevant psychological drivers, but also to gauge their stability - and hence, the potential malleability of people's risk perceptions (Schürmann et al., 2019) — to thus identify potential targets for future policy interventions (e.g., information campaigns).

To date, psychological science has accumulated rich insights into specific drivers of risk perception. However, these drivers have not yet been compared, side-by-side, in a unified framework concerning their relevance in accounting for interindividual differences in risk perception, and intraindividual change therein. This article provides such an analytic framework in the spirit of a multiverse analysis (Steegen et al., 2016) - focusing on 5G as a timely case study - to thus address the following two research questions (RQs).

\section{What drives interindividual differences in people's risk perceptions (RQ1)?}

Interindividual differences in risk perception may emerge due to individuals' idiosyncratic (and potentially dynamic) evaluations of, or knowledge about, a specific hazard; or due to generic and relatively stable characteristics of persons themselves. The distinction between these two groups of psychological drivers is of conceptual and practical importance (e.g., in terms of potential interventions; cf. RQ2).

\section{Hazard-related drivers: Psychological evaluations of, knowledge about,}

and trust in 5G. At the forefront of psychological research investigating risk perceptions, studies implementing the psychometric paradigm (Fischhoff et al., 1978; Slovic, 1987) have examined the role of people's cognitive and affective evaluations of different hazards. Specifically, hazards that evoke high levels of dread (i.e., the involuntary exposure to hazards and uncontrollability thereof) and unknown risk (i.e., unobservable 
consequences and lack of knowledge) are perceived to be riskier, to involve fewer benefits, and hence, result in less acceptability (Slovic, 1987; Slovic et al., 1984). With a focus on comparing different hazards this approach has typically relied on average ratings of respondents - thus scaling hazards, not people. Although some research warned that average judgments of risk have to be interpreted cautiously (Vlek \& Stallen, 1981), only a few studies (Gardner et al., 1982; Pachur et al., 2012) have started to follow this advice, yet typically not implementing the full psychometric approach (but see Siegrist et al., 2005) nor recruiting large population samples. Still, dread and unknown risk are natural candidates to be considered as drivers of interindividual differences in risk perception of 5G: To the extent that a person pays attention to terrifying statements in the (social) media, or is worried about the lack of control over their exposure to radiation from network antennas, 5G will likely lead them to experience high levels of dread. Similarly, some people may perceive high levels of unknown risk, as $5 \mathrm{G}$ is a novel technology with potentially uncertain consequences (e.g., long-term effects on health).

Another line of research focusing on individual differences ex ante has identified two further factors typically related inversely to people's risk perceptions: individuals' degree of knowledge about a technology (e.g., Gstraunthaler \& Day, 2008) and trust in the authorities responsible for its regulation (e.g., Siegrist, 2000). Although trust is a multifaceted construct (Siegrist, 2019), it plays an important role in risk management (Slovic, 1993) and when people need to rely on specific institutions to reduce complexity (Siegrist, 2000, 2019). That is, particularly in the absence of sufficient knowledge about a potential hazard, trust may dampen individuals' risk perceptions (Siegrist \& Cvetkovich, 2000). Hence, in the context of $5 \mathrm{G}$ both subjective knowledge (i.e., "feeling informed") and objective knowledge (e.g., that radiation primarily results from the user's device, not the network antennas; Cousin \& Siegrist, 2010), as well as trust in the experts and institutions responsible for regulating $5 \mathrm{G}$, may attenuate individuals' risk perceptions. 
Person-specific drivers: Psychological dispositions and sociodemographic

characteristics. Interindividual differences in risk perception may also hinge on rather static factors not directly related to specific hazards, including personality dispositions, general attitudes, and sociodemographic characteristics. For instance, psychological traits

such as risk preference (Frey et al., 2017), openness to new experiences (Costa \& MacCrae, 1992), or beliefs about the advantages of digitalization may shape a person's generic preference for progression, thus potentially exerting a stable influence on the risks perceived from novel technologies. Alike, individuals who report suffering from electromagnetic hypersensitivity (EHS; i.e., non-specific symptoms associated with non-ionizing radiation; Genuis \& Lipp, 2012) may genuinely perceive any source of radiation as risky. Finally, systematic associations between sociodemographic characteristics (e.g., gender, age) and interindividual differences in risk perception have been observed (e.g., lower risk perception in young men; Finucane et al., 2000; Frey et al., 2020). Such indicators may prove useful as predictors of interindividual differences, despite not necessarily offering any direct psychological insights.

\section{How malleable are people's risk perceptions, and what drives intraindividual change (RQ2)?}

For future policy interventions it is key to understand the potential malleability of people's risk perceptions - and to identify psychological drivers that could serve as targets in this respect. Provided that people's risk perceptions primarily hinge on relatively stable, person-specific drivers, there is little hope for any malleability. The opposite is true to the extent that people's risk perceptions are shaped by hazard-related drivers (i.e., idiosyncratic evaluations of and knowledge about 5G), as these are - in principle - susceptible to change.

To date, the evidence concerning the stability of potential drivers of risk perception, and the malleability of the latter itself, is still relatively scarce - particularly in terms of longitudinal analyses, despite repeated calls in this regard (Siegrist, 2013, 2014). 
Cross-sectional results (Fox-Glassman \& Weber, 2016; Sadiq et al., 2019) suggest high stability at the aggregate level, with a pattern of rank-ordered risk perceptions of various hazards practically unchanged across almost 40 years (Fischhoff et al., 1978; Fox-Glassman \& Weber, 2016). Longitudinal results (Connor \& Siegrist, 2016; Visschers \& Siegrist, 2013) indicate that risk perceptions remain fairly stable even after incisive events such as the Fukushima accident (Visschers \& Siegrist, 2013); although fluctuations in attitudes concerning nuclear power have been observed, these were related more strongly to changes in perceived benefits rather than to changes in perceived risks (Siegrist et al., 2014).

\section{Methods}

Open research practices and ethical approval. The general project rationale and theoretical motivation, the measurement models to be implemented (including predicted patterns of associations), and the hypotheses concerning the field experiment (see study 2) were preregistered. The preregistrations, full dataset, analysis code, and additional materials can be retrieved from https://osf.io/6t3du. Ethical approval was provided by the institutional review board of the Department of Psychology, University of Basel (\#021-19-2). The full methodological details of both studies can be found in the supplemental online materials (SOM; sections A and C).

Empirical approach of study 1. Using a representative population sample in Switzerland ( $N=2,919$; representative for gender, four age groups, and the German- and French-speaking parts of Switzerland; for details see SOM A.1), study 1 conducted a systematic comparison of the state-of-the-art candidate drivers of interindividual differences in risk perception (i.e., thus addressing RQ1). The study harnessed the phase of the initial deployment of 5G in Switzerland, when public debates were fierce, culminating in some cantons (i.e., political districts) declaring 5G-moratoria - pending the conclusions of a national expert report on $5 \mathrm{G}$ to be released by the Swiss Federal Office for the Environment (Keystone, 2019a). A large heterogeneity in risk perception could thus be 
expected in the general public.

Because some of the drivers reviewed above may not be entirely independent from each other, an extensive Bayesian model comparison (i.e., multi-model inference; SOM A.3) was conducted to gauge the unique contributions of the predictors to each of three outcome variables: perceived risk, perceived benefit (i.e., personal benefits, benefits for society, benefits for the economy), and policy-related attitudes (i.e., acceptability of risks, voting intention, need for more regulation, need for more research; see SOM B.1 for the respective measurement models). Note that the treatment of variables as either predictors (i.e., "psychological drivers") or outcome variables is based on mechanisms implied by previous theory; yet, the reported effects are of correlational nature and do not necessarily reflect any causal relationships.

Empirical approach of study 2. The main purpose of study 2 was to examine the stability of the reviewed drivers, and hence gauge the potential malleability of people's risk perceptions - testing to what extent particular drivers may account not only for

\section{Figure 1}

RQ1: Drivers of interindividual differences

Study 1
(N=2,919)
Hazard-related drivers
- Dread (5G)
- Unknown risk (5G)
- Trust (5G)
- Knowledge (5G)
Person-specific drivers
- Preference for
progression
- Electromagnetic
hypersensitivity
- Age, sex, education,
occupation, ...
Outcome variables
- Perceived risk
- Perceived benefit
- Policy-related attitudes
(e.g., acceptability)

November 2019

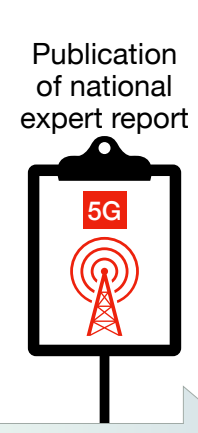

RQ2: Stability and intraindividual change

Study 2

cross-sectional

$(N=1,013)$

- Cross-validation of S1

- Mean-level change in

population (reference)

\section{Study 2}

longitudinal

$(N=839)$

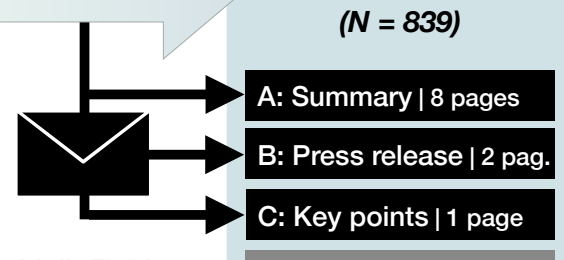

Mail: Field $\quad$ D: Control group | none experiment
January 2020

Overview of empirical approach and samples. A field experiment in the longitudinal sample implemented three naturalistic information formats of the expert report. 
interindividual differences in large population samples (RQ1), but also for intraindividual change therein (RQ2). Study 2 collected a cross-sectional sample (to validate study 1 and model mean-level changes in the population, $N=1,013$ ) and a longitudinal sample (to model intraindividual change, $N=839$; see SOM C.1 for a detailed description of the sample recruitment), approximately three months after study 1 , and after release of a national expert report on 5G (see Fig. 1 for an overview). This report (SOM C.3) was published by the Swiss Federal Office for the Environment and explained technical facts about 5G, dealt with the operation of the Swiss mobile radio networks and their regulation, estimated the population's exposure to radiation, and summarized the scientific findings on health consequences. The report did not conclude with a single recommendation; rather, it sketched multiple scenarios and served as an evidence base for informing the general public and policy making in the Swiss government. In the longitudinal sample, participants were randomly assigned to one of four conditions (i.e., field experiment; Fig. 1). Specifically, between the two studies participants of groups A-C received excerpts from the expert report by mail (using different information formats; SOM C.3) and participants of group D served as controls.

\section{Results}

\section{Study 1: Drivers of interindividual differences (RQ1)}

Main outcome variables. The majority of respondents (65\%) perceived the risks of $5 \mathrm{G}$ as medium to high (i.e., a rating $>50$ on the scale ranging from 0 to 100), and the majority (65\%) perceived low to medium personal benefits. Conversely, the majority of respondents perceived medium to high benefits for society and for the economy (61\% and $76 \%$ of respondents). As to be expected given the fierce public debates, there was substantial heterogeneity in perceived risk and benefit across respondents (Fig. S1, upper panel).

In terms of policy-related attitudes (Fig. S1, lower panel), a slight majority of 
respondents (57\%) perceived the acceptability of the potential risks as low to medium but the clear majority perceived a need for more regulation and for more research $(74 \%$ and $90 \%$ of respondents). In the event of a national referendum, about half of the respondents $(52 \%)$ would vote against $5 \mathrm{G}$. There was again strong heterogeneity across respondents.

Associations of psychological drivers with outcome variables. Figure 2 shows the distributions of all continuous predictors (see SOM B.1 for the respective measurement models) and their zero-order correlations with perceived risk. To robustly estimate and directly compare the effects of these predictors and those of three noncontinuous predictors (gender and occupation: nominal; education: ordinal), and to gauge their relative contributions in predicting the three outcome variables, exhaustive Bayesian multi-model inference analyses (SOM A.3) were conducted using the R-package $B A S$ (Clyde et al., 2011). Specifically, all possible model combinations were assembled by including or excluding the 11 predictors as linear additive effects, resulting in $2^{11}=2,048$ models. For policy-related attitudes, perceived risk and benefit were additionally considered as direct predictors, resulting in $2^{13}=8,192$ models. Note that the reporting of political attitude was optional (SOM A.2) and would have resulted in 488 list-wise deletions; given its low zero-order correlation with perceived risk (Fig. 2), this variable was

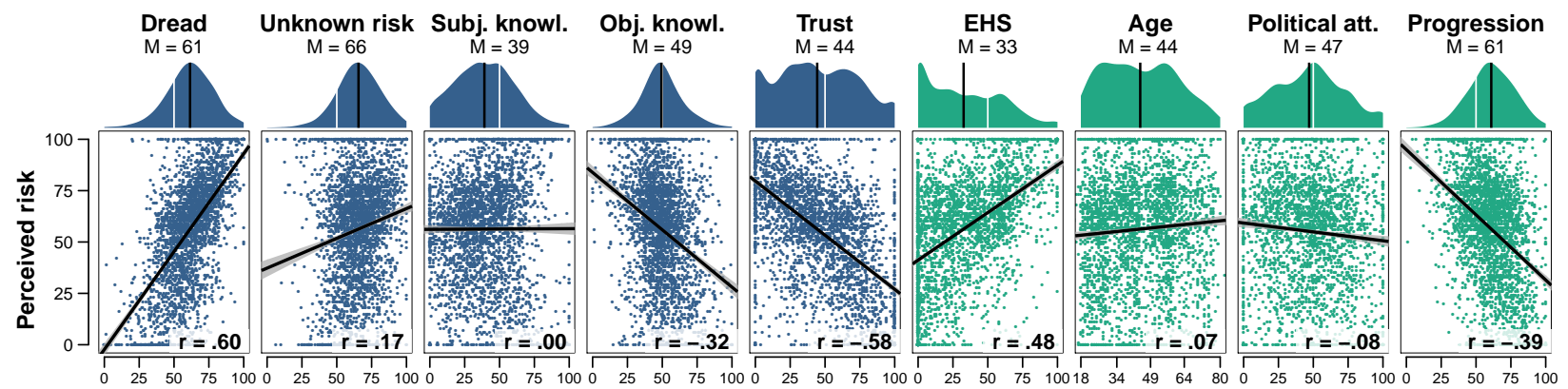

\section{Figure 2}

Distributions of potential drivers of interindividual differences in risk perception (blue: hazard-related drivers; green: person-specific drivers) and their associations with risk perception of $5 G$ in study $1(N=2,919)$. Black vertical lines represent the means (M) of the distributions. Predictors modeled as latent variables in the main analysis (e.g., dread) are depicted as approximative scores on the original response scale (SOM B.1). EHS = Electromagnetic hypersensitivity. 
excluded from this analysis. All of the Bayes Factors reported below are logarithmized with base 10 and reflect the relative evidence of the best model over the same model without the respective predictor (or vice versa if the predictor was not part of the best model).

As can be seen from the model-averaged estimates depicted in Figure 3 (panel A), dread of 5G and EHS were positive predictors of perceived risk, whereas respondents' trust in the authorities regulating $5 \mathrm{G}$, gender (male), objective knowledge about $5 \mathrm{G}$, and a respondent's generic preference for progression were inverse predictors of perceived risk. The three best predictors of perceived risk were trust with a Bayes Factor (BF) of 69, dread with a BF of 61, and EHS with a BF of 54 (Fig. 3, panel D). All of these BFs represent "very strong" evidence (Raftery, 1995) that these drivers contribute substantially to predicting perceived risk. For objective knowledge about 5G, preference for progression, and gender there was also "very strong" evidence, yet with BFs of 16, 8, and 6 considerably

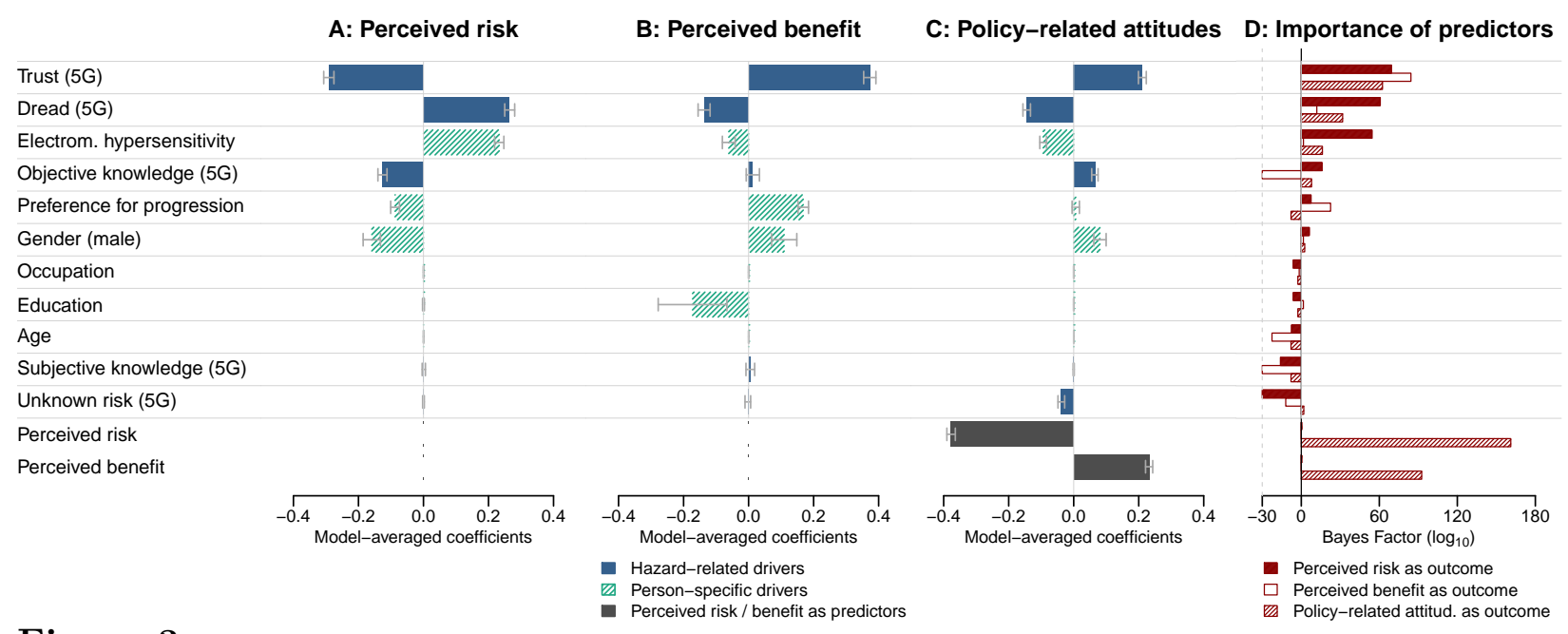

Figure 3

Results of the Bayesian multi-model inference analyses in study 1 ( $N=2,917$, after two list-wise deletions due to missing values). Panels $A-C$ depict model-averaged coefficients for all predictors, and separately for the three main outcome variables (for policy-related attitudes, perceived risk and benefit were included as direct predictors; panel C). Coefficients are standardized, and error bars depict standard deviations across models. Panel D depicts each predictor's contribution to explaining interindividual differences in the three outcome variables in terms of $\log _{10}$-Bayes Factors. Bayes Factors reflect the relative evidence of the best model over the same model without the respective predictor (or vice versa if the predictor was not part of the best model). 
less so as compared to the first three predictors. For the remaining predictors there was less or even negative evidence, indicating no meaningful contributions to predicting perceived risk (Fig. 3, panel D).

For perceived benefits (Fig. 3, panel B), the pattern of associations was very similar but essentially inverse. Yet, trust had an even stronger effect (as compared to for perceived risk) and constituted the most important predictor $(\mathrm{BF}=84)$, followed by a respondent's preference for progression $(\mathrm{BF}=23)$ and dread $(\mathrm{BF}=12$; all BFs reflect "very strong" evidence). Finally, for policy-related attitudes (Fig. 3, panel C), the associations were again similar to those for perceived risk - but again inverse. Perceived risk and benefit, which were included as direct predictors in this analysis, were the two most important predictors with BFs of 161 and 93, followed by trust $(\mathrm{BF}=62)$, dread $(\mathrm{BF}=32)$, and EHS $(\mathrm{BF}=16)$.

\section{Study 2: Stability and intraindividual change (RQ2)}

Cross-validation and population-level change. As a robustness check, the analyses of study 1 were replicated in the cross-sectional sample of study 2 and clearly corroborated the results (SOM D.1). This sample was also used to gauge any mean-level changes in the population (e.g., due to the publication of the expert report and the associated media coverage); yet, by and large there were no overall differences for perceived risk and benefit, nor for policy-related attitudes (SOM D.2).

Intraindividual change in the psychological drivers. Figure 4 (lower panel) depicts the distributions of intraindividual change (i.e., from study 1 to study 2 ) in the various hazard-related drivers, separately for the four experimental conditions. The degree of intraindividual change (i.e., $\Delta$ ) - relative to the variability that occurred between individuals - was quantified by means of intraclass-coefficients (ICCs; an ICC of 1 represents no intraindividual variability, an ICC of 0 represents pure intra- and no interindividual variability, and an ICC of .5 represents the same degree of variability 
between as within individuals; one-way random, single score $\operatorname{ICC}(1,1)$ were used). The ICCs indicated that there was a considerable degree of intraindividual change for most of the hazard-related drivers, as can also be seen from the dispersions of the distributions shown in Figure 4. To illustrate, in the control group the ICCs for unknown risk and objective knowledge were as low as .55, and for several of the investigated drivers intraindividual change tended to be even larger in the three treatment groups (e.g., an even lower ICC of .39 for objective knowledge in the group that received the press release for the expert report).

As Figure 4 illustrates, the emerged intraindividual changes involved increases and decreases (i.e., differential effects across respondents), and the respective distributions were

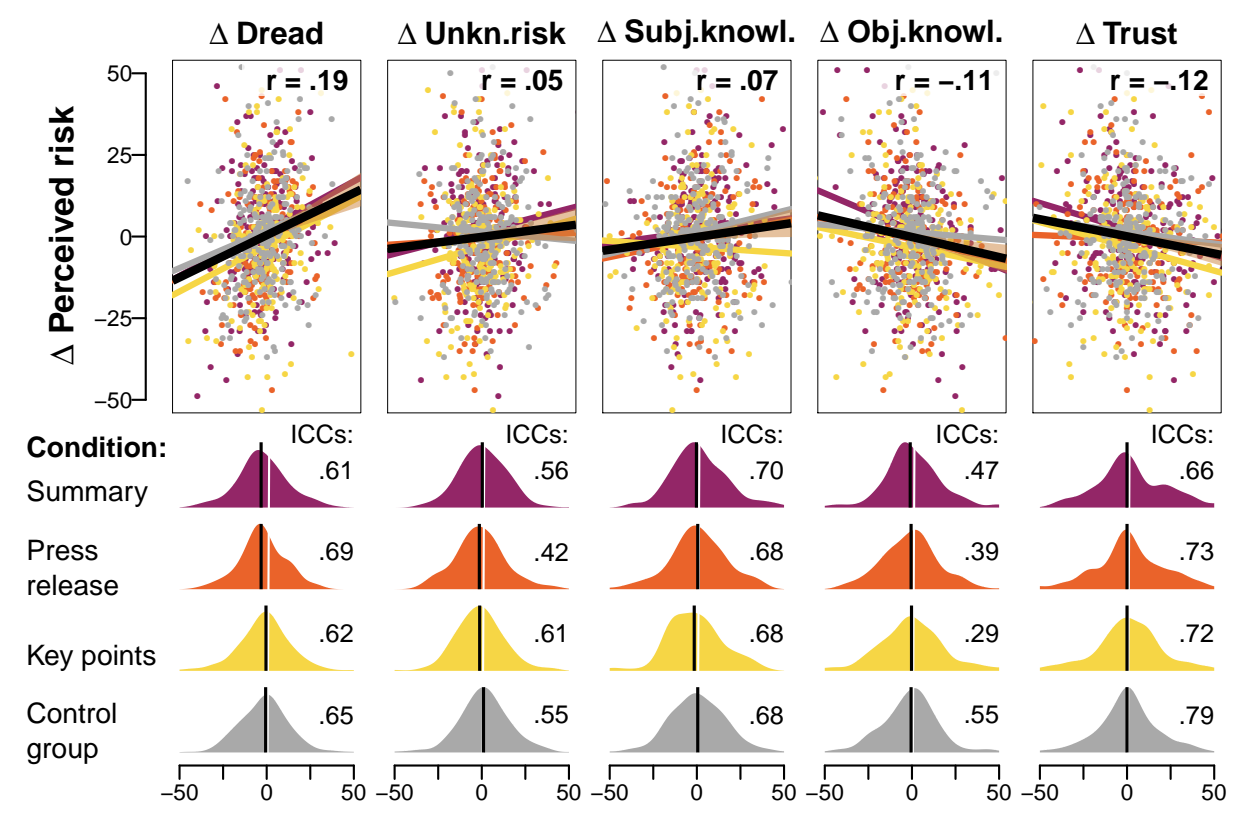

\section{Figure 4}

Distributions of intraindividual change from study 1 to study $2(\Delta)$ in the hazard-related drivers (separately for the four experimental conditions) and respective associations with intrandividual change in perceived risk (longitudinal sample; $N=839$ ). Black vertical lines represent the means of the distributions of intraindividual change. Between the two studies, participants of the treatment conditions received excerpts of the national expert report on 5G by mail (8-page summary; 2-page press release; 1 page with key points); participants of the control group received no information (see Fig. 1). Predictors modeled as latent variables in the main analysis (e.g., dread) are depicted as approximative scores on the original response scale (SOM B.1). ICCs = Intraclass-coefficients. 
centered close to zero. This implies that no pronounced mean-level changes occurred in most of these drivers or across the four experimental conditions. One exception consisted of weak but credible decreases in dread in all conditions except condition C (i.e., four key points of the expert report); namely, a mean decrease of $-2.2(95 \% \mathrm{HDI}=-3.9$ to -.6$)$ in condition A (i.e., summary of the expert report), a mean decrease of -2.3 (95\% HDI $=-4.3$ to -.7 ) in condition B (i.e., press release of the expert report), and a mean decrease of -1.7 $(95 \%$ HDI $=-3.1$ to -.04$)$ in condition D (control group).

By definition, person-specific drivers are relatively (e.g., personality dispositions) or entirely (e.g., gender) stable. Thus, as to be expected there was no or only little intraindividual variability in most of these drivers (e.g., for age and political attitude, ICCs were 1 and .88; SOM D.3). For this reason, person-specific drivers are not shown in Figure 4 and were not examined in this context.

Intraindividual change in the outcome variables. There was also a considerable degree of intraindividual change in most of the outcome variables (Fig. S11) - albeit to a somewhat smaller degree as compared to the various hazard-related drivers. To illustrate, the ICCs for perceived risk were .73, .71, .77, and .76 in the four experimental conditions, respectively. Moreover, the distributions of intraindividual change in perceived risk, perceived benefit, and policy-related attitudes were also centered close to zero, suggesting that no pronounced mean-level changes occurred overall, nor as a function of the various experimental conditions. The few exceptions consisted of condition A (i.e., summary of the expert report), which resulted in credible increases in perceived benefit for society (mean increase $=3.9,95 \% \mathrm{HDI}=1.3$ to 6.8 ) and perceived benefit for the economy ( mean increase $=2.8,95 \% \mathrm{HDI}=0.2$ to 5.5 ). Moreover, in condition C (i.e., four key points of the expert report) there was a credible increase in the acceptability of the potential risks (mean increase $=2.8,95 \% \mathrm{HDI}=0.2$ to 5.7 ).

Associations of intraindividual change in the psychological drivers with intraindividual change in the outcome variables. Figure 4 (upper panel) depicts 
the associations of intraindividual change in the various hazard-related drivers with intraindividual change in perceived risk (i.e., the main outcome variable). As can be seen, $\Delta$-dread, $\Delta$-unknown risk, and $\Delta$-subjective knowledge tended to be positively associated with intraindividual change in perceived risk, whereas $\Delta$-objective knowledge and $\Delta$-trust tended to be inversely associated with intraindividual change in perceived risk.

To systematically test whether intraindividual changes in any of the psychological drivers were reliably associated with intraindividual changes in the outcome variables (i.e., perceived risk, perceived benefit, and policy-related attitudes), the equivalent multi-model inference analyses of study 1 were conducted, yet using the $\Delta$ s of predictor and outcome variables. According to this analysis, $\Delta$-dread ( $\mathrm{BF}=1.4$; "strong" evidence) and $\Delta$-trust $(\mathrm{BF}=.2$; "weak" evidence) were reliable predictors of intraindividual change in perceived risk (Fig. S12). Note that compared to study 1 BFs generally tended to be lower, at least in part due to the smaller size of the longitudinal sample. For intraindividual change in perceived benefit, there were reliable positive associations with $\Delta$-trust and $\Delta$-objective knowledge (BFs = 3.9 and .9; "very strong" and "positive" evidence), and a weak inverse association with $\Delta$-unknown risk ( $\mathrm{BF}=1.0$; "positive" evidence). Finally, for intraindividual change in policy-related attitudes, $\Delta$-perceived risk was the best predictor (inverse association) with a BF of 15.0 ("very strong" evidence), followed by $\Delta$-perceived benefit (positive association) with a BF of 4.5 ("very strong" evidence).

\section{Discussion}

The unifying analytic framework of this article permitted, for the first time, the integration of a set of psychological drivers that were put forth by state-of-the-art theories, thus clarifying their role in shaping individual differences in risk perception. Specifically, an extensive Bayesian model comparison - conducted in the spirit of multiverse analyses aimed at increasing the transparency and robustness of psychological research (Steegen et al., 2016) — revealed that three hazard-related drivers stood out concerning their 
associations with interindividual differences in risk perceptions: Dread of 5G was strongly associated with higher risk perceptions, whereas trust in the institutions regulating $5 \mathrm{G}$ and objective knowledge about 5G were strongly associated with lower risk perceptions. Two more generic, person-specific drivers (electromagnetic hypersensitivity and gender) contributed as further predictors of interindividual differences. Crucially, changes in risk perception that occurred within individuals across time were primarily associated with intraindividual changes in dread and trust, whereas changes in perceived benefit were primarily associated with intraindividual changes in trust and objective knowledge. Finally, people's policy-related attitudes proved to hinge strongly on perceived risk (inversely) and benefit (positively), as well as to be sensitive to respective changes therein across time.

Although still other drivers of risk perception could be considered (e.g., world views; Peters \& Slovic, 1996) and future research should validate the current findings in other contexts, the present results provide a solid empirical fundament for future theory development (e.g., by identifying relevant psychological drivers to be studied in more detail in future research) as well as for policy making. Specifically, the current analyses indicated strong differences in people's risk perceptions of $5 \mathrm{G}$, and a number of psychological drivers accounted well for this heterogeneity in the general public. These observations highlight the need for taking into account individual differences when accounting for people's risk perceptions. Moreover, the observed intraindividual changes in hazard-related drivers imply some malleability of people's risk perceptions. That is, if the goal is to mitigate overly high risk perceptions of $5 \mathrm{G}$, a promising approach may consist of establishing public trust, fostering people's knowledge, and (hence) reducing feelings of dread.

In light of the alarmist statements and persistent fake news in the (social) media (Broad, 2019b), this may not be an easy task, however: The current studies demonstrated that substantial population-level effects are not readily triggered, at least not with the relatively "mild" interventions implemented here (i.e., the primary purpose of the national expert report was of an informational nature). In addition to designing stronger and more 
specific population-level interventions, in future policy initiatives it may prove useful to also consider targeted interventions, given that the field experiment revealed differential effects across respondents. The longitudinal analyses identified which drivers showed intraindividual variability (e.g., trust, objective knowledge, dread), and these could thus be instrumental to this end. Although it may be an effortful process for policy makers to influence drivers such as trust - which is typically created slowly (Slovic, 1993) - this effort could ultimately pay off, as trust may be less fragile (Siegrist, 2019) than initially thought (Slovic, 1993).

As a final word of caution, this article does not postulate that high risk perceptions are inherently undesirable. For societal welfare, critical voices will be essential in public debates, particularly in countries with high levels of participatory democracy (e.g., Switzerland, USA; Slovic, 1993). Yet, if alarmist statements and fake news trigger excess fear and undermine public trust, policy action may be required to reconcile lay people's conflicting risk perceptions - and this article provides evidence-based insights to facilitate this process.

\section{Author Contributions}

R.F. designed and performed research, conducted the data analysis, and wrote the paper.

\section{Acknowledgments}

This work was supported by the Swiss Federal Office for the Environment and a grant of the Swiss National Science Foundation (PZ00P1_174042). I am grateful to Eva Günther, the members of the Center for Cognitive and Decision Sciences and the Ambizione team, Elisabeth Lauper Orth, and Maurane Riesen for providing valuable feedback, and to Laura Wiles for proofreading the manuscript. 


\section{References}

Broad, W. J. (2019a). The 5G health hazard that isn't [newspaper]. The New York Times. Retrieved November 4, 2019, from https://www.nytimes.com/2019/07/16/science/5g-cellphones-wireless-cancer.html

Broad, W. J. (2019b). Your 5G phone won’t hurt you. But Russia wants you to think otherwise. [newspaper]. The New York Times: Science. Retrieved June 19, 2020, from https://www.nytimes.com/2019/05/12/science/5g-phone-safety-health-russia.html

Clyde, M. A., Ghosh, J., \& Littman, M. L. (2011). Bayesian adaptive sampling for variable selection and model averaging. Journal of Computational and Graphical Statistics, 20 (1), 80-101. https://doi.org/10.1198/jcgs.2010.09049

Connor, M., \& Siegrist, M. (2016). The stability of risk and benefit perceptions: A longitudinal study assessing the perception of biotechnology. Journal of Risk Research, 19(4), 461-475. https://doi.org/10.1080/13669877.2014.988169

Costa, P. T., \& MacCrae, R. R. (1992). Revised NEO personality inventory (NEO PI-R) and NEO five-factor inventory (NEO-FFI): Professional manual. Psychological Assessment Resources, Incorporated.

Cousin, M.-E., \& Siegrist, M. (2010). The public's knowledge of mobile communication and its influence on base station siting preferences. Health, Risk $\mathcal{E}$ Society, 12(3), 231-250. https://doi.org/10.1080/13698571003710332

Finucane, M. L., Slovic, P., Mertz, C. K., Flynn, J., \& Satterfield, T. A. (2000). Gender, race, and perceived risk: The 'white male' effect. Health, Risk \&5 Society, 2(2), 159-172. https://doi.org/10.1080/713670162

Fischhoff, B., Slovic, P., Lichtenstein, S., Read, S., \& Combs, B. (1978). How safe is safe enough? A psychometric study of attitudes towards technological risks and benefits. Policy Sciences, 9(2), 127-152. https://doi.org/10.1007/BF00143739 
Foster, K. R. (2019). 5G is coming: How worried should we be about the health risks? [newspaper]. Scientific American Blog Network. Retrieved November 4, 2019, from https://blogs.scientificamerican.com/observations/5g-is-coming-how-worriedshould-we-be-about-the-health-risks/

Fox-Glassman, K. T., \& Weber, E. U. (2016). What makes risk acceptable? Revisiting the 1978 psychological dimensions of perceptions of technological risks. Journal of Mathematical Psychology, 75, 157-169. https://doi.org/10.1016/j.jmp.2016.05.003

Frey, R., Pedroni, A., Mata, R., Rieskamp, J., \& Hertwig, R. (2017). Risk preference shares the psychometric structure of major psychological traits. Science Advances, 3 , e1701381. https://doi.org/10.1126/sciadv.1701381

Frey, R., Richter, D., Schupp, J., Hertwig, R., \& Mata, R. (2020). Identifying robust correlates of risk preference: A systematic approach using specification curve analysis. Journal of Personality and Social Psychology. https://doi.org/10.1037/pspp0000287

Gardner, G. T., Tiemann, A. R., Gould, L. C., Deluca, D. R., Doob, L. W., \& Stolwijk, J. A. J. (1982). Risk and benefit perceptions, acceptability judgments, and self-reported actions toward nuclear power. The Journal of Social Psychology, 116(2), 179-197. https://doi.org/10.1080/00224545.1982.9922770

Genuis, S. J., \& Lipp, C. T. (2012). Electromagnetic hypersensitivity: Fact or fiction? Science of The Total Environment, 414, 103-112. https://doi.org/10.1016/j.scitotenv.2011.11.008

GSMA. (2018). Study on socio-economic benefits of 5G services provided in mmwave bands. https://www.gsma.com/spectrum/resources/mmwave-5g-benefits/

Gstraunthaler, T., \& Day, R. (2008). Avian influenza in the UK: Knowledge, risk perception and risk reduction strategies. British Food Journal, 110(3), 260-270. https://doi.org/10.1108/00070700810858673 
Kasperson, R. E., Renn, O., Slovic, P., Brown, H. S., Emel, J., Goble, R., Kasperson, J. X., \& Ratick, S. (1988). The social amplification of risk: A conceptual framework. Risk Analysis, 8(2), 177-187. https://doi.org/10.1111/j.1539-6924.1988.tb01168.x

Keystone. (2019a). Opponents of 5G start campaign to force nationwide vote [newspaper]. SWI swissinfo.ch. Retrieved November 5, 2019, from https://www.swissinfo.ch/eng/politics/direct-democracy_opponents-of-5g-startcampaign-to-force-nationwide-vote/45299102

Keystone. (2019b). Several thousand protest against 5G in Swiss capital [newspaper]. SWI swissinfo.ch. Retrieved November 5, 2019, from https://www.swissinfo.ch/eng/society/radiation-fears_several-thousand-protestagainst-5g-in-swiss-capital/45246224

Moussaïd, M., Brighton, H., \& Gaissmaier, W. (2015). The amplification of risk in experimental diffusion chains. Proceedings of the National Academy of Sciences, 201421883. https://doi.org/10.1073/pnas.1421883112

Pachur, T., Hertwig, R., \& Steinmann, F. (2012). How do people judge risks: Availability heuristic, affect heuristic, or both? Journal of Experimental Psychology: Applied, 18(3), 314-330. https://doi.org/10.1037/a0028279

Peters, E., \& Slovic, P. (1996). The role of affect and worldviews as orienting dispositions in the perception and acceptance of nuclear power. Journal of Applied Social Psychology, 26(16), 1427-1453. Retrieved April 22, 2014, from http://onlinelibrary.wiley.com/doi/10.1111/j.1559-1816.1996.tb00079.x/full

Raftery, A. E. (1995). Bayesian model selection in social research. Sociological Methodology, 25, 111-163. https://doi.org/10.2307/271063

Renn, O. (2004). Perception of risks. Toxicology Letters, 149(1-3), 405-413. https://doi.org/10.1016/j.toxlet.2003.12.051 
Sadiq, A.-A., Tharp, K., Graham, J. D., \& Tyler, J. (2019). Temporal stability and changes in risk perception rankings of hazardous activities and technologies. Journal of Risk Research, 22(1), 93-109. https://doi.org/10.1080/13669877.2017.1351474

Schürmann, O., Frey, R., \& Pleskac, T. J. (2019). Mapping risk perceptions in dynamic risk-taking environments. Journal of Behavioral Decision Making, 32, 94-105. https://doi.org/10.1002/bdm.2098

Siegrist, M. (2000). The influence of trust and perceptions of risks and benefits on the acceptance of gene technology. Risk Analysis, 20 (2), 195-204. https://doi.org/10.1111/0272-4332.202020

Siegrist, M. (2013). The necessity for longitudinal studies in risk perception research. Risk Analysis, 33(1), 50-51. https://doi.org/10.1111/j.1539-6924.2012.01941.x

Siegrist, M. (2014). Longitudinal studies on risk research. Risk Analysis, 34 (8), 1376-1377. https://doi.org/10.1111/risa.12249

Siegrist, M. (2019). Trust and risk perception: A critical review of the literature. Risk Analysis. https://doi.org/10.1111/risa.13325

Siegrist, M., \& Cvetkovich, G. (2000). Perception of hazards: The role of social trust and knowledge. Risk Analysis, 20(5), 713-720. https://doi.org/10.1111/0272-4332.205064

Siegrist, M., Keller, C., \& Kiers, H. A. L. (2005). A new look at the psychometric paradigm of perception of hazards. Risk Analysis, 25(1), 211-222. https://doi.org/10.1111/j.0272-4332.2005.00580.x

Siegrist, M., Sütterlin, B., \& Keller, C. (2014). Why have some people changed their attitudes toward nuclear power after the accident in Fukushima? Energy Policy, 69, 356-363. https://doi.org/10.1016/j.enpol.2014.02.026

Slovic, P. (1987). Perception of risk. Science, 236(4799), 280-285. https://doi.org/10.1126/science.3563507 
Slovic, P. (1993). Perceived risk, trust, and democracy. Risk Analysis, 13(6), 675-682. https://doi.org/10.1111/j.1539-6924.1993.tb01329.x

Slovic, P., Fischhoff, B., \& Lichtenstein, S. (1984). Behavioral decision theory perspectives on risk and safety. Acta Psychologica, 56(1-3), 183-203. https://doi.org/10.1016/0001-6918(84)90018-0

Steegen, S., Tuerlinckx, F., Gelman, A., \& Vanpaemel, W. (2016). Increasing transparency through a multiverse analysis. Perspectives on Psychological Science, 11(5), 702-712. https://doi.org/10.1177/1745691616658637

Visschers, V. H. M., \& Siegrist, M. (2013). How a nuclear power plant accident influences acceptance of nuclear power: Results of a longitudinal study before and after the Fukushima disaster. Risk Analysis, 33(2), 333-347. https://doi.org/10.1111/j.1539-6924.2012.01861.x

Vlek, C., \& Stallen, P.-J. (1981). Judging risks and benefits in the small and in the large. Organizational Behavior and Human Performance, 28(2), 235-271. https://doi.org/10.1016/0030-5073(81)90024-6 


\title{
Supplemental Online Materials
}

Psychological drivers of individual differences in risk perception:

A systematic case study focusing on $5 \mathrm{G}$

\author{
Renato Frey \\ University of Basel
}

February 3, 2021 


\section{Contents}

A Detailed methods study $1 \quad 3$

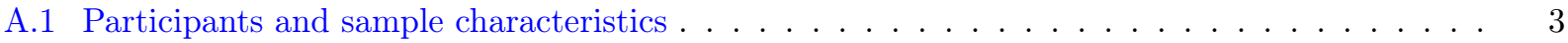

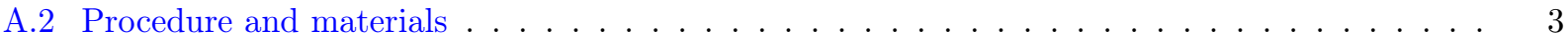

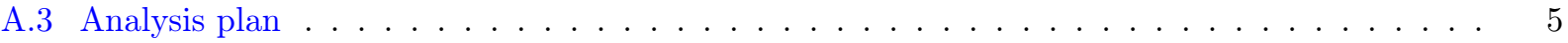

A.4 Deviations from preregistered analysis $\mathrm{plan} \ldots \ldots \ldots \ldots \ldots$

B Extended results study $1 \quad 5$

B.1 Measurement models . . . . . . . . . . . . . . . . . . . . . . . . . 5 5

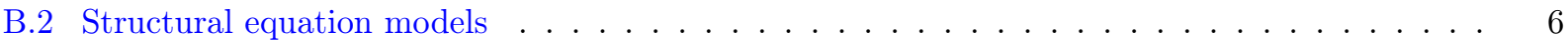

B.3 GIS analysis of network coverage $\ldots \ldots \ldots \ldots \ldots \ldots \ldots \ldots \ldots \ldots$

B.4 Perception of health risks $\ldots \ldots \ldots \ldots \ldots \ldots$

$\begin{array}{ll}\text { C Detailed methods study } 2 & 8\end{array}$

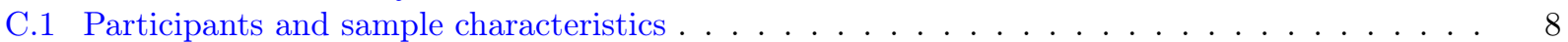

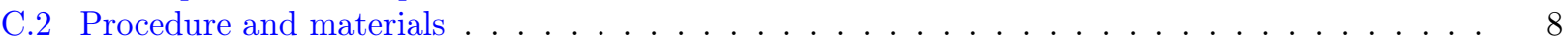

C.3 Expert report on $5 \mathrm{G}$ and experimental conditions in the longitudinal sample $\ldots \ldots \ldots$

C.4 Analysis plan . . . . . . . . . . . . . . . . . . . . . . . 9

$\begin{array}{ll}\text { D Extended results study } 2 & 10\end{array}$

D.1 Cross-validations of study 1 (cross-sectional sample) $\ldots \ldots \ldots \ldots \ldots$

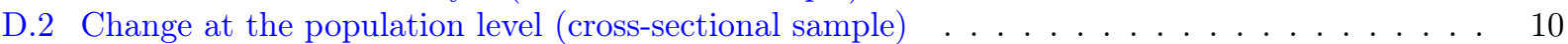

D.3 Intraindividual change in person-specific drivers (longitudinal sample) . . . . . . . . . . . 10

D.4 Associations of intraindividual change in predictor and outcome variables (longitudinal sample) 11

$\begin{array}{llr}\text { E } & \text { Figures } & 12\end{array}$

F Tables 


\section{A Detailed methods study 1}

The institutional review board of the Department of Psychology, University of Basel provided ethical approval (\#021-19-2). The general project rationale and theoretical motivation, the models to be implemented including predicted patterns of associations, and the hypotheses concerning the field experiment (see study 2 ) were preregistered. The preregistrations, dataset, analysis code, and additional materials can be retrieved from https://osf.io/6t3du.

\section{A.1 Participants and sample characteristics}

Study 1 was conducted in November 2019 and involved a large sample of the Swiss general public, representative for gender, four age groups (15-29 years; 30-44 years; 45-59 years; 60-79 years), and two regions (German- and French-speaking parts of Switzerland). Participant recruitment was performed by an external survey company using an existing panel, which exclusively comprised participants who were actively recruited by telephone (i.e., no self-registrations possible). Sampling was conducted using interlocked quotas and slightly exceeded the aspired sample size of 3,000 participants, resulting in $N_{S 1}=3,096$ participants who completed the study. Participants who failed an attention check question (see A.2) were excluded from the dataset, resulting in a final sample of 2,919 participants. Table S1 reports sociodemographic information. Data collection was completed on November 26, 2019; that is, before release of the expert report on 5G by the Swiss Federal Office for the Environment (see C.3). On average it took participants 14 minutes to complete the study; as a compensation, participants earned 100 points (i.e., equivalent to $1 \mathrm{CHF}$ ) in the survey company's incentive program.

\section{A.2 Procedure and materials}

The study was conducted online using a responsive mobile first design (i.e., the study could be completed on all types of devices). All study materials were translated from German to French by the survey company, and participants were free to choose their preferred language at the onset of the study. An independent back-translation (Brislin, 1970) was performed to identify potential changes in meaning, but only minor adjustments needed to be made. Table S2 provides an overview of the items used for the main predictor and outcome variables (translated into English), and the full study materials (i.e., including original items and back translations) are available from https://osf.io/6t3du.

\section{Informed consent and sociodemographic information}

Participants were first informed about the background and purpose of the study and then provided informed consent. Next, they reported sociodemographic information (i.e., gender, age, canton of residence, zip code, level of education, employment status) and indicated whether they owned a smartphone, as well as the type of device they were using to complete the survey.

\section{General risk perception of $5 \mathrm{G}$}

Next, participants either first rated their general risk perception of $5 \mathrm{G}$ and then proceeded to the next block (see "psychometric paradigm" below), or were assigned to the opposite order (i.e., block-randomized design). Building on Fischhoff and colleagues (Fischhoff et al., 1978), general risk perception was assessed with the question: "In your personal view: How large are the potential risks of 5G in general?" Participants responded to this item (as well as all subsequent items) using a continuous slider that yielded values from 0 to 100 . The endpoints and the midpoint of this slider were labeled "very low", "very high", and "medium".

\section{Psychometric paradigm}

The block implementing the psychometric paradigm comprised 10 items (presented in a randomized order), which were primarily selected based on Slovic and colleagues (table 8 of study 3; Slovic et al., 1985), and 
reflected the 5 items loading most strongly on "factor 1" (dread) and the 5 items loading most strongly on "factor 2" (unknown risk). Most of these items were also part of the 9 dimensions originally proposed and implemented by Fischhoff and colleagues (Fischhoff et al., 1978). To illustrate, an item representing factor 1 (dread) was "How easily can the potential risks of $5 \mathrm{G}$ be reduced?", and an item representing factor 2 (unknown risk) was "To what extent are the potential risks of 5G known to science?" See table S2 for the wording of all items.

\section{Attention check}

The previous block also included an attention-check question, prompting participants to move the slider entirely to the left. As specified in the preregistration, the data of participants who failed to do so (i.e., a value higher than 10, thus permitting a small margin of error) were excluded from the analysis.

\section{Perception of health risks}

The next block tapped participants' perceptions of health risks (i.e., arguably the strongest concern related to $5 \mathrm{G}$ in the general public). To be able to compare the degree of $5 \mathrm{G}$ 's perceived health risks with that of other potential health hazards, participants rated their perception of health risks concerning 8 additional technologies and activities (e.g., $3 \mathrm{G} / 4 \mathrm{G}$, the current cellular network technology; smoking; vaccination). To render comparative judgments possible, the 9 items were presented simultaneously and in a randomized order. Furthermore, at the end of this block participants had the opportunity to provide any other risks related to 5G (i.e., beyond health risks) that they were potentially concerned about (i.e., free text input). See B.4 for the results of the analysis on perceived health risks.

\section{Perceived benefits, trust, knowledge, policy-related attitudes}

The next block consisted of 14 items (presented in a randomized order) tapping perceived benefits (separately for personal, social and economic benefits; Fischhoff et al., 1978), trust (single item; cf. Siegrist, 1999, 2000), knowledge (two subjective and four objective indicators), and policy-related attitudes (acceptability of potential risks, voting intention, need for more regulation, need for more scientific research). Subjective knowledge was measured with a subjective rating of one's $5 \mathrm{G}$ knowledge and an item capturing the degree of 5 G-related media consumption. Objective knowledge was measured with questions concerning whether the legal regulations are the same or different as compared to $3 \mathrm{G} / 4 \mathrm{G}$ (i.e., current technology), whether radiation limits are the same or different as compared to $3 \mathrm{G} / 4 \mathrm{G}$, and whether the degree of radiation primarily results from the 5G-antennas or the users' devices. Moreover participants rated their confidence that there exists (vs. that there does not yet exist) an active 5G-antenna in their residential municipality or within a boundary of one kilometer (which was verified using a geographic information system analysis; see B.3 for the results of this analysis and participants' knowledge about 5G coverage). See table S2 for the wording of all items.

\section{Person-related characteristics}

A final block tapped participants' general risk preferences (Frey et al., 2017), their openness to new experiences (Costa \& MacCrae, 1992), whether they predominantly see digitalization as an opportunity or as a potential risk, whether they feel to be affected by electromagnetic hypersensitivity, and their political attitude. The latter was optional to report. See table S2 for the wording of all items.

\section{Additional questions and screening for follow-up}

At the end of study 1, participants were prompted to type in a real-life risky situation (unrelated to the research questions of this article and thus not further reported here) and were asked about their willingness to share their address to receive a brief brochure with information concerning $5 \mathrm{G}$ by mail (see experimental conditions implemented in the longitudinal sample of study 2; C.3). 


\section{A.3 Analysis plan}

All analyses were conducted in $R$ version 3.5.3 (R Core Team, 2019), using the packages lavaan (Rosseel, 2012), semPlot (Epskamp \& Stuber, 2017), and BAS (Clyde et al., 2011) for the main analyses. See https://osf.io/6t3du for the full analysis code. The analysis plan of study 1 involved three main steps.

First, psychometric modeling was conducted to implement measurement models for several of the predictor (i.e., potential drivers of risk perception) and outcome variables (i.e,. perceived risk and benefit, policy-related attitudes), in order to obtain (as reliable as possible) latent variables of the constructs to be measured (see B.1).

Second, for validation reasons the independent measurement models were linked by means of structural equation modeling, to thus examine the associations between particular drivers and outcome variables as to be expected based on previous theory (see B.2).

Third and finally, the main analysis of study 1 was conducted, namely, a Bayesian model comparison testing the predictive power of the various drivers for perceived risk, perceived benefit, and policy-related attitudes. The latent variables extracted from the various measurement models were used as input to this analysis. Multi-model inference analyses were conducted for two reasons: On the one hand, traditional regression analyses typically involve a two-step approach, aimed at first identifying a single-best model, to then interpret this model's parameters for inference. Yet, the uncertainty from the first step (i.e., identification of best model) is ignored in the second step, resulting in overconfident parameter estimates and poor generalizability (van den Bergh et al., 2020). By contrast, model-averaged coefficients (i.e., parameter estimates that are averaged across the entire model space, weighted by the models' posterior probabilities) avoid this problem and provide more reliable estimates (Hinne et al., 2019; van den Bergh et al., 2020). On the other hand, multi-model inference permits quantifying the contributions of each predictor variable to predicting the outcome variables across the entire model space (e.g., posterior inclusion probabilities), as well as to conduct specific comparisons (e.g., relative evidence of the best model over the model that in- / excluded one of the predictors; this is the analysis reported in Fig. 2 of the main text and in figs. S10 and S12). Multi-model inference is thus a particularly promising approach in scenarios such as the current one, where various predictors (i.e., psychological drivers of risk perception) may not be entirely independent from each other; specifically, the uncertainty concerning the predictors' contributions to predicting the outcome variables will be reflected in the posterior model probabilities, and will accordingly be taken into account in the model-averaged estimates. The analyses were conducted using the R-package $B A S$ (Clyde et al., 2011), implementing linear regression models with Gaussian distributions and identity link functions.

\section{A.4 Deviations from preregistered analysis plan}

The analyses deviated in three respects from the preregistered analysis plan. First, the sample was not split into separate exploratory and confirmatory subsamples, because the measurement models were implemented as preregistered (except one minor modification; see section B.1), thus only using confirmatory factor analyses. Second, the R-package BAS (Clyde et al., 2011) was used instead of the BayesFactor package, because the former renders possible to adaptively screen a large number of different model specifications, as well as to extract model-averaged coefficients. Third and relatedly, no specification curve analysis (SCA) was conducted because the $B A S$ package directly renders possible a multiverse analysis, thus making an additional SCA somewhat redundant.

\section{B Extended results study 1}

\section{B.1 Measurement models}

The measurement models were preregistered on https://osf.io/6t3du and the respective items are described in detail in section A.2; see Fig. S3 for an overview and Tab. S2 for the wording. Prior to implementing the various measurement models, Pearson correlations were compared with Spearman correlations across all 
indicators; as the mean (maximum) absolute difference between the two types of correlations was only .008 (.062), all subsequent analyses were conducted based on the former. The fit of the measurement models with more than one indicator was evaluated according to the following criteria: comparative fit index (CFI), Tucker-Lewis index (TLI), root mean square error of approximation (RMSEA), proportion of explained variance $\left(R^{2}\right)$.

Psychological drivers. The two dimensions of the psychometric paradigm were implemented with two measurement models, one for dread risk (DREAD; A in Fig. S3) and one for unknown risk (UNKNO; B in Fig. S3). Both measurement models included five indicators (adapted from the original psychometric paradigm) and achieved satisfactory fits (DREAD: CFI $=.98, \mathrm{TLI}=.96, \mathrm{RMSEA}=.04, R^{2}=.26$; UNKNO: CFI $=$ .9 , TLI $=.81$, RMSEA $=.08, R^{2}=.22$ ). Trust (C in Fig. S3) was implemented with a single indicator (in line with previous research; e.g., Siegrist et al., 2007), namely, the degree of trust in the authorities responsible for regulating $5 \mathrm{G}$. Note that the downside of a single-indicator model may consist of a relatively lower measurement reliability (i.e., depending on the degree of measurement error of the indicator variable), thus potentially resulting in attenuated associations with other variables of interest. For knowledge, it turned out that the two items measuring subjective knowledge (i.e., a subjective rating of one's $5 \mathrm{G}$ knowledge; an item capturing the degree of 5G-related media consumption) correlated only weakly with the four items measuring objective knowledge (i.e., knowledge concerning the main sources of radiation, frequencies in use, legal radiation limits, and current network coverage; see section B.3). Accordingly, a measurement model with two separate factors (KNsubj and KNobj; D in Fig. S3) was implemented, and this solution achieved a satisfactory fit $\left(\mathrm{CFI}=.97\right.$, TLI $\left.=.94, \mathrm{RMSEA}=.03, R^{2}=.33\right)$. This implementation was the only deviance from the preregistered models, and thus no separate exploratory and confirmatory subsamples were used in study 1 (as originally specified in the preregistration, in case there would have been a need for substantial exploratory analyses; note that this model was subsequently also cross-validated in the independent crosssectional sample of study 2; see D.1). The measurement model for a person's general preference for progression (PROGR; E in Fig. S3) included three indicators, namely, a person's risk preference, openness to new experiences, and attitude towards digitalization. This model also achieved a satisfactory fit (CFI $=1$, TLI $=1, \operatorname{RMSEA}=0, R^{2}=.32$ ).

Perceived risk and benefit. Perceived risk (PRISK; F in Fig. S3) was composed of a single indicator (in line with previous research; Fischhoff et al., 1978), namely, participants' rating of their general risk perception of 5G. Perceived benefit (PBENE; G in Fig. S3) summarized the indicators for perceived personal benefit, benefit for society, and benefit for the economy; these three variables correlated highly between each other and resulted in a satisfactory fit of the respective measurement model $\left(\mathrm{CFI}=1, \mathrm{TLI}=1, \mathrm{RMSEA}=0, R^{2}\right.$ $=.61)$.

Policy-related attitudes. Finally, policy-related attitudes (POLICY; H in Fig. S3) was implemented with one measurement model and involved the following indicators: acceptability of risk, voting intention, need for more regulation, and need for more research. This measurement model achieved a satisfactory fit $\left(\mathrm{CFI}=.99, \mathrm{TLI}=.98, \mathrm{RMSEA}=.08, R^{2}=.62\right)$.

The factor scores extracted from the various measurement models (see Fig. S4 for their distributions and Fig. S5 for their inter-correlations) were standardized and constituted the input for the subsequent Bayesian model comparison analyses. To be able to depict the associations between the various predictor and outcome variables on the original response scale (i.e., Fig. 2 and 4 of the main text), as well as to be able to compute intraclass-coefficients using the original response scale, approximations for the factor values were computed. Specifically, for each latent variable with multiple indicators, the latter were unit-weighted and averaged. The resulting scores correlated very highly with the original factor scores, namely, $r=.94$ (dread), .96 (unknown risk), .86 (objective knowledge), .85 (subjective knowledge), and .89 (preference for progression).

\section{B.2 Structural equation models}

Prior to comparing the various potential drivers of risk perception directly with each other (i.e., the main analysis of study 1), four separate structural equation models (SEMs; A-D in Fig. S6) were implemented. 
These SEMs linked the various measurement models and thus permitted validating the measurement models and testing a number of associations predicted by previous theory. The four SEMs were preregistered on https://osf.io/6t3du.

First, according to the psychometric paradigm (A in Fig. S6) dread risk and unknown risk drive the perception of risks (positively) and benefits (negatively), and as a consequence the latter two dimensions may be inversely related. These patterns have repeatedly been observed empirically and in diverse contexts (Alhakami \& Slovic, 1994; Finucane et al., 2000; Fischhoff et al., 1978; Weber et al., 2002). The implementation of the psychometric paradigm at the level of individual participants performed very well, with a CFI $=.94$, a $\mathrm{TLI}=.92$, and a RMSEA $=.06$. Moreover, dread risk was, as predicted, strongly and positively associated with perceived risk, as well as a strongly but negatively associated with perceived benefit. However, the predicted associations of unknown risk did not emerge.

Second, according to the trust-knowledge model (B in Fig. S6), trust and knowledge should both be negatively related to perceived risk but positively related to perceived benefit. The implementation of this model, with separate factors for subjective and objective knowledge (KNsubj and KNobj, respectively; see section B.1), also resulted in a satisfactory fit, namely, a CFI $=.97$, a TLI $=.95$, and a RMSEA $=.05$. Moreover, the model confirmed that trust was strongly and negatively associated with perceived risk, as well as strongly but positively associated with perceived benefit - in line with the predictions. Furthermore, objective knowledge (but not subjective knowledge) was negatively associated with perceived risk, and both were weakly and positively associated with perceived benefit.

Third, a person's general preference for progression (C in Fig. S6), that is, a potentially quite stable person-specific driver, was expected to be negatively associated with perceived risk and to be positively associated with perceived benefit. This model also performed very well, with a CFI $=.97$, a TLI $=.95$, and a RMSEA $=.07$. In line with the predictions, a person's general preference for progression was negatively associated with perceived risk, as well as strongly and positively associated with perceived benefit.

Fourth and finally, perceived risk and benefit were tested concerning their role in shaping policy-related attitudes, with an expected negative influence of perceived risk and a positive influence of perceived benefit (D in Fig. S6). The model performed very well, with a CFI $=.99$, a TLI $=.99$, and a RMSEA $=.05$. Moreover, as expected perceived risk was strongly and negatively associated with policy-related attitudes, whereas perceived benefit proved to be strongly and positively associated with policy-related attitudes.

In sum, all four SEMs achieved satisfactory fits, and most of the estimated paths were strongly in line with the associations to be expected based on previous theory. As these models are non-nested, a direct comparison of the psychological drivers' predictive power is not straightforward, which is why the extracted factor scores were used to conduct an extensive Bayesian model comparison (i.e., the main analysis of study $1)$.

\section{B.3 GIS analysis of network coverage}

Based on publicly available and up to date information on 5G-antenna locations in Switzerland (retrieved from https://data.geo.admin.ch/ch.bakom.mobil-antennenstandorte- $5 \mathrm{~g} /$ ), a geographic information system (GIS) analysis was conducted to determine whether an active 5G-antenna existed in each residential municipality (or within a boundary of one kilometer thereof) - rendering possible to evaluate participants' respective ratings objectively using the reported zip codes.

This analysis revealed that overall participants' knowledge concerning 5G coverage was very limited. Specifically, as can be seen in Figure S7 (first panel) many participants were very unsure about whether 5G coverage existed in their municipality at the time of the study (i.e., many ratings around the mid-point of the scale, indicating maximal uncertainty). Moreover, among participants with actual 5G coverage (second panel), about equally many were convinced that coverage existed as were convinced that coverage did not yet exist (ratings at the extremes of the scale). Among participants without 5G coverage, relatively more participants (correctly) believed that that coverage did not yet exist, whereas only a minority (incorrectly) believed that coverage already existed. 


\section{B.4 Perception of health risks}

To specifically gauge participants' perceptions of health risks associated with 5G (particularly compared to $4 \mathrm{G}$, the current mobile network technology in Switzerland), a comparison of nine potential health hazards was conducted (Fig. S8). According to this analysis, participants perceived 5G to constitute a credibly higher health risk $(M=60.3)$ as opposed to $4 \mathrm{G}\left(M=47.9 ; M_{\Delta}=12.4,95 \%\right.$ highest-density interval [HDI] $=11.1-$ 13.7); despite that in Switzerland the same legal radiation limits apply, and virtually the same frequencies are currently used. Furthermore, on average $5 \mathrm{G}$ was perceived to constitute a substantially higher health risk as compared to vaccinations.

\section{Detailed methods study 2}

Study 2 was identical with study 1, except for four additional questions related to the experimental manipulation (in the longitudinal sample) that was administered in between the two studies (see C.2).

\section{C.1 Participants and sample characteristics}

Study 2 was conducted in January 2020 and involved two separate samples: for the cross-sectional sample, it was aspired to newly recruit 1,000 participants, resulting in a final sample size of $N=1,013$ participants after applying the same criteria as described for study 1. For the longitudinal sample, the goal was to collect retest data for 1,000 participants of study 1 (i.e., 250 participants for each of the four experimental conditions, see C.3). Assuming an attrition rate of about 30\%, 1,400 participants were thus sampled from study 1 (i.e., from all participants who indicated willingness to receive a letter with information on $5 \mathrm{G}$ by mail; $69.5 \%$ of participants). After applying the same criteria as described for study 1, and after removing 130 participants from the treatment groups who were not aware that they had received a letter with information about $5 \mathrm{G}$ from the survey company (i.e., the intervention of the field experiment) a final sample size of $N=839$ participants resulted. Table S1 reports sociodemographic information. Participants received the same compensation as in study 1, and participants in the longitudinal sample received a bonus of 100 points as an additional incentive to also complete study 2 .

\section{C.2 Procedure and materials}

The procedures and materials were identical to those of study 1 , with some additional questions at the end of study 2. Specifically, all participants were asked about their awareness of the published expert report, and in case of an affirmative response, they reported (a) how extensively they had studied the full report or a summary thereof, (b) how well they felt informed by the expert report, and (c) to what extent they believed that their attitudes towards $5 \mathrm{G}$ had changed due to the expert report (Fig. S13). Moreover, as a manipulation check participants in the longitudinal sample were explicitly asked whether they had recently received a letter containing information on $5 \mathrm{G}$ from the survey company.

\section{C.3 Expert report on 5G and experimental conditions in the longitudinal sam- ple}

On November 28, 2019, the Swiss Federal Office for the Environment (FOEN) publicly released an expert report on 5G. This report was developed in an interdisciplinary working group on behalf of the Federal Department for the Environment, Transport, Energy and Communication. The group included representatives of federal offices and cantonal authorities, mobile phone providers, doctors, and representatives of cities and municipalities. Its task was to analyze the needs and risks involved in setting up 5G networks and to develop recommendations. It explains technical facts about $5 \mathrm{G}$, deals with the operation of the Swiss 
mobile radio networks and their regulation, estimates the exposure of the population to non-ionizing radiation, and summarizes the scientific findings on possible health consequences. The report is available from https://www.bafu.admin.ch/5g, including a management summary in English that can be retrieved from https://www.newsd.admin.ch/newsd/message/attachments/59387.pdf. Note that the report sketched different scenarios of how the Swiss mobile radio network could be further developed. However, the working group did not make a specific recommendation concerning which option is best. Rather, the report was meant to serve as an evidence base for informing the general public and for policy making in the Swiss government.

\section{Experimental conditions (longitudinal sample)}

Participants of study 1 who were sampled for the longitudinal track were randomly assigned to one of four between-subjects conditions: There were three treatment conditions (groups $\mathrm{A}-\mathrm{C}$ ) and a control condition (group D). In between the two studies and about one week prior to the data collection of study 2, participants in groups $\mathrm{A}-\mathrm{C}$ received a letter with information concerning the expert report on $5 \mathrm{G}$, which was released by the FOEN on November 28, 2019 (i.e., shortly after the data collection of study 1). Specifically:

- Participants of group A received the official management summary of the report (8 pages long; for the English version, see https://www.newsd.admin.ch/newsd/message/attachments/59387.pdf).

- Participants of group B received the official press release for the report (2 pages long; only available in German and French, see https://www.bafu.admin.ch/bafu/de/home/themen/elektrosmog/mitteilungen. msg-id-77294.html).

- Participants of group C received a list of four key points (1 page long) that were extracted from the management summary. Three of these points summarized the legal situation concerning radiation limits in Switzerland, such as concerning general immission limits (i.e., the same as in the majority of neighboring countries), installation limits, and regulations at places with sensitive use (e.g., schools, hospitals) - and that these regulations thus result in stricter overall limits for non-ionising radiation from mobile communications antennas compared to most European countries. The fourth point summarized technical aspects of $5 \mathrm{G}$, such as that the frequencies currently available for $5 \mathrm{G}$ in Switzerland are comparable with those of $4 \mathrm{G}$ and WLAN. These elements were selected because they represent facts all members of the expert group agreed on, and because they may have an impact on people's trust, experienced dread, or knowledge - which turned out to be important psychological drivers of people's risk perceptions according to the analysis of study 1.

- Participants of group D served as controls and did not receive any information by mail.

The motivation for using these naturalistic information formats were twofold: First, to fully adhere to the publicly available evidence base and to thus test the potential effects of realistic interventions. Second, to maximize the chance of inducing intraindividual change in any of the psychological drivers (e.g., dread, trust, knowledge), as the most concise and accessible format (the 1-page with key points) may boost, for instance, trust and knowledge for some persons, whereas for others a more extensive information format (the detailed 8-page summary) may be required to this end, with the short format possibly even triggering the opposite effect.

\section{C.4 Analysis plan}

In study 2 , the analysis plan involved multiple steps for each of the two independent samples.

\section{Cross-sectional sample: Cross-validations and mean-level change in the population}

Using the new (i.e., cross-sectional) sample of participants in study 2, the implementation of the measurement models and the analyses of the drivers of interindividual differences (as conducted in study 1) were first replicated as a robustness check, in order to cross-validate these results (see D.1). Second, the cross-sectional sample was also used to gauge whether any mean-level changes in perceived risk and benefit, as well as in policy-related attitudes, occurred in the population - that is, during the time period between study 1 and study 2 (e.g., due to the public release of the national expert report and associated media coverage; see D.2). 


\section{Longitudinal sample: Field experiment and intraindividual change}

In the longitudinal sample, the degree of intraindividual change in the various drivers of risk perceptions was first assessed (see the main text for the change in the hazard-related drivers, and D.3 for the change in person-specific drivers). Next, Bayesian regression analyses were conducted to examine whether any of the experimental conditions triggered systematic mean-level differences in i) the potential drivers of risk perception, and ii) the various outcome variables. Finally, a Bayesian model comparison analogous to that of study 1 - yet based on the $\Delta$ 's of the predictor and outcome variables - was conducted to examine whether intraindividual change in any of the psychological drivers systematically related to intraindividual change in the outcome variables (see D.4).

\section{Extended results study 2}

\section{D.1 Cross-validations of study 1 (cross-sectional sample)}

Before turning to the main research question of the second study, the data of the newly recruited crosssectional sample was used for two robustness checks. First, the same measurement models as in study 1 were implemented for the various predictor and outcome variables. As Figure S9 shows, the respective model fits and estimated paths were highly congruent with those observed in study 1 (see Fig. S3) - thus corroborating the robustness of these measurement models.

Second, the same Bayesian model comparison as in the main analysis of study 1 was implemented. The results are depicted in Figure S10 and are closely in line with those of study 1 (Fig. 3 of the main text). The only notable exceptions included that gender differences were less pronounced for perceived risk and policy-related attitudes, and there was no longer an effect of education on perceived benefit. Note that the Bayes Factors generally tended to be lower due to the smaller sample size of the cross-sectional sample (i.e., as compared to the sample of study 1).

\section{D.2 Change at the population level (cross-sectional sample)}

Moreover, the cross-sectional sample of study 2 was used to conduct independent-groups comparisons (i.e., between the participants of study 1 and participants of study 2), using the raw ratings of perceived risk, perceived benefit (three separate ratings), and policy-related attitudes (four separate ratings) as dependent variables. These comparisons were conducted by means of Bayesian regression models using rstanarm (Stan Development Team, 2016) and indicated that - at the aggregate level-perceived risk and benefit as well as policy-related attitudes remained virtually unchanged. Specifically, as can be seen in Figure S11, the blue vertical lines in the background depicting the mean differences were all very close to zero. There was only a credible (but small) mean difference across the two studies for perceived benefit for society (i.e., a mean reduction of -2.5 in study $2 ; 95 \% \mathrm{HDI}=-4.4$ to -.6 ). Thus, overall the publication of the expert report did not trigger any substantial mean-level changes in the population during the period between the two studies.

\section{D.3 Intraindividual change in person-specific drivers (longitudinal sample)}

In contrast to hazard-related drivers, person-specific drivers tend to remain relatively stable over time by definition - thus resulting in only modest (or even no) intraindividual change. To verify, intraclass-coefficients (ICCs) were computed for each of the respective continuous variables, and Cohen's kappas were computed for the nominal and ordinal variables. ICCs quantify the degree of intraindividual variance relative to the variance between individuals: An ICC of 1 represents no intraindividual variability, an ICC of 0 represents pure intra- and no interindividual variability, and an ICC of .5 represent the same degree of variability between individuals as within individuals. Conversely, Cohen's kappa reflects a direct measure of accordance. As to be expected, age had an ICC of 1; gender had a kappa of .98 (thus reflecting a very small degree of response error); occupation had a kappa of .87; political attitude had an ICC of .88, education had a kappa of .78, preference for progression had an ICC of .76; and electromagnetic hypersensitivity (EHS) had an ICC 
of .75 (all values were computed for participants of the control group). Thus, by and large the person-specific drivers remained highly stable over time.

\section{D.4 Associations of intraindividual change in predictor and outcome variables (longitudinal sample)}

The results of this analysis are reported in the main text, see Figure S12 for a graphical depiction. As there was hardly any intraindividual change in the person-specific drivers (as expected; see D.3), these did not account for any intraindividual changes in perceived risk and benefit, nor in policy-related attitudes (Fig. S12). 


\section{E Figures}
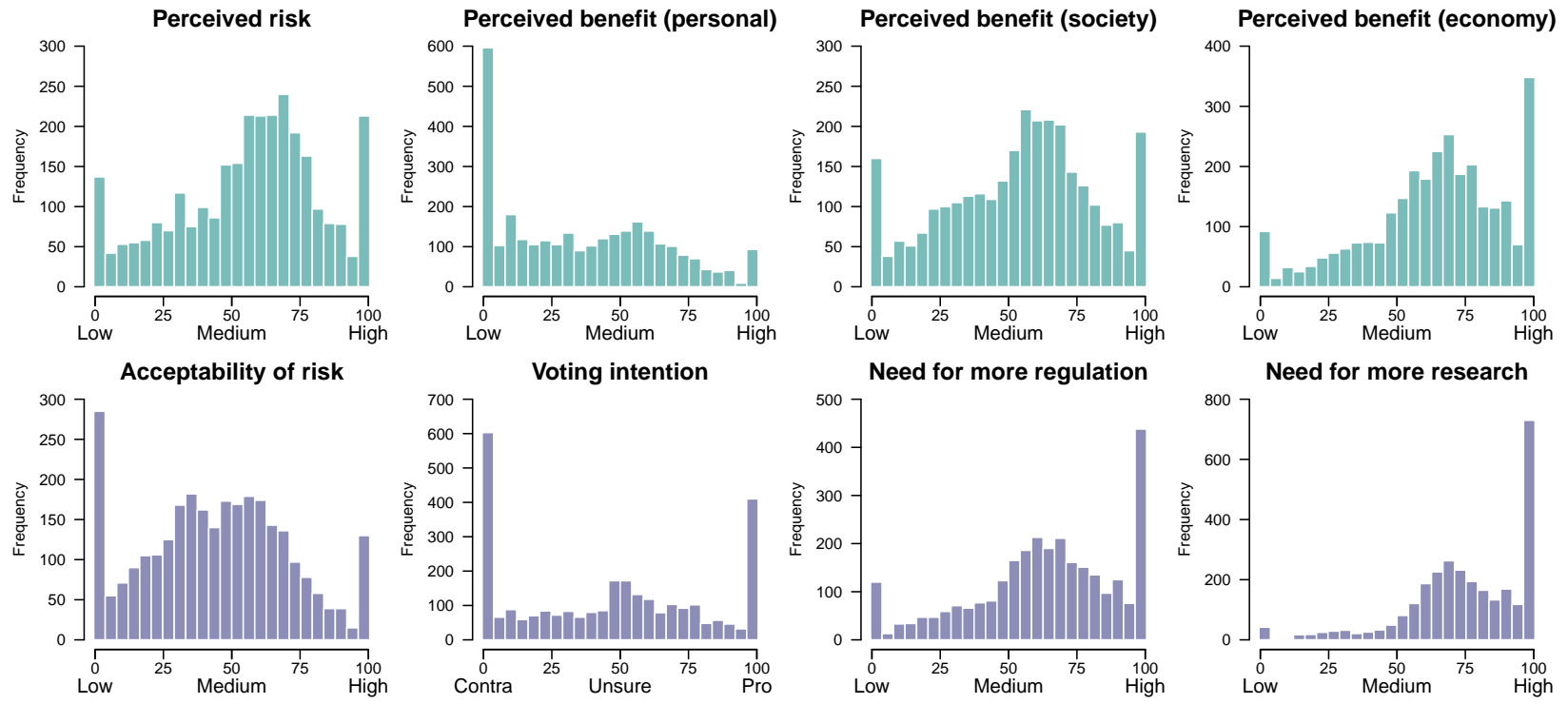

Figure S1: Study $1(\mathrm{~N}=2,919)$ : Distributions of perceived risk and benefit (upper panel) and policy-related attitudes (lower panel). See table S2 for the wording of the items. 


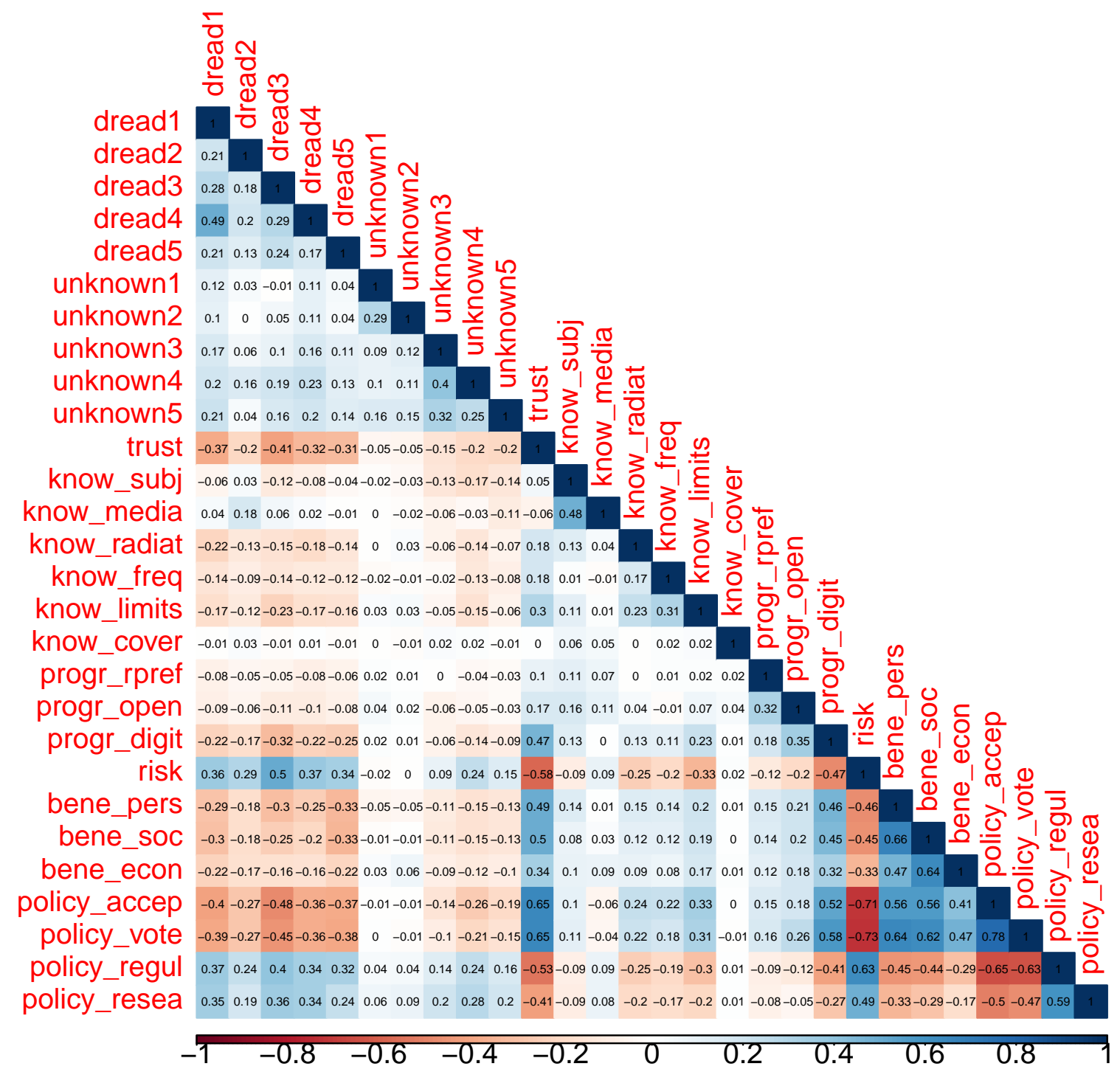

Figure S2: Study $1(\mathrm{~N}=2,919)$ : Correlations between the main variables that were part of the various measurement models (see Fig. S3). See table S2 for the abbreviations and wording of the items. 


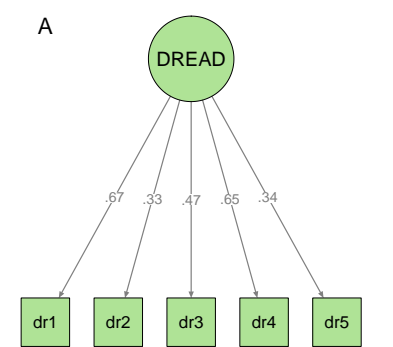

$\mathrm{CFI}=0.98|\mathrm{TLI}=0.96| \mathrm{RMSEA}=0.04 \mid \mathrm{R} 2=0.26$

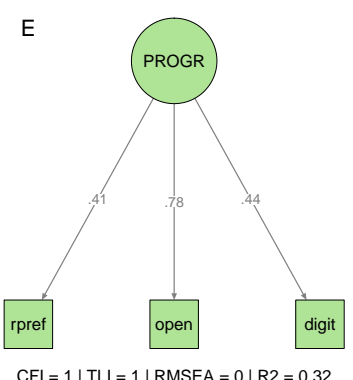

B

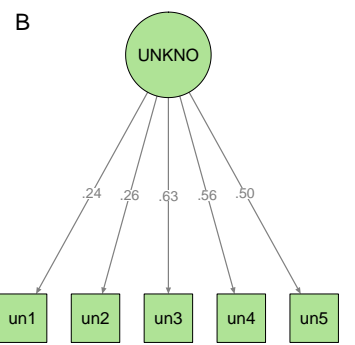

$\mathrm{CFI}=0.9|\mathrm{TLI}=0.81| \mathrm{RMSEA}=0.08 \mid \mathrm{R} 2=0.22$

$\mathrm{F}$

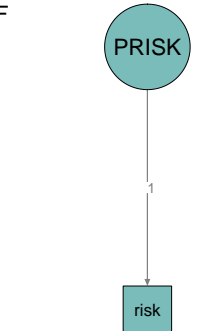

$\mathrm{CFI}=1|\mathrm{TL}|=1|\mathrm{RMSEA}=0| \mathrm{R} 2=1$
C

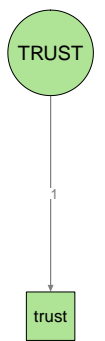

$\mathrm{CFI}=1|\mathrm{TLI}=1| \mathrm{RMSEA}=0 \mid \mathrm{R} 2=1$

$$
\text { G }
$$

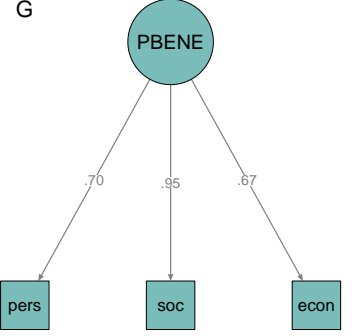

$\mathrm{CFI}=1|\mathrm{TLI}=1| \mathrm{RMSEA}=0 \mid \mathrm{R} 2=0.61$

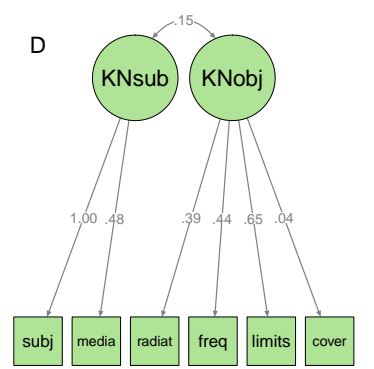

$\mathrm{CFI}=0.97|\mathrm{TLI}=0.94| \mathrm{RMSEA}=0.03 \mid \mathrm{R} 2=0.33$

$\mathrm{H}$

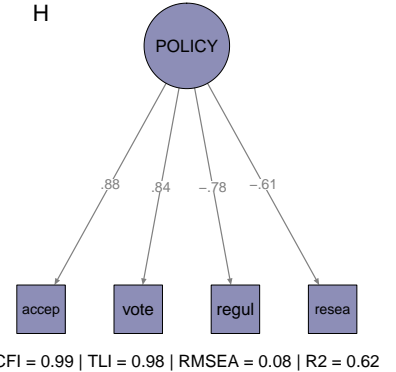

Figure S3: Study $1(\mathrm{~N}=2,919)$ : Measurement models that were implemented using confirmatory factor analyses. The models for the various psychological drivers are depicted in green, and the models for the outcome variables in blue (perceived risk and benefit) and purple (policy-related attitudes). Panel A: DREAD $=$ dread risk. Panel B: UNKNO $=$ unknown risk. Panel C: TRUST $=$ trust. Panel D: KNsubj $=$ knowledge (subjective); KNobj $=$ knowledge (objective). Panel $\mathrm{E}:$ PROGR $=$ preference for progression. Panel $\mathrm{F}$ : PRISK $=$ perceived risk. Panel G: PBENE $=$ perceived benefit. Panel H: POLICY $=$ policy-related attitudes. See section A.2 for a detailed description and table S2 for the abbreviations and wording of the items. 


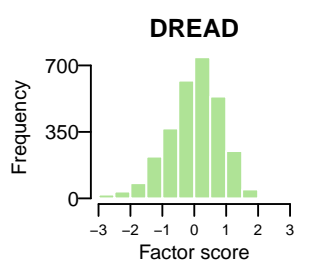

PROGR
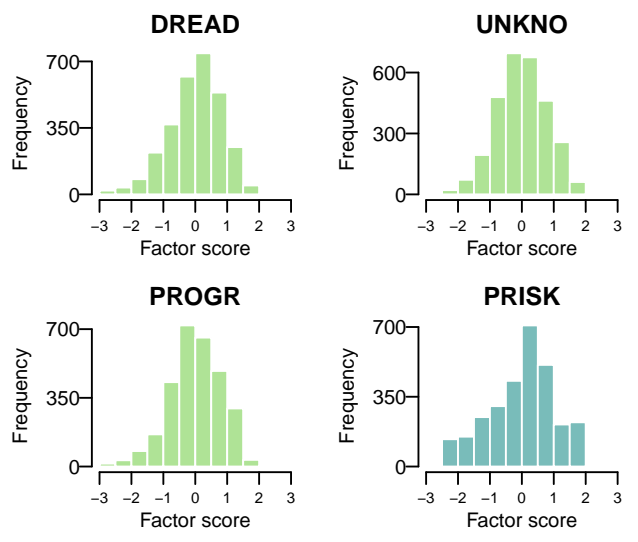

PRISK
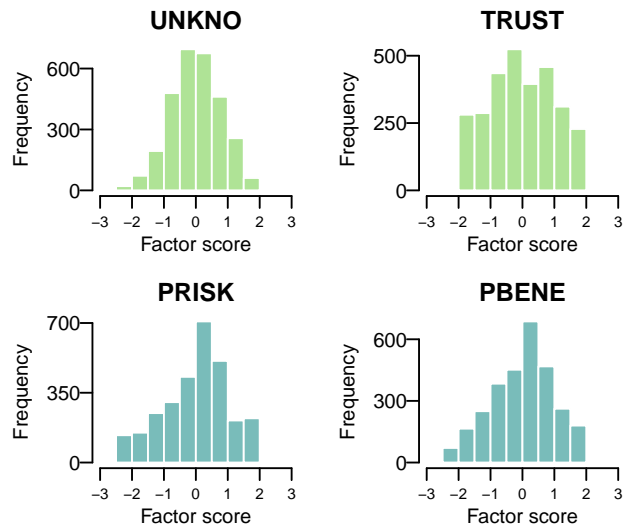

PBENE
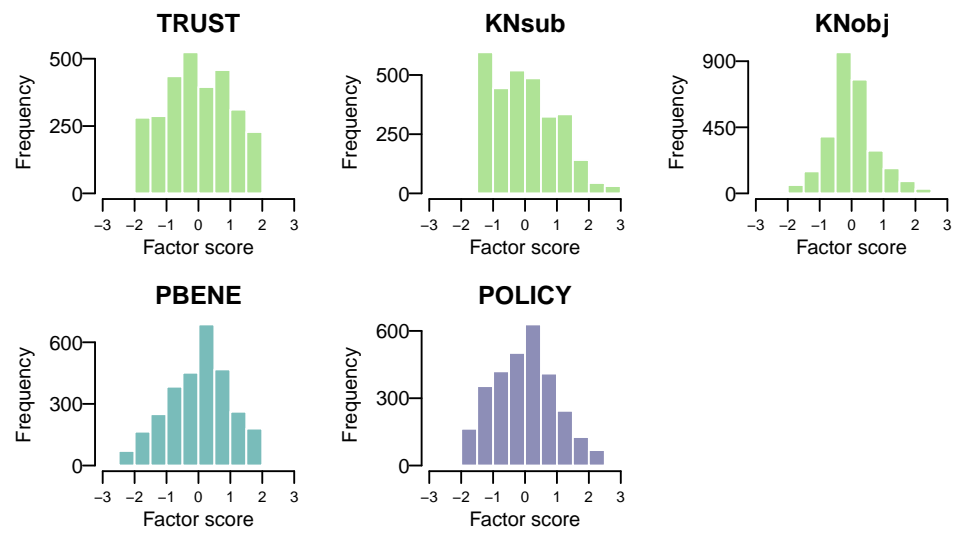

POLICY

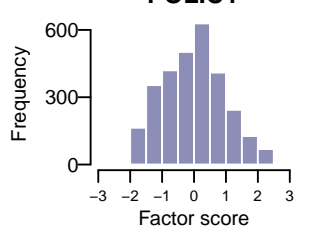

Figure S4: Study $1(\mathrm{~N}=2,919)$ : Distributions of factor scores extracted from the various measurement models (see Fig. S3 for the measurement models and respective abbreviations of the latent variables). 


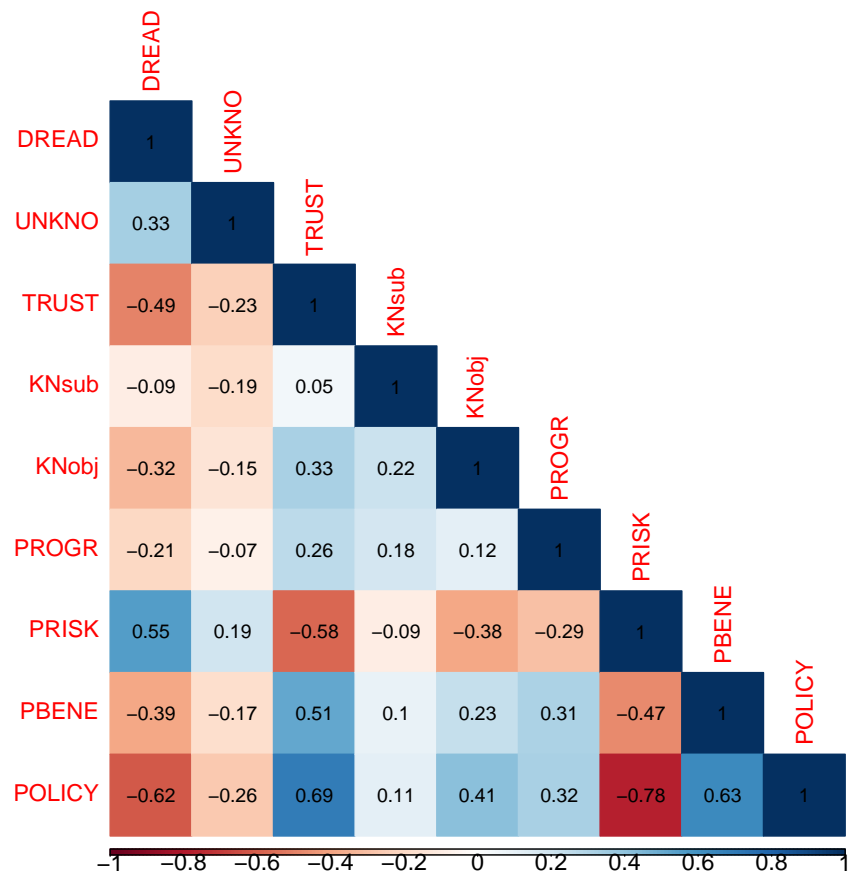

Figure S5: Study $1(\mathrm{~N}=2,919)$ : Correlations between factor scores that were extracted from the various measurement models (see Fig. S3 for the measurement models and respective abbreviations of the latent variables). 
A

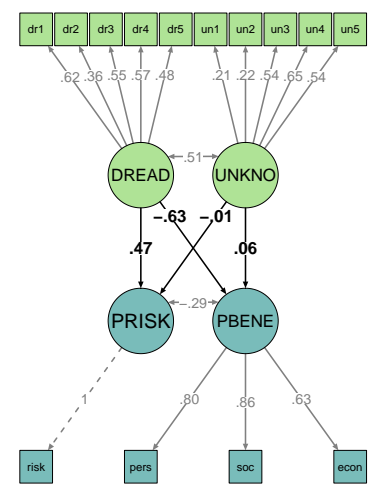

$\mathrm{CFI}=0.94|\mathrm{TLI}=0.92| \mathrm{RMSEA}=0.06$
$\mathrm{B}$

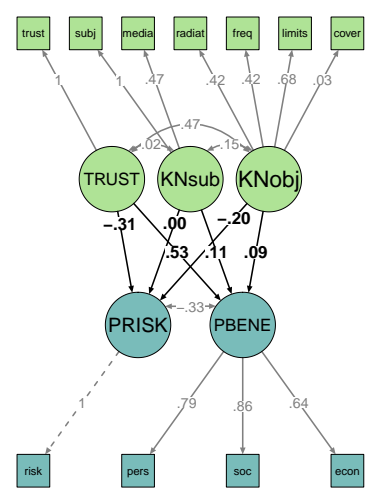

$\mathrm{CFI}=0.97|\mathrm{TLI}=0.95| \mathrm{RMSEA}=0.05$
C

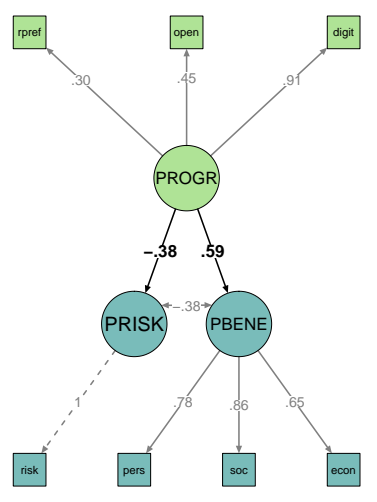

$\mathrm{CFI}=0.97|\mathrm{TLI}=0.95| \mathrm{RMSEA}=0.07$
$\mathrm{D}$

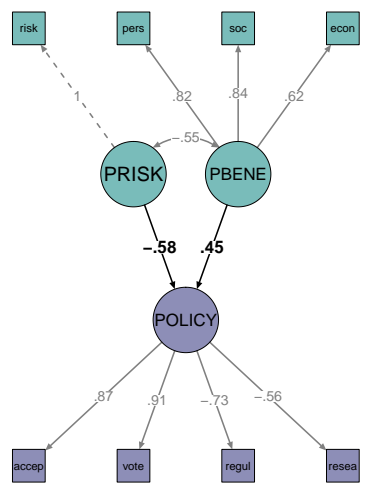

$\mathrm{CFI}=0.99|\mathrm{TLI}=0.99| \mathrm{RMSEA}=0.05$

Figure S6: Study 1 ( $\mathrm{N}=2,919)$ : Four separate structural equation models (SEMs) that linked the various measurement models (see Fig. S3 for the measurement models and table S2 for the abbreviations and wording of the items). These SEMs tested a number of associations to be expected based on previous theory: Panel A depicts the psychometric paradigm involving dread and unknown risk (i.e., hazard-related drivers), panel $\mathrm{B}$ depicts the trust-knowledge model (i.e., hazard-related drivers), panel $\mathrm{C}$ depicts the model testing the role of a person's general preference for progression (i.e., person-specific driver), and panel D depicts the model testing perceived risk and benefit as predictors of policy-related attitudes. 

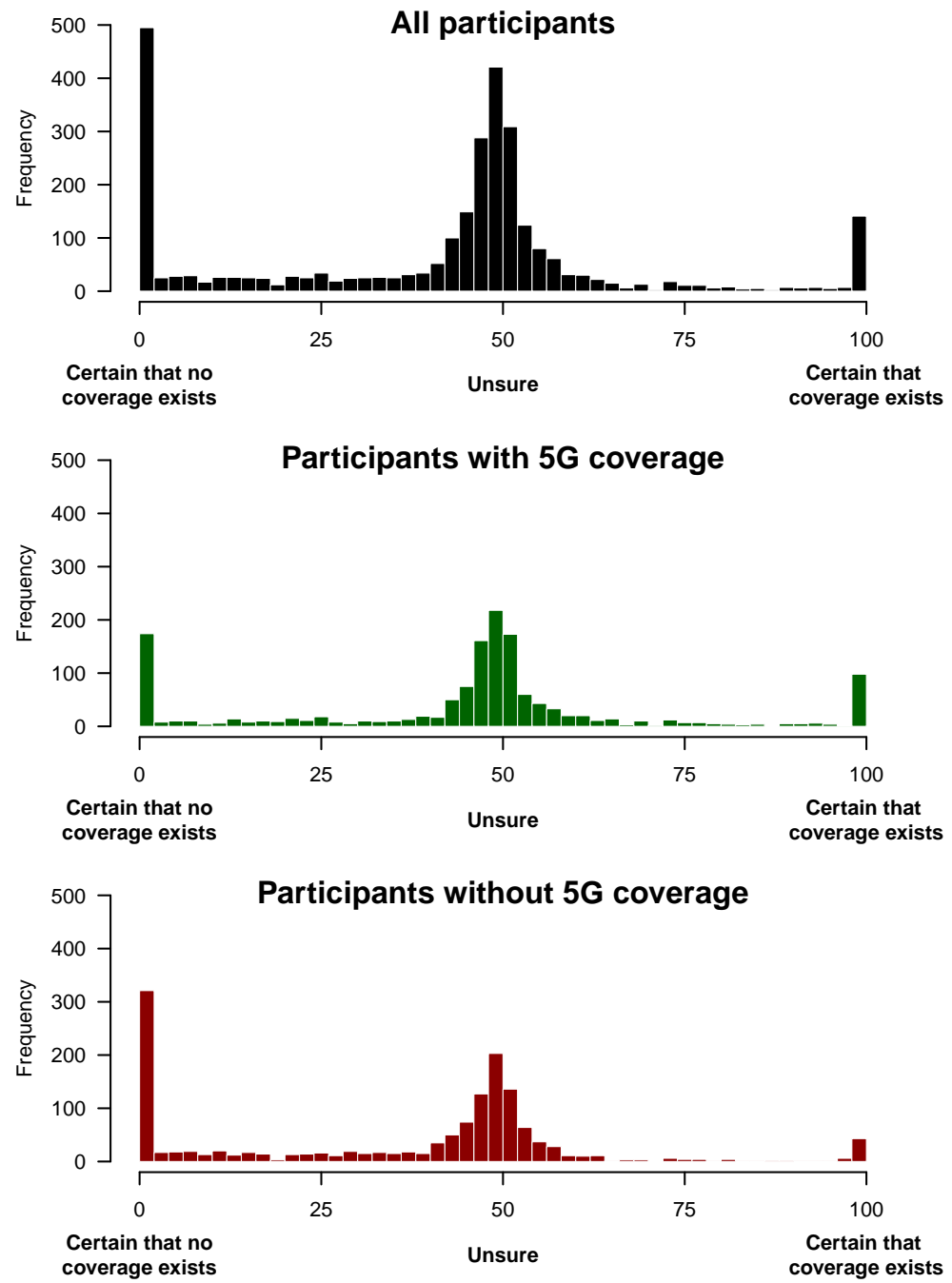

Figure S7: Study $1(\mathrm{~N}=2,919)$ : Participants' objective knowledge about 5G coverage. The ratings express participants' beliefs that $5 \mathrm{G}$ coverage existed at their municipality of residence or within a boundary of 1 kilometer thereof (i.e., at the time of the study). Actual network coverage was determined by means of a GIS-analysis using publicly available data on 5G-antenna locations (see B.3) and participants' zip codes. The panel on the top represents all participants, the panel in the middle represents participants with $5 G$ coverage according to the GIS-analysis, and the bottom panel depicts participants who did not yet have $5 \mathrm{G}$ coverage according to the GIS-analysis. 
Perception of health risks

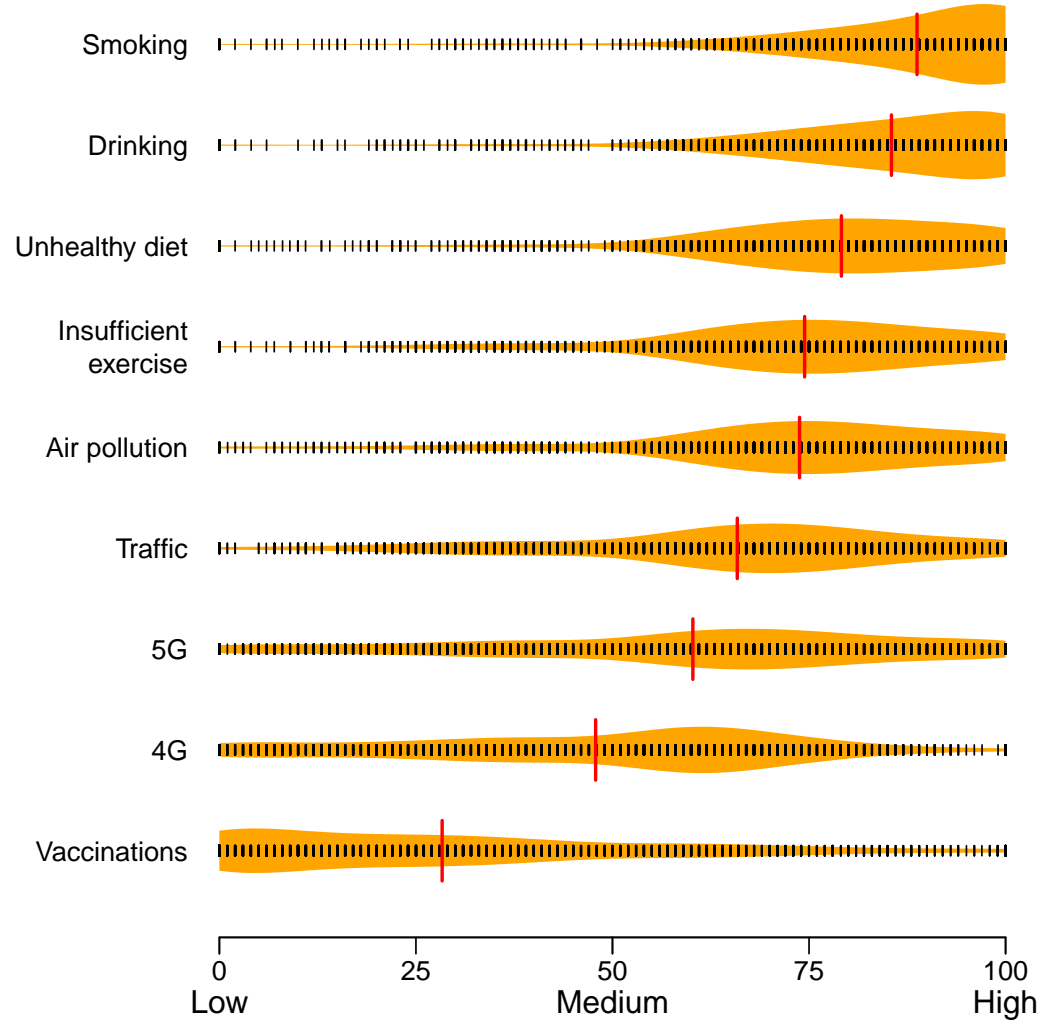

Figure S8: Study $1(\mathrm{~N}=2,919)$ : Comparison of perceived health risks. Each black vertical line represents the rating of one participant (small jitter added), and the red lines depict mean ratings. 


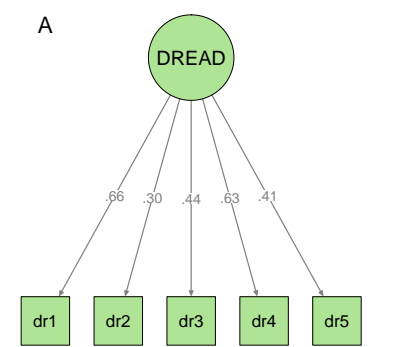

$\mathrm{CFI}=0.98|\mathrm{TL}|=0.97|\mathrm{RMSEA}=0.04| \mathrm{R} 2=0.26$

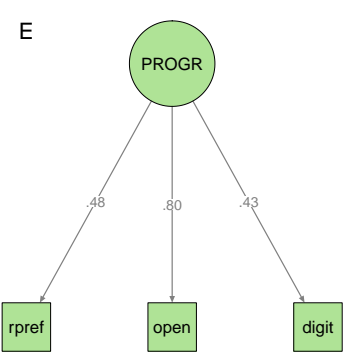

$\mathrm{CFI}=1|\mathrm{TLI}=1| \mathrm{RMSEA}=0 \mid \mathrm{R} 2=0.35$
B

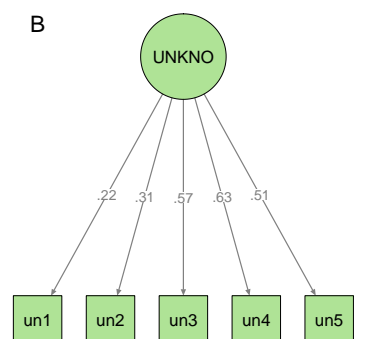

$\mathrm{CFI}=0.87|\mathrm{TLI}=0.74| \mathrm{RMSEA}=0.1 \mid \mathrm{R} 2=0.22$

$\mathrm{F}$

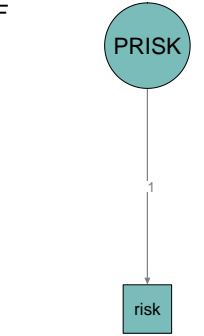

$\mathrm{CFI}=1|\mathrm{TLI}=1| \mathrm{RMSEA}=0 \mid \mathrm{R} 2=1$
C

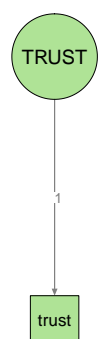

$\mathrm{CFI}=1|\mathrm{TL}|=1|\mathrm{RMSEA}=0| \mathrm{R} 2=1$

$$
\text { G }
$$

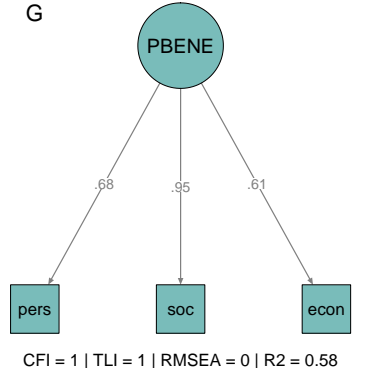

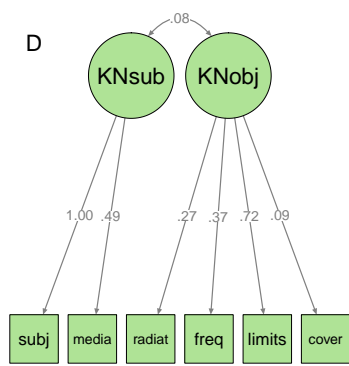

$\mathrm{CFI}=0.86|\mathrm{TLI}=0.77| \mathrm{RMSEA}=0.07 \mid \mathrm{R} 2=0.33$

$\mathrm{H}$

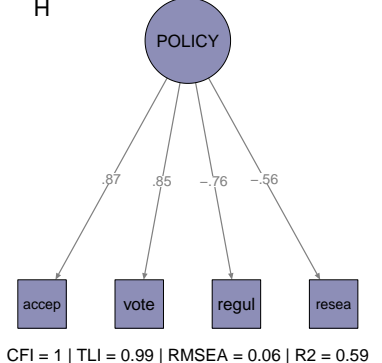

Figure S9: Study 2 (cross-sectional sample, $N=1,013)$ : Measurement models that were implemented as a robustness check of study 1 . Panel A: DREAD = dread risk. Panel B: UNKNO = unknown risk. Panel C: TRUST $=$ trust. Panel D: KNsubj $=$ knowledge (subjective) KNobj $=$ knowledge (objective). Panel $\mathrm{E}: \mathrm{PROGR}=$ preference for progression. Panel F: PRISK $=$ perceived risk. Panel G: PBENE = perceived benefit. Panel H: POLICY = policy-related attitudes. See Figure S3 for the equivalent models originally implemented in study 1, and table S2 for the abbreviations and wording of the items. 


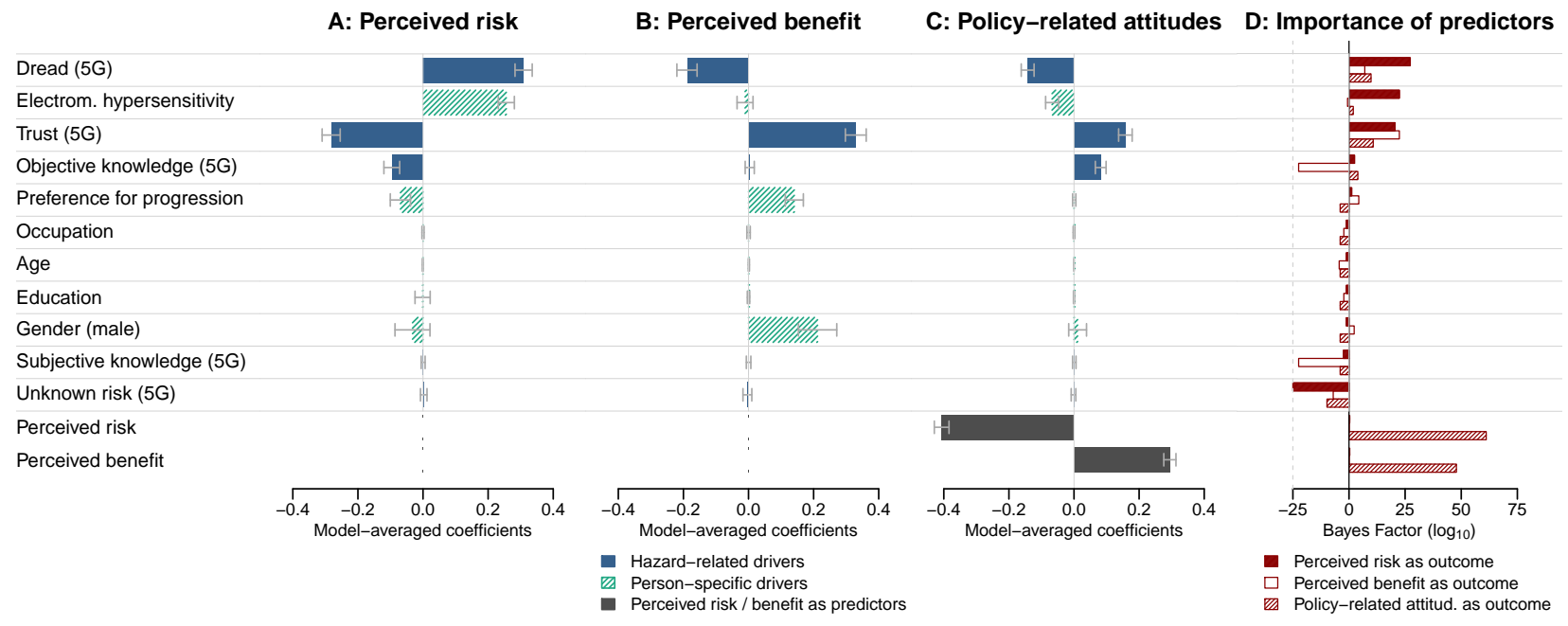

Figure S10: Study 2 (cross-sectional sample, $\mathrm{N}=1,009$ after four list-wise deletions due to missing values): Results of the Bayesian model comparison that was implemented in the independent (i.e., cross-sectional) sample of study 2 as a robustness check of study 1 (see Fig. 3 in the main text). Panel A depicts the coefficients for perceived risk as outcome variable, panel B depicts the coefficients for perceived benefit as outcome variable, and panel $\mathrm{C}$ depicts the coefficients for policy-related attitudes as outcome variable. Panel D depicts the Bayes Factors of all predictor variables, separately for the three outcome variables. 

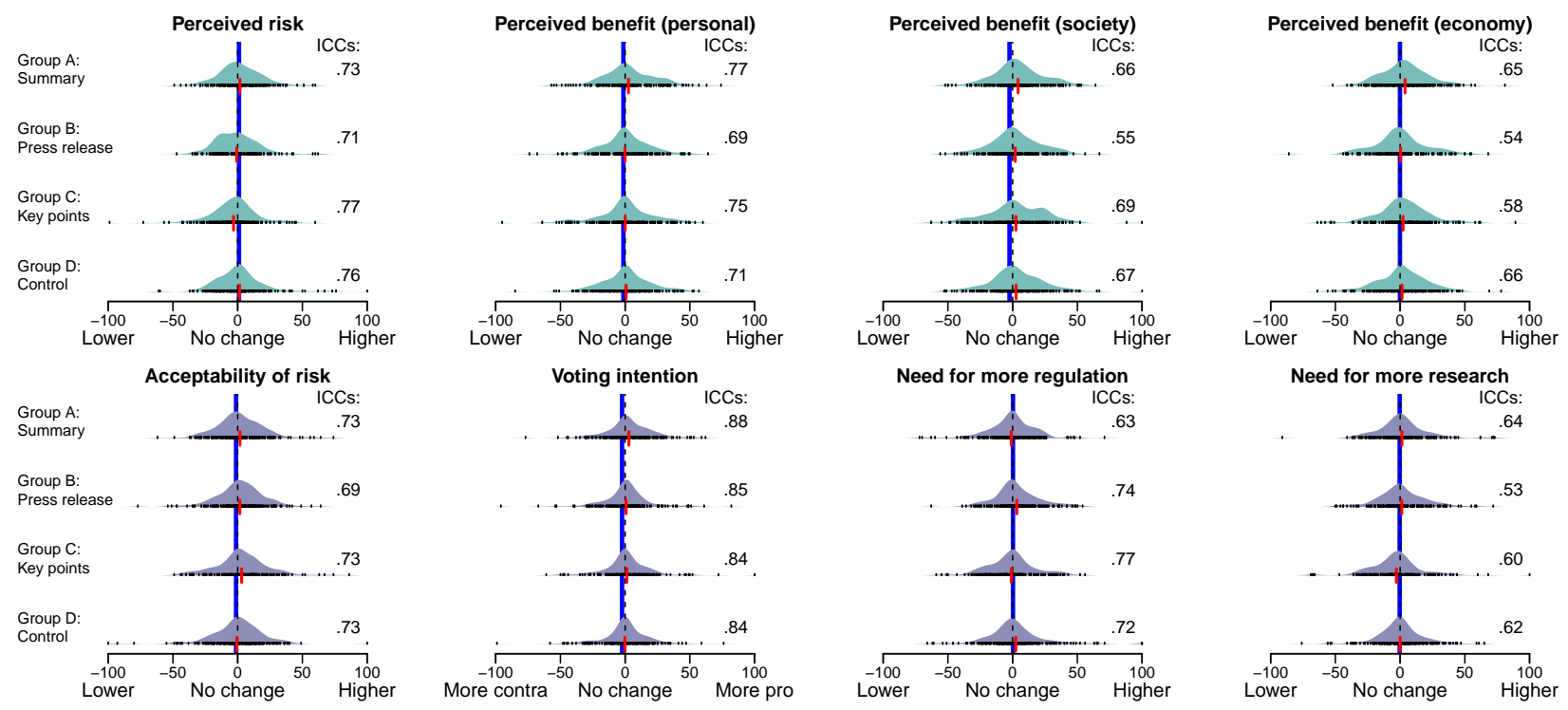

Figure S11: Distributions of intraindiviudal change (across the two studies) in the main outcome variables, separately for the four experimental conditions. The red vertical lines depict average changes per variable and experimental condition (longitudinal sample, $\mathrm{N}=839$ ). The blue vertical lines in the background depict the mean-level change from study 1 to study 2 in the population (i.e., cross-sectional sample, $\mathrm{N}=1,013$ ). 


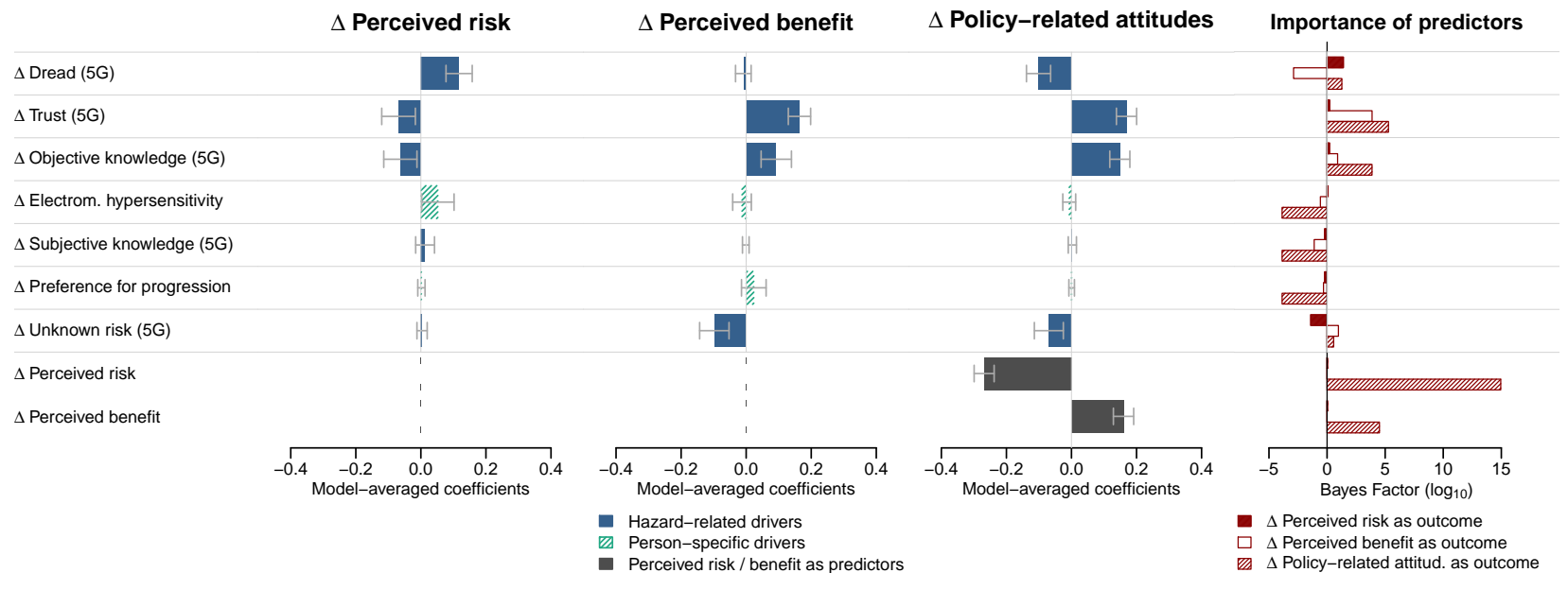

Figure S12: Study 2 (longitudinal sample, $\mathrm{N}=839$ ): Results of the Bayesian model comparison based on intraindividual changes (i.e., $\Delta$ ) in the predictor and outcome variables. 

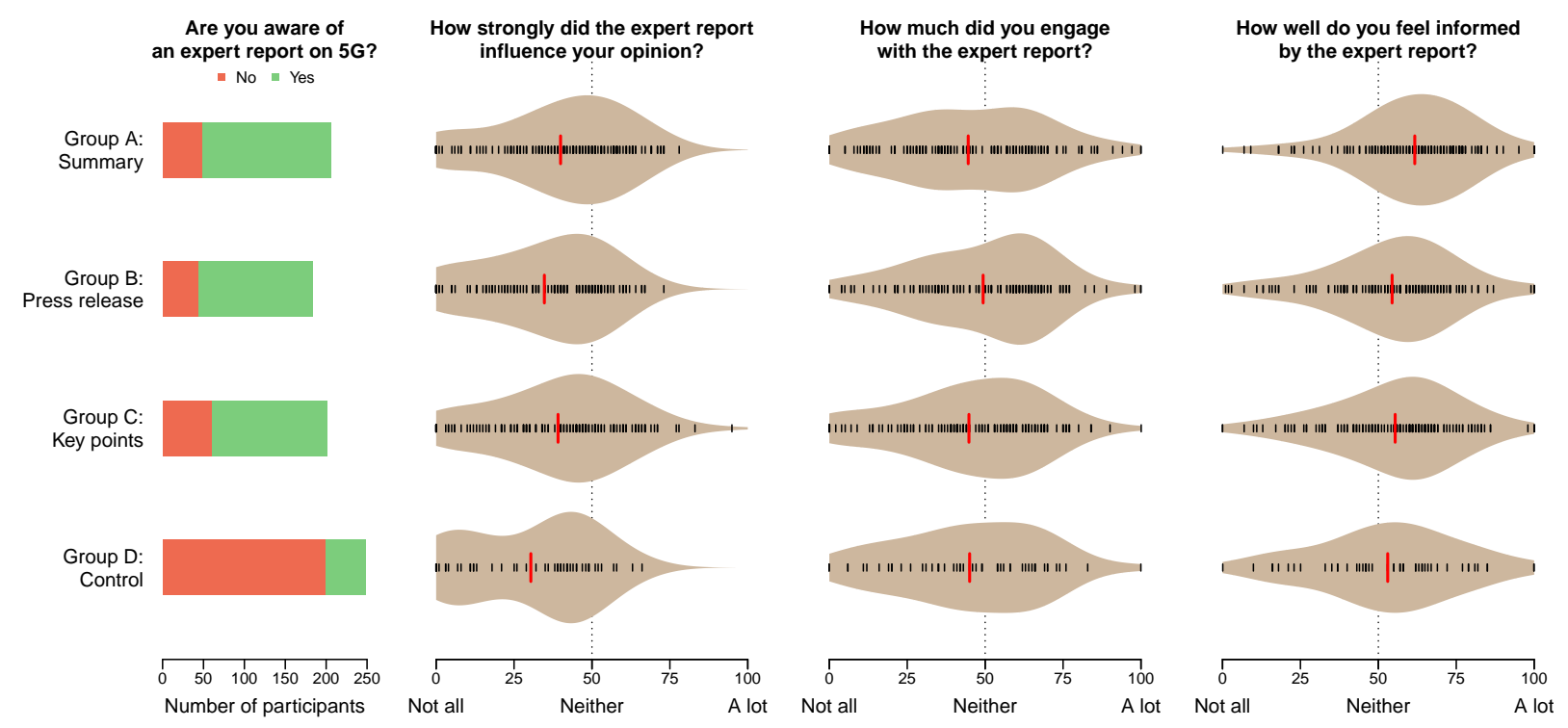

Figure S13: Study 2 (longitudinal sample, $\mathrm{N}=839$ ): Manipulation check of the field experiment and related measures. Only participants who responded with "yes" to the first question were shown the three subsequent questions. 


\section{F Tables}

Table S1: Sociodemographic and related variables

\begin{tabular}{llccc}
\hline \hline & & Study 1 & $\begin{array}{c}\text { Study } 2 \\
\text { cross-sectional }\end{array}$ & $\begin{array}{c}\text { Study } 2 \\
\text { longitudinal }\end{array}$ \\
$\mathrm{N}$ & & & 1013 & \\
Sex & & 2919 & 539 \\
& female & $1432 / 49.1 \%$ & $503 / 49.7 \%$ & $371 / 44.2 \%$ \\
Age & male & $1487 / 50.9 \%$ & $510 / 50.3 \%$ & $468 / 55.8 \%$ \\
Education & & & \\
& & $44.42(\mathrm{SD}=16.14)$ & $44.23(\mathrm{SD}=16.21)$ & $45.28(\mathrm{SD}=16.07)$ \\
& & & & \\
& basic education & $999 / 34.2 \%$ & $329 / 32.5 \%$ & $266 / 31.7 \%$ \\
Occupation & high school & $716 / 24.5 \%$ & $282 / 27.8 \%$ & $206 / 24.6 \%$ \\
& college degree & $1175 / 40.3 \%$ & $393 / 38.8 \%$ & $358 / 42.7 \%$ \\
& none & $29 / 1 \%$ & $9 / 0.9 \%$ & $9 / 1.1 \%$ \\
& in education & $270 / 9.3 \%$ & $102 / 10.1 \%$ & $77 / 9.2 \%$ \\
& employed & $1856 / 63.6 \%$ & $625 / 61.7 \%$ & $529 / 63.1 \%$ \\
& self-employed & $185 / 6.3 \%$ & $75 / 7.4 \%$ & $51 / 6.1 \%$ \\
& house-wm./man & $141 / 4.8 \%$ & $47 / 4.6 \%$ & $43 / 5.1 \%$ \\
& retired & $403 / 13.8 \%$ & $138 / 13.6 \%$ & $124 / 14.8 \%$ \\
& none & $63 / 2.2 \%$ & $26 / 2.6 \%$ & $15 / 1.8 \%$ \\
& private & $1769 / 60.6 \%$ & $612 / 60.4 \%$ & $498 / 59.4 \%$ \\
& professional & $18 / 0.6 \%$ & $10 / 1 \%$ & $10 / 1.2 \%$ \\
& both & $968 / 33.2 \%$ & $340 / 33.6 \%$ & $290 / 34.6 \%$ \\
& no & $164 / 5.6 \%$ & $51 / 5 \%$ & $41 / 4.9 \%$ \\
& computer & $1535 / 52.6 \%$ & $513 / 50.6 \%$ & $465 / 55.4 \%$ \\
& smartphone & $1105 / 37.9 \%$ & $424 / 41.9 \%$ & $299 / 35.6 \%$ \\
& tablet & $278 / 9.5 \%$ & $76 / 7.5 \%$ & $75 / 8.9 \%$ \\
\hline
\end{tabular}




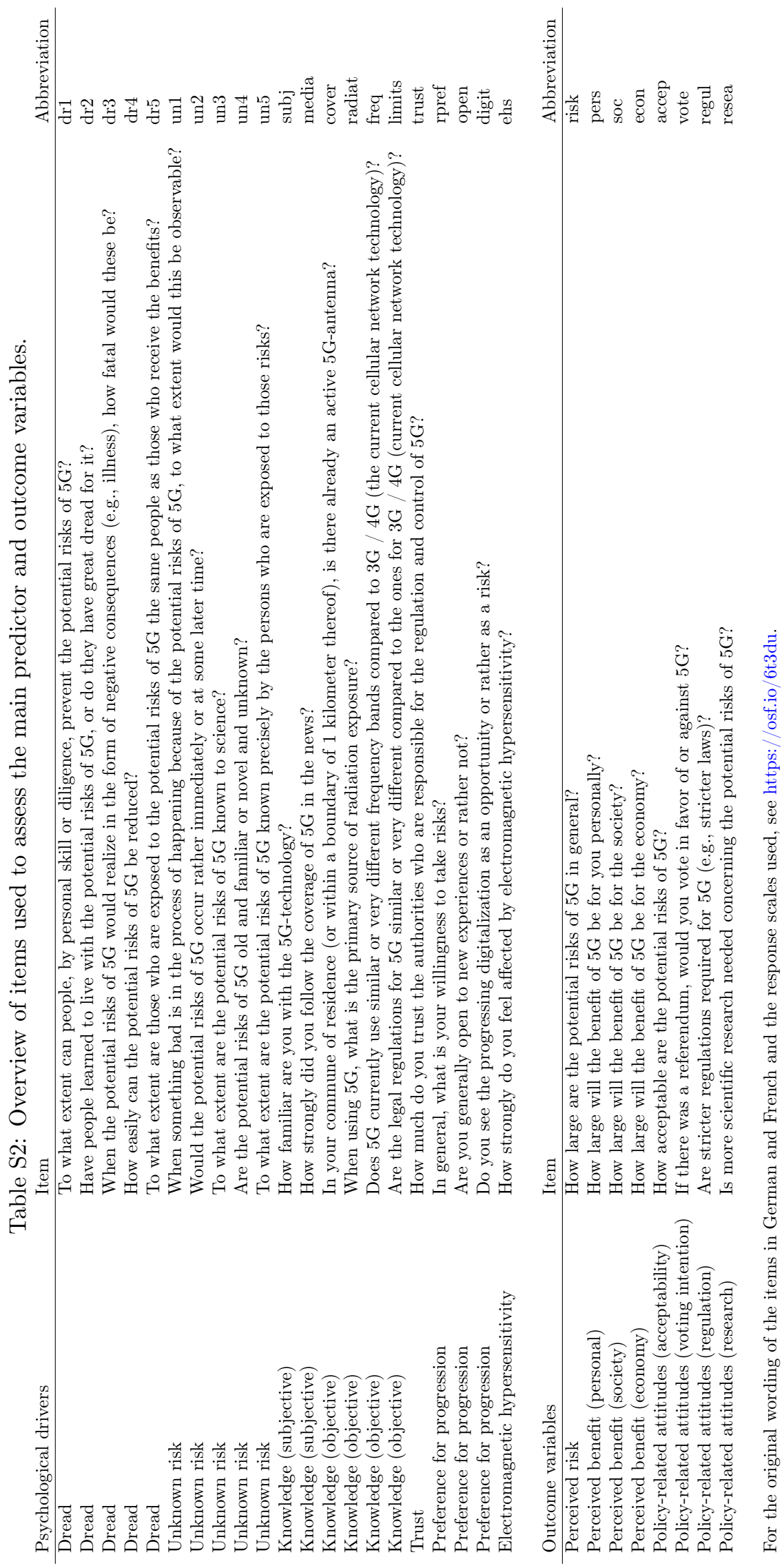




\section{References}

Alhakami, A. S., \& Slovic, P. (1994). A psychological study of the inverse relationship between perceived risk and perceived benefit. Risk Analysis, 14(6), 1085-1096. https://doi.org/10.1111/j.15396924.1994.tb00080.x

Brislin, R. W. (1970). Back-translation for cross-cultural research. Journal of Cross-Cultural Psychology, 1 (3), 185-216. https://doi.org/10.1177/135910457000100301

Clyde, M. A., Ghosh, J., \& Littman, M. L. (2011). Bayesian adaptive sampling for variable selection and model averaging. Journal of Computational and Graphical Statistics, 20(1), 80-101. https://doi.org/ $10.1198 /$ jcgs.2010.09049

Costa, P. T., \& MacCrae, R. R. (1992). Revised NEO personality inventory (NEO PI-R) and NEO five-factor inventory (NEO-FFI): Professional manual. Psychological Assessment Resources, Incorporated.

Epskamp, S., \& Stuber, w. c. f. S. (2017). semPlot: Path diagrams and visual analysis of various SEM packages' output. https://CRAN.R-project.org/package=semPlot

Finucane, M. L., Alhakami, A., Slovic, P., \& Johnson, S. M. (2000). The affect heuristic in judgments of risks and benefits. Journal of Behavioral Decision Making, 13(1), 1-17. https://doi.org/10.1002/(SICI) 1099-0771(200001/03)13:1<1::AID-BDM333>3.0.CO;2-S

Fischhoff, B., Slovic, P., Lichtenstein, S., Read, S., \& Combs, B. (1978). How safe is safe enough? A psychometric study of attitudes towards technological risks and benefits. Policy Sciences, 9(2), 127-152. https://doi.org/10.1007/BF00143739

Frey, R., Pedroni, A., Mata, R., Rieskamp, J., \& Hertwig, R. (2017). Risk preference shares the psychometric structure of major psychological traits. Science Advances, 3, e1701381. https://doi.org/10.1126/ sciadv. 1701381

Hinne, M., Gronau, Q. F., van den Bergh, D., \& Wagenmakers, E.-J. (2019). A conceptual introduction to Bayesian model averaging. PsyArXiv. https://doi.org/10.31234/osf.io/pfynb

$\mathrm{R}$ Core Team. (2019). R: A language and environment for statistical computing. Vienna, Austria. https: //www.R-project.org/

Rosseel, Y. (2012). Lavaan: An R package for structural equation modeling. Journal of Statistical Software, 48(2), 1-36. https://doi.org/10.18637/jss.v048.i02

Siegrist, M. (1999). A causal model explaining the perception and acceptance of gene technology. Journal of Applied Social Psychology, 29(10), 2093-2106. https://doi.org/10.1111/j.1559-1816.1999.tb02297.x

Siegrist, M. (2000). The influence of trust and perceptions of risks and benefits on the acceptance of gene technology. Risk Analysis, 20(2), 195-204. https://doi.org/10.1111/0272-4332.202020

Siegrist, M., Keller, C., Kastenholz, H., Frey, S., \& Wiek, A. (2007). Laypeople's and experts' perception of nanotechnology hazards. Risk Analysis, 27(1), 59-69. https://doi.org/10.1111/j.1539-6924.2006. 00859.x

Slovic, P., Fischhoff, B., \& Lichtenstein, S. (1985). Characterizing perceived risk. In R. W. Kates, C. Hohenemser, \& J. X. Kasperson (Eds.), Perilous Progress: Managing the Hazards of Technology (pp. 91125). Social Science Research Network. Retrieved September 11, 2019, from https://papers.ssrn. $\mathrm{com} /$ abstract $=2185557$

Stan Development Team. (2016). Rstanarm: Bayesian applied regression modeling via Stan. https://mcstan.org

van den Bergh, D., Clyde, M. A., Raj, A., de Jong, T., Gronau, Q. F., Marsman, M., Ly, A., \& Wagenmakers, E.-J. (2020). A tutorial on Bayesian multi-model linear regression with BAS and JASP. PsyArXiv. https://doi.org/10.31234/osf.io/pqju6

Weber, E. U., Blais, A. R., \& Betz, N. E. (2002). A domain-specific risk-attitude scale: Measuring risk perceptions and risk behaviors. Journal of Behavioral Decision Making, 15(4), 263-290. https://doi. $\operatorname{org} / 10.1002 / \mathrm{bdm} .414$ 


\title{
Supplemental Online Materials
}

Psychological drivers of individual differences in risk perception:

A systematic case study focusing on $5 \mathrm{G}$

\author{
Renato Frey \\ University of Basel
}

February 3, 2021 


\section{Contents}

A Detailed methods study $1 \quad 3$

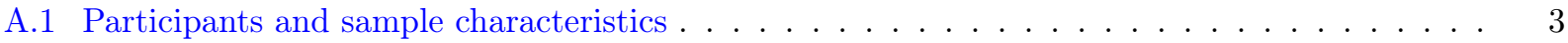

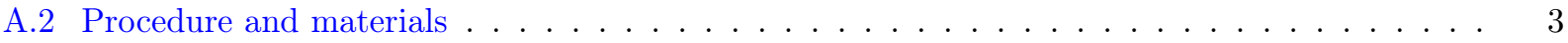

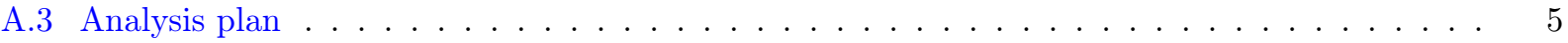

A.4 Deviations from preregistered analysis $\mathrm{plan} \ldots \ldots \ldots \ldots \ldots$

B Extended results study $1 \quad 5$

B.1 Measurement models . . . . . . . . . . . . . . . . . . . . . . . . . 5 5

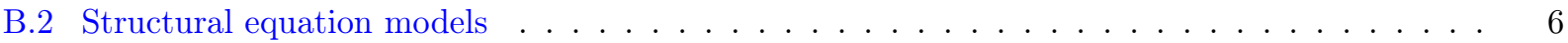

B.3 GIS analysis of network coverage $\ldots \ldots \ldots \ldots \ldots \ldots \ldots \ldots \ldots \ldots$

B.4 Perception of health risks $\ldots \ldots \ldots \ldots \ldots \ldots$

$\begin{array}{ll}\text { C Detailed methods study } 2 & 8\end{array}$

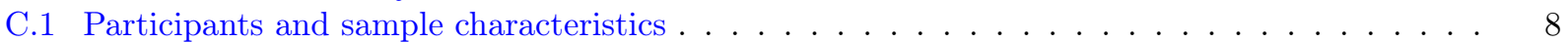

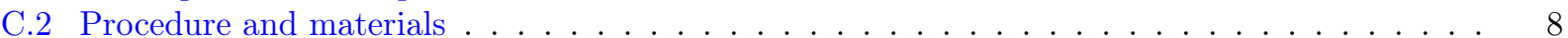

C.3 Expert report on $5 \mathrm{G}$ and experimental conditions in the longitudinal sample $\ldots \ldots \ldots$

C.4 Analysis plan . . . . . . . . . . . . . . . . . . . . . . . 9

$\begin{array}{ll}\text { D Extended results study } 2 & 10\end{array}$

D.1 Cross-validations of study 1 (cross-sectional sample) $\ldots \ldots \ldots \ldots \ldots$

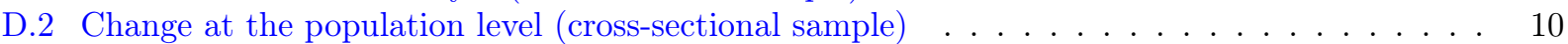

D.3 Intraindividual change in person-specific drivers (longitudinal sample) . . . . . . . . . . . 10

D.4 Associations of intraindividual change in predictor and outcome variables (longitudinal sample) 11

$\begin{array}{llr}\text { E } & \text { Figures } & 12\end{array}$

F Tables 


\section{A Detailed methods study 1}

The institutional review board of the Department of Psychology, University of Basel provided ethical approval (\#021-19-2). The general project rationale and theoretical motivation, the models to be implemented including predicted patterns of associations, and the hypotheses concerning the field experiment (see study 2 ) were preregistered. The preregistrations, dataset, analysis code, and additional materials can be retrieved from https://osf.io/6t3du.

\section{A.1 Participants and sample characteristics}

Study 1 was conducted in November 2019 and involved a large sample of the Swiss general public, representative for gender, four age groups (15-29 years; 30-44 years; 45-59 years; 60-79 years), and two regions (German- and French-speaking parts of Switzerland). Participant recruitment was performed by an external survey company using an existing panel, which exclusively comprised participants who were actively recruited by telephone (i.e., no self-registrations possible). Sampling was conducted using interlocked quotas and slightly exceeded the aspired sample size of 3,000 participants, resulting in $N_{S 1}=3,096$ participants who completed the study. Participants who failed an attention check question (see A.2) were excluded from the dataset, resulting in a final sample of 2,919 participants. Table S1 reports sociodemographic information. Data collection was completed on November 26, 2019; that is, before release of the expert report on 5G by the Swiss Federal Office for the Environment (see C.3). On average it took participants 14 minutes to complete the study; as a compensation, participants earned 100 points (i.e., equivalent to $1 \mathrm{CHF}$ ) in the survey company's incentive program.

\section{A.2 Procedure and materials}

The study was conducted online using a responsive mobile first design (i.e., the study could be completed on all types of devices). All study materials were translated from German to French by the survey company, and participants were free to choose their preferred language at the onset of the study. An independent back-translation (Brislin, 1970) was performed to identify potential changes in meaning, but only minor adjustments needed to be made. Table S2 provides an overview of the items used for the main predictor and outcome variables (translated into English), and the full study materials (i.e., including original items and back translations) are available from https://osf.io/6t3du.

\section{Informed consent and sociodemographic information}

Participants were first informed about the background and purpose of the study and then provided informed consent. Next, they reported sociodemographic information (i.e., gender, age, canton of residence, zip code, level of education, employment status) and indicated whether they owned a smartphone, as well as the type of device they were using to complete the survey.

\section{General risk perception of $5 \mathrm{G}$}

Next, participants either first rated their general risk perception of $5 \mathrm{G}$ and then proceeded to the next block (see "psychometric paradigm" below), or were assigned to the opposite order (i.e., block-randomized design). Building on Fischhoff and colleagues (Fischhoff et al., 1978), general risk perception was assessed with the question: "In your personal view: How large are the potential risks of 5G in general?" Participants responded to this item (as well as all subsequent items) using a continuous slider that yielded values from 0 to 100 . The endpoints and the midpoint of this slider were labeled "very low", "very high", and "medium".

\section{Psychometric paradigm}

The block implementing the psychometric paradigm comprised 10 items (presented in a randomized order), which were primarily selected based on Slovic and colleagues (table 8 of study 3; Slovic et al., 1985), and 
reflected the 5 items loading most strongly on "factor 1" (dread) and the 5 items loading most strongly on "factor 2" (unknown risk). Most of these items were also part of the 9 dimensions originally proposed and implemented by Fischhoff and colleagues (Fischhoff et al., 1978). To illustrate, an item representing factor 1 (dread) was "How easily can the potential risks of $5 \mathrm{G}$ be reduced?", and an item representing factor 2 (unknown risk) was "To what extent are the potential risks of 5G known to science?" See table S2 for the wording of all items.

\section{Attention check}

The previous block also included an attention-check question, prompting participants to move the slider entirely to the left. As specified in the preregistration, the data of participants who failed to do so (i.e., a value higher than 10, thus permitting a small margin of error) were excluded from the analysis.

\section{Perception of health risks}

The next block tapped participants' perceptions of health risks (i.e., arguably the strongest concern related to $5 \mathrm{G}$ in the general public). To be able to compare the degree of $5 \mathrm{G}$ 's perceived health risks with that of other potential health hazards, participants rated their perception of health risks concerning 8 additional technologies and activities (e.g., $3 \mathrm{G} / 4 \mathrm{G}$, the current cellular network technology; smoking; vaccination). To render comparative judgments possible, the 9 items were presented simultaneously and in a randomized order. Furthermore, at the end of this block participants had the opportunity to provide any other risks related to 5G (i.e., beyond health risks) that they were potentially concerned about (i.e., free text input). See B.4 for the results of the analysis on perceived health risks.

\section{Perceived benefits, trust, knowledge, policy-related attitudes}

The next block consisted of 14 items (presented in a randomized order) tapping perceived benefits (separately for personal, social and economic benefits; Fischhoff et al., 1978), trust (single item; cf. Siegrist, 1999, 2000), knowledge (two subjective and four objective indicators), and policy-related attitudes (acceptability of potential risks, voting intention, need for more regulation, need for more scientific research). Subjective knowledge was measured with a subjective rating of one's $5 \mathrm{G}$ knowledge and an item capturing the degree of 5 G-related media consumption. Objective knowledge was measured with questions concerning whether the legal regulations are the same or different as compared to $3 \mathrm{G} / 4 \mathrm{G}$ (i.e., current technology), whether radiation limits are the same or different as compared to $3 \mathrm{G} / 4 \mathrm{G}$, and whether the degree of radiation primarily results from the 5G-antennas or the users' devices. Moreover participants rated their confidence that there exists (vs. that there does not yet exist) an active 5G-antenna in their residential municipality or within a boundary of one kilometer (which was verified using a geographic information system analysis; see B.3 for the results of this analysis and participants' knowledge about 5G coverage). See table S2 for the wording of all items.

\section{Person-related characteristics}

A final block tapped participants' general risk preferences (Frey et al., 2017), their openness to new experiences (Costa \& MacCrae, 1992), whether they predominantly see digitalization as an opportunity or as a potential risk, whether they feel to be affected by electromagnetic hypersensitivity, and their political attitude. The latter was optional to report. See table S2 for the wording of all items.

\section{Additional questions and screening for follow-up}

At the end of study 1, participants were prompted to type in a real-life risky situation (unrelated to the research questions of this article and thus not further reported here) and were asked about their willingness to share their address to receive a brief brochure with information concerning $5 \mathrm{G}$ by mail (see experimental conditions implemented in the longitudinal sample of study 2; C.3). 


\section{A.3 Analysis plan}

All analyses were conducted in $R$ version 3.5.3 (R Core Team, 2019), using the packages lavaan (Rosseel, 2012), semPlot (Epskamp \& Stuber, 2017), and BAS (Clyde et al., 2011) for the main analyses. See https://osf.io/6t3du for the full analysis code. The analysis plan of study 1 involved three main steps.

First, psychometric modeling was conducted to implement measurement models for several of the predictor (i.e., potential drivers of risk perception) and outcome variables (i.e,. perceived risk and benefit, policy-related attitudes), in order to obtain (as reliable as possible) latent variables of the constructs to be measured (see B.1).

Second, for validation reasons the independent measurement models were linked by means of structural equation modeling, to thus examine the associations between particular drivers and outcome variables as to be expected based on previous theory (see B.2).

Third and finally, the main analysis of study 1 was conducted, namely, a Bayesian model comparison testing the predictive power of the various drivers for perceived risk, perceived benefit, and policy-related attitudes. The latent variables extracted from the various measurement models were used as input to this analysis. Multi-model inference analyses were conducted for two reasons: On the one hand, traditional regression analyses typically involve a two-step approach, aimed at first identifying a single-best model, to then interpret this model's parameters for inference. Yet, the uncertainty from the first step (i.e., identification of best model) is ignored in the second step, resulting in overconfident parameter estimates and poor generalizability (van den Bergh et al., 2020). By contrast, model-averaged coefficients (i.e., parameter estimates that are averaged across the entire model space, weighted by the models' posterior probabilities) avoid this problem and provide more reliable estimates (Hinne et al., 2019; van den Bergh et al., 2020). On the other hand, multi-model inference permits quantifying the contributions of each predictor variable to predicting the outcome variables across the entire model space (e.g., posterior inclusion probabilities), as well as to conduct specific comparisons (e.g., relative evidence of the best model over the model that in- / excluded one of the predictors; this is the analysis reported in Fig. 2 of the main text and in figs. S10 and S12). Multi-model inference is thus a particularly promising approach in scenarios such as the current one, where various predictors (i.e., psychological drivers of risk perception) may not be entirely independent from each other; specifically, the uncertainty concerning the predictors' contributions to predicting the outcome variables will be reflected in the posterior model probabilities, and will accordingly be taken into account in the model-averaged estimates. The analyses were conducted using the R-package $B A S$ (Clyde et al., 2011), implementing linear regression models with Gaussian distributions and identity link functions.

\section{A.4 Deviations from preregistered analysis plan}

The analyses deviated in three respects from the preregistered analysis plan. First, the sample was not split into separate exploratory and confirmatory subsamples, because the measurement models were implemented as preregistered (except one minor modification; see section B.1), thus only using confirmatory factor analyses. Second, the R-package BAS (Clyde et al., 2011) was used instead of the BayesFactor package, because the former renders possible to adaptively screen a large number of different model specifications, as well as to extract model-averaged coefficients. Third and relatedly, no specification curve analysis (SCA) was conducted because the $B A S$ package directly renders possible a multiverse analysis, thus making an additional SCA somewhat redundant.

\section{B Extended results study 1}

\section{B.1 Measurement models}

The measurement models were preregistered on https://osf.io/6t3du and the respective items are described in detail in section A.2; see Fig. S3 for an overview and Tab. S2 for the wording. Prior to implementing the various measurement models, Pearson correlations were compared with Spearman correlations across all 
indicators; as the mean (maximum) absolute difference between the two types of correlations was only .008 (.062), all subsequent analyses were conducted based on the former. The fit of the measurement models with more than one indicator was evaluated according to the following criteria: comparative fit index (CFI), Tucker-Lewis index (TLI), root mean square error of approximation (RMSEA), proportion of explained variance $\left(R^{2}\right)$.

Psychological drivers. The two dimensions of the psychometric paradigm were implemented with two measurement models, one for dread risk (DREAD; A in Fig. S3) and one for unknown risk (UNKNO; B in Fig. S3). Both measurement models included five indicators (adapted from the original psychometric paradigm) and achieved satisfactory fits (DREAD: CFI $=.98, \mathrm{TLI}=.96, \mathrm{RMSEA}=.04, R^{2}=.26$; UNKNO: CFI $=$ .9 , TLI $=.81$, RMSEA $=.08, R^{2}=.22$ ). Trust (C in Fig. S3) was implemented with a single indicator (in line with previous research; e.g., Siegrist et al., 2007), namely, the degree of trust in the authorities responsible for regulating $5 \mathrm{G}$. Note that the downside of a single-indicator model may consist of a relatively lower measurement reliability (i.e., depending on the degree of measurement error of the indicator variable), thus potentially resulting in attenuated associations with other variables of interest. For knowledge, it turned out that the two items measuring subjective knowledge (i.e., a subjective rating of one's $5 \mathrm{G}$ knowledge; an item capturing the degree of 5G-related media consumption) correlated only weakly with the four items measuring objective knowledge (i.e., knowledge concerning the main sources of radiation, frequencies in use, legal radiation limits, and current network coverage; see section B.3). Accordingly, a measurement model with two separate factors (KNsubj and KNobj; D in Fig. S3) was implemented, and this solution achieved a satisfactory fit $\left(\mathrm{CFI}=.97\right.$, TLI $\left.=.94, \mathrm{RMSEA}=.03, R^{2}=.33\right)$. This implementation was the only deviance from the preregistered models, and thus no separate exploratory and confirmatory subsamples were used in study 1 (as originally specified in the preregistration, in case there would have been a need for substantial exploratory analyses; note that this model was subsequently also cross-validated in the independent crosssectional sample of study 2; see D.1). The measurement model for a person's general preference for progression (PROGR; E in Fig. S3) included three indicators, namely, a person's risk preference, openness to new experiences, and attitude towards digitalization. This model also achieved a satisfactory fit (CFI $=1$, TLI $=1, \operatorname{RMSEA}=0, R^{2}=.32$ ).

Perceived risk and benefit. Perceived risk (PRISK; F in Fig. S3) was composed of a single indicator (in line with previous research; Fischhoff et al., 1978), namely, participants' rating of their general risk perception of 5G. Perceived benefit (PBENE; G in Fig. S3) summarized the indicators for perceived personal benefit, benefit for society, and benefit for the economy; these three variables correlated highly between each other and resulted in a satisfactory fit of the respective measurement model $\left(\mathrm{CFI}=1, \mathrm{TLI}=1, \mathrm{RMSEA}=0, R^{2}\right.$ $=.61)$.

Policy-related attitudes. Finally, policy-related attitudes (POLICY; H in Fig. S3) was implemented with one measurement model and involved the following indicators: acceptability of risk, voting intention, need for more regulation, and need for more research. This measurement model achieved a satisfactory fit $\left(\mathrm{CFI}=.99, \mathrm{TLI}=.98, \mathrm{RMSEA}=.08, R^{2}=.62\right)$.

The factor scores extracted from the various measurement models (see Fig. S4 for their distributions and Fig. S5 for their inter-correlations) were standardized and constituted the input for the subsequent Bayesian model comparison analyses. To be able to depict the associations between the various predictor and outcome variables on the original response scale (i.e., Fig. 2 and 4 of the main text), as well as to be able to compute intraclass-coefficients using the original response scale, approximations for the factor values were computed. Specifically, for each latent variable with multiple indicators, the latter were unit-weighted and averaged. The resulting scores correlated very highly with the original factor scores, namely, $r=.94$ (dread), .96 (unknown risk), .86 (objective knowledge), .85 (subjective knowledge), and .89 (preference for progression).

\section{B.2 Structural equation models}

Prior to comparing the various potential drivers of risk perception directly with each other (i.e., the main analysis of study 1), four separate structural equation models (SEMs; A-D in Fig. S6) were implemented. 
These SEMs linked the various measurement models and thus permitted validating the measurement models and testing a number of associations predicted by previous theory. The four SEMs were preregistered on https://osf.io/6t3du.

First, according to the psychometric paradigm (A in Fig. S6) dread risk and unknown risk drive the perception of risks (positively) and benefits (negatively), and as a consequence the latter two dimensions may be inversely related. These patterns have repeatedly been observed empirically and in diverse contexts (Alhakami \& Slovic, 1994; Finucane et al., 2000; Fischhoff et al., 1978; Weber et al., 2002). The implementation of the psychometric paradigm at the level of individual participants performed very well, with a CFI $=.94$, a $\mathrm{TLI}=.92$, and a RMSEA $=.06$. Moreover, dread risk was, as predicted, strongly and positively associated with perceived risk, as well as a strongly but negatively associated with perceived benefit. However, the predicted associations of unknown risk did not emerge.

Second, according to the trust-knowledge model (B in Fig. S6), trust and knowledge should both be negatively related to perceived risk but positively related to perceived benefit. The implementation of this model, with separate factors for subjective and objective knowledge (KNsubj and KNobj, respectively; see section B.1), also resulted in a satisfactory fit, namely, a CFI $=.97$, a TLI $=.95$, and a RMSEA $=.05$. Moreover, the model confirmed that trust was strongly and negatively associated with perceived risk, as well as strongly but positively associated with perceived benefit - in line with the predictions. Furthermore, objective knowledge (but not subjective knowledge) was negatively associated with perceived risk, and both were weakly and positively associated with perceived benefit.

Third, a person's general preference for progression (C in Fig. S6), that is, a potentially quite stable person-specific driver, was expected to be negatively associated with perceived risk and to be positively associated with perceived benefit. This model also performed very well, with a CFI $=.97$, a TLI $=.95$, and a RMSEA $=.07$. In line with the predictions, a person's general preference for progression was negatively associated with perceived risk, as well as strongly and positively associated with perceived benefit.

Fourth and finally, perceived risk and benefit were tested concerning their role in shaping policy-related attitudes, with an expected negative influence of perceived risk and a positive influence of perceived benefit (D in Fig. S6). The model performed very well, with a CFI $=.99$, a TLI $=.99$, and a RMSEA $=.05$. Moreover, as expected perceived risk was strongly and negatively associated with policy-related attitudes, whereas perceived benefit proved to be strongly and positively associated with policy-related attitudes.

In sum, all four SEMs achieved satisfactory fits, and most of the estimated paths were strongly in line with the associations to be expected based on previous theory. As these models are non-nested, a direct comparison of the psychological drivers' predictive power is not straightforward, which is why the extracted factor scores were used to conduct an extensive Bayesian model comparison (i.e., the main analysis of study $1)$.

\section{B.3 GIS analysis of network coverage}

Based on publicly available and up to date information on 5G-antenna locations in Switzerland (retrieved from https://data.geo.admin.ch/ch.bakom.mobil-antennenstandorte- $5 \mathrm{~g} /$ ), a geographic information system (GIS) analysis was conducted to determine whether an active 5G-antenna existed in each residential municipality (or within a boundary of one kilometer thereof) - rendering possible to evaluate participants' respective ratings objectively using the reported zip codes.

This analysis revealed that overall participants' knowledge concerning 5G coverage was very limited. Specifically, as can be seen in Figure S7 (first panel) many participants were very unsure about whether 5G coverage existed in their municipality at the time of the study (i.e., many ratings around the mid-point of the scale, indicating maximal uncertainty). Moreover, among participants with actual 5G coverage (second panel), about equally many were convinced that coverage existed as were convinced that coverage did not yet exist (ratings at the extremes of the scale). Among participants without 5G coverage, relatively more participants (correctly) believed that that coverage did not yet exist, whereas only a minority (incorrectly) believed that coverage already existed. 


\section{B.4 Perception of health risks}

To specifically gauge participants' perceptions of health risks associated with 5G (particularly compared to $4 \mathrm{G}$, the current mobile network technology in Switzerland), a comparison of nine potential health hazards was conducted (Fig. S8). According to this analysis, participants perceived 5G to constitute a credibly higher health risk $(M=60.3)$ as opposed to $4 \mathrm{G}\left(M=47.9 ; M_{\Delta}=12.4,95 \%\right.$ highest-density interval [HDI] $=11.1-$ 13.7); despite that in Switzerland the same legal radiation limits apply, and virtually the same frequencies are currently used. Furthermore, on average $5 \mathrm{G}$ was perceived to constitute a substantially higher health risk as compared to vaccinations.

\section{Detailed methods study 2}

Study 2 was identical with study 1, except for four additional questions related to the experimental manipulation (in the longitudinal sample) that was administered in between the two studies (see C.2).

\section{C.1 Participants and sample characteristics}

Study 2 was conducted in January 2020 and involved two separate samples: for the cross-sectional sample, it was aspired to newly recruit 1,000 participants, resulting in a final sample size of $N=1,013$ participants after applying the same criteria as described for study 1. For the longitudinal sample, the goal was to collect retest data for 1,000 participants of study 1 (i.e., 250 participants for each of the four experimental conditions, see C.3). Assuming an attrition rate of about 30\%, 1,400 participants were thus sampled from study 1 (i.e., from all participants who indicated willingness to receive a letter with information on $5 \mathrm{G}$ by mail; $69.5 \%$ of participants). After applying the same criteria as described for study 1, and after removing 130 participants from the treatment groups who were not aware that they had received a letter with information about $5 \mathrm{G}$ from the survey company (i.e., the intervention of the field experiment) a final sample size of $N=839$ participants resulted. Table S1 reports sociodemographic information. Participants received the same compensation as in study 1, and participants in the longitudinal sample received a bonus of 100 points as an additional incentive to also complete study 2 .

\section{C.2 Procedure and materials}

The procedures and materials were identical to those of study 1 , with some additional questions at the end of study 2. Specifically, all participants were asked about their awareness of the published expert report, and in case of an affirmative response, they reported (a) how extensively they had studied the full report or a summary thereof, (b) how well they felt informed by the expert report, and (c) to what extent they believed that their attitudes towards $5 \mathrm{G}$ had changed due to the expert report (Fig. S13). Moreover, as a manipulation check participants in the longitudinal sample were explicitly asked whether they had recently received a letter containing information on $5 \mathrm{G}$ from the survey company.

\section{C.3 Expert report on 5G and experimental conditions in the longitudinal sam- ple}

On November 28, 2019, the Swiss Federal Office for the Environment (FOEN) publicly released an expert report on 5G. This report was developed in an interdisciplinary working group on behalf of the Federal Department for the Environment, Transport, Energy and Communication. The group included representatives of federal offices and cantonal authorities, mobile phone providers, doctors, and representatives of cities and municipalities. Its task was to analyze the needs and risks involved in setting up 5G networks and to develop recommendations. It explains technical facts about $5 \mathrm{G}$, deals with the operation of the Swiss 
mobile radio networks and their regulation, estimates the exposure of the population to non-ionizing radiation, and summarizes the scientific findings on possible health consequences. The report is available from https://www.bafu.admin.ch/5g, including a management summary in English that can be retrieved from https://www.newsd.admin.ch/newsd/message/attachments/59387.pdf. Note that the report sketched different scenarios of how the Swiss mobile radio network could be further developed. However, the working group did not make a specific recommendation concerning which option is best. Rather, the report was meant to serve as an evidence base for informing the general public and for policy making in the Swiss government.

\section{Experimental conditions (longitudinal sample)}

Participants of study 1 who were sampled for the longitudinal track were randomly assigned to one of four between-subjects conditions: There were three treatment conditions (groups $\mathrm{A}-\mathrm{C}$ ) and a control condition (group D). In between the two studies and about one week prior to the data collection of study 2, participants in groups $\mathrm{A}-\mathrm{C}$ received a letter with information concerning the expert report on $5 \mathrm{G}$, which was released by the FOEN on November 28, 2019 (i.e., shortly after the data collection of study 1). Specifically:

- Participants of group A received the official management summary of the report (8 pages long; for the English version, see https://www.newsd.admin.ch/newsd/message/attachments/59387.pdf).

- Participants of group B received the official press release for the report (2 pages long; only available in German and French, see https://www.bafu.admin.ch/bafu/de/home/themen/elektrosmog/mitteilungen. msg-id-77294.html).

- Participants of group C received a list of four key points (1 page long) that were extracted from the management summary. Three of these points summarized the legal situation concerning radiation limits in Switzerland, such as concerning general immission limits (i.e., the same as in the majority of neighboring countries), installation limits, and regulations at places with sensitive use (e.g., schools, hospitals) - and that these regulations thus result in stricter overall limits for non-ionising radiation from mobile communications antennas compared to most European countries. The fourth point summarized technical aspects of $5 \mathrm{G}$, such as that the frequencies currently available for $5 \mathrm{G}$ in Switzerland are comparable with those of $4 \mathrm{G}$ and WLAN. These elements were selected because they represent facts all members of the expert group agreed on, and because they may have an impact on people's trust, experienced dread, or knowledge - which turned out to be important psychological drivers of people's risk perceptions according to the analysis of study 1.

- Participants of group D served as controls and did not receive any information by mail.

The motivation for using these naturalistic information formats were twofold: First, to fully adhere to the publicly available evidence base and to thus test the potential effects of realistic interventions. Second, to maximize the chance of inducing intraindividual change in any of the psychological drivers (e.g., dread, trust, knowledge), as the most concise and accessible format (the 1-page with key points) may boost, for instance, trust and knowledge for some persons, whereas for others a more extensive information format (the detailed 8-page summary) may be required to this end, with the short format possibly even triggering the opposite effect.

\section{C.4 Analysis plan}

In study 2 , the analysis plan involved multiple steps for each of the two independent samples.

\section{Cross-sectional sample: Cross-validations and mean-level change in the population}

Using the new (i.e., cross-sectional) sample of participants in study 2, the implementation of the measurement models and the analyses of the drivers of interindividual differences (as conducted in study 1) were first replicated as a robustness check, in order to cross-validate these results (see D.1). Second, the cross-sectional sample was also used to gauge whether any mean-level changes in perceived risk and benefit, as well as in policy-related attitudes, occurred in the population - that is, during the time period between study 1 and study 2 (e.g., due to the public release of the national expert report and associated media coverage; see D.2). 


\section{Longitudinal sample: Field experiment and intraindividual change}

In the longitudinal sample, the degree of intraindividual change in the various drivers of risk perceptions was first assessed (see the main text for the change in the hazard-related drivers, and D.3 for the change in person-specific drivers). Next, Bayesian regression analyses were conducted to examine whether any of the experimental conditions triggered systematic mean-level differences in i) the potential drivers of risk perception, and ii) the various outcome variables. Finally, a Bayesian model comparison analogous to that of study 1 - yet based on the $\Delta$ 's of the predictor and outcome variables - was conducted to examine whether intraindividual change in any of the psychological drivers systematically related to intraindividual change in the outcome variables (see D.4).

\section{Extended results study 2}

\section{D.1 Cross-validations of study 1 (cross-sectional sample)}

Before turning to the main research question of the second study, the data of the newly recruited crosssectional sample was used for two robustness checks. First, the same measurement models as in study 1 were implemented for the various predictor and outcome variables. As Figure S9 shows, the respective model fits and estimated paths were highly congruent with those observed in study 1 (see Fig. S3) - thus corroborating the robustness of these measurement models.

Second, the same Bayesian model comparison as in the main analysis of study 1 was implemented. The results are depicted in Figure S10 and are closely in line with those of study 1 (Fig. 3 of the main text). The only notable exceptions included that gender differences were less pronounced for perceived risk and policy-related attitudes, and there was no longer an effect of education on perceived benefit. Note that the Bayes Factors generally tended to be lower due to the smaller sample size of the cross-sectional sample (i.e., as compared to the sample of study 1).

\section{D.2 Change at the population level (cross-sectional sample)}

Moreover, the cross-sectional sample of study 2 was used to conduct independent-groups comparisons (i.e., between the participants of study 1 and participants of study 2), using the raw ratings of perceived risk, perceived benefit (three separate ratings), and policy-related attitudes (four separate ratings) as dependent variables. These comparisons were conducted by means of Bayesian regression models using rstanarm (Stan Development Team, 2016) and indicated that - at the aggregate level-perceived risk and benefit as well as policy-related attitudes remained virtually unchanged. Specifically, as can be seen in Figure S11, the blue vertical lines in the background depicting the mean differences were all very close to zero. There was only a credible (but small) mean difference across the two studies for perceived benefit for society (i.e., a mean reduction of -2.5 in study $2 ; 95 \% \mathrm{HDI}=-4.4$ to -.6 ). Thus, overall the publication of the expert report did not trigger any substantial mean-level changes in the population during the period between the two studies.

\section{D.3 Intraindividual change in person-specific drivers (longitudinal sample)}

In contrast to hazard-related drivers, person-specific drivers tend to remain relatively stable over time by definition - thus resulting in only modest (or even no) intraindividual change. To verify, intraclass-coefficients (ICCs) were computed for each of the respective continuous variables, and Cohen's kappas were computed for the nominal and ordinal variables. ICCs quantify the degree of intraindividual variance relative to the variance between individuals: An ICC of 1 represents no intraindividual variability, an ICC of 0 represents pure intra- and no interindividual variability, and an ICC of .5 represent the same degree of variability between individuals as within individuals. Conversely, Cohen's kappa reflects a direct measure of accordance. As to be expected, age had an ICC of 1; gender had a kappa of .98 (thus reflecting a very small degree of response error); occupation had a kappa of .87; political attitude had an ICC of .88, education had a kappa of .78, preference for progression had an ICC of .76; and electromagnetic hypersensitivity (EHS) had an ICC 
of .75 (all values were computed for participants of the control group). Thus, by and large the person-specific drivers remained highly stable over time.

\section{D.4 Associations of intraindividual change in predictor and outcome variables (longitudinal sample)}

The results of this analysis are reported in the main text, see Figure S12 for a graphical depiction. As there was hardly any intraindividual change in the person-specific drivers (as expected; see D.3), these did not account for any intraindividual changes in perceived risk and benefit, nor in policy-related attitudes (Fig. S12). 


\section{E Figures}
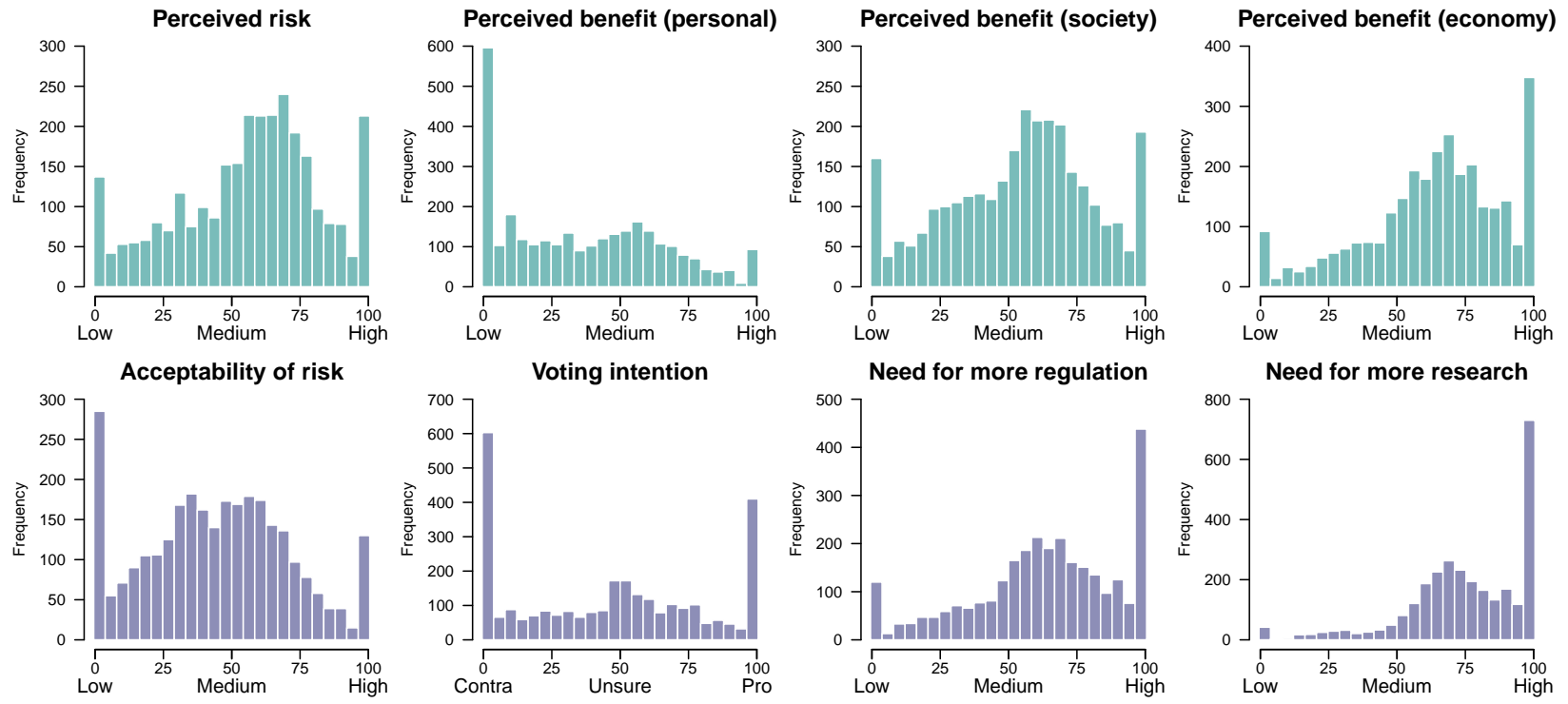

Figure S1: Study $1(\mathrm{~N}=2,919)$ : Distributions of perceived risk and benefit (upper panel) and policy-related attitudes (lower panel). See table S2 for the wording of the items. 


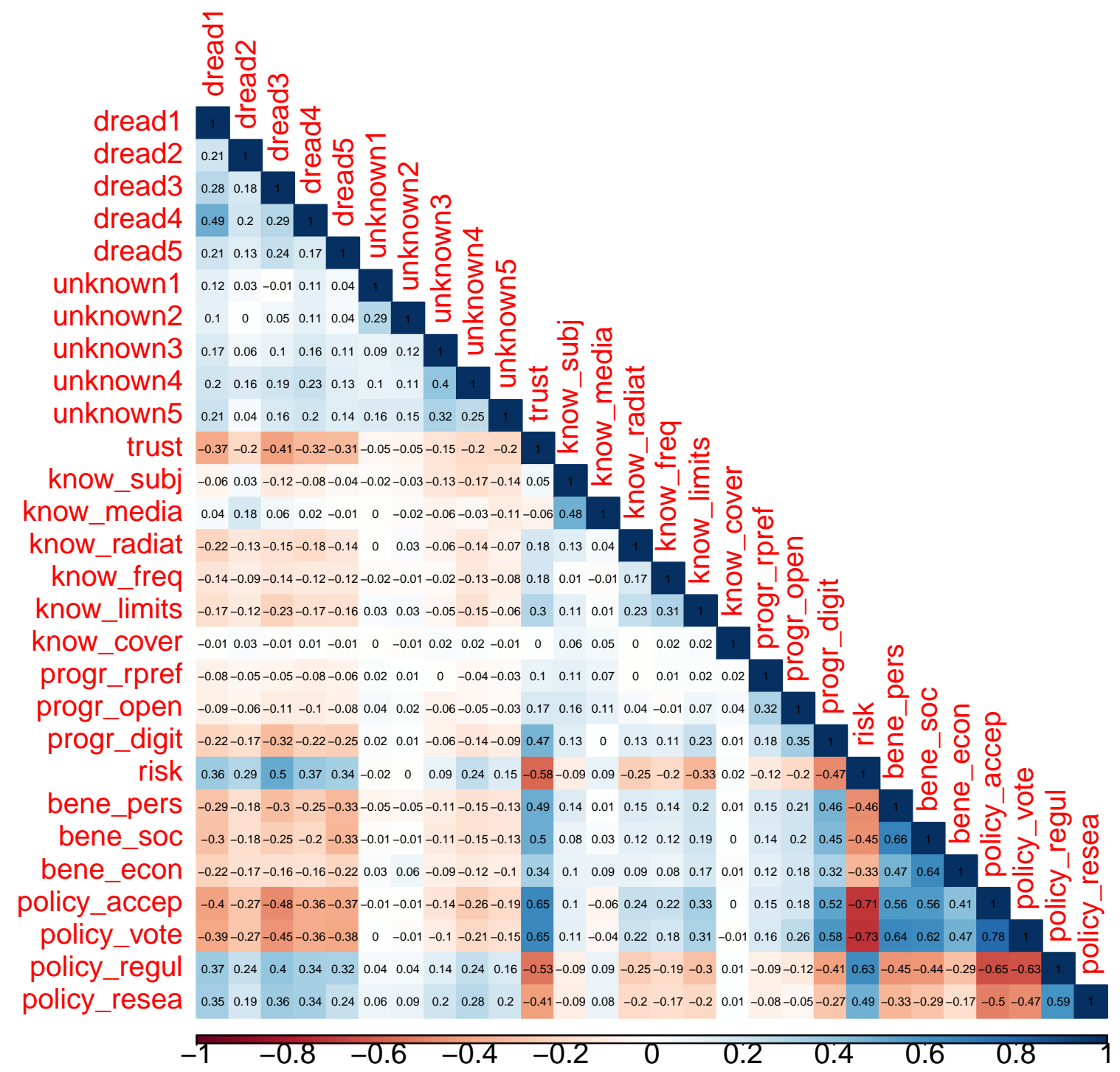

Figure S2: Study $1(\mathrm{~N}=2,919)$ : Correlations between the main variables that were part of the various measurement models (see Fig. S3). See table S2 for the abbreviations and wording of the items. 


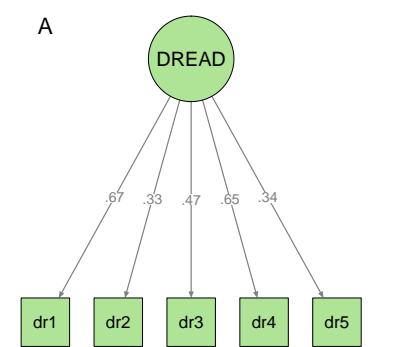

$\mathrm{CFI}=0.98|\mathrm{TLI}=0.96| \mathrm{RMSEA}=0.04 \mid \mathrm{R} 2=0.26$

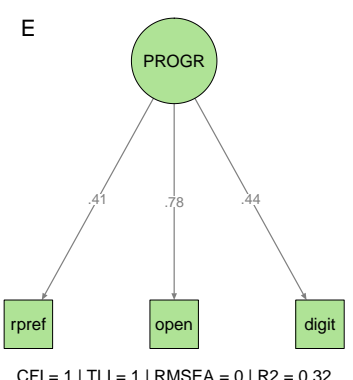

B

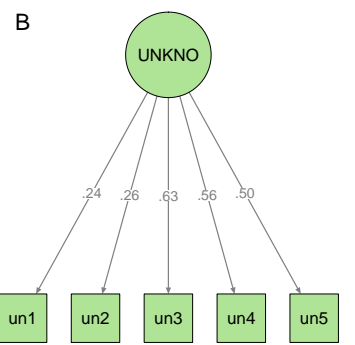

$\mathrm{CFI}=0.9|\mathrm{TLI}=0.81| \mathrm{RMSEA}=0.08 \mid \mathrm{R} 2=0.22$

$\mathrm{F}$

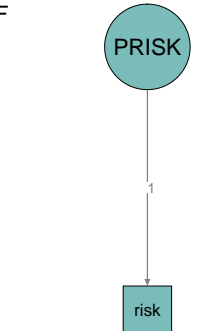

$\mathrm{CFI}=1|\mathrm{TL}|=1|\mathrm{RMSEA}=0| \mathrm{R} 2=1$
C

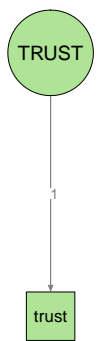

$\mathrm{CFI}=1|\mathrm{TLI}=1| \mathrm{RMSEA}=0 \mid \mathrm{R} 2=1$

$$
\text { G }
$$

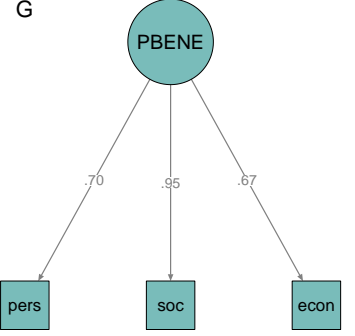

$\mathrm{CFI}=1|\mathrm{TLI}=1| \mathrm{RMSEA}=0 \mid \mathrm{R} 2=0.61$

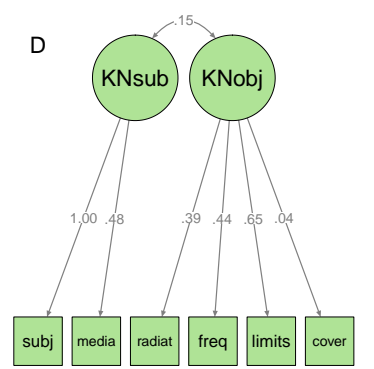

$\mathrm{CFI}=0.97|\mathrm{TLI}=0.94| \mathrm{RMSEA}=0.03 \mid \mathrm{R} 2=0.33$

$\mathrm{H}$

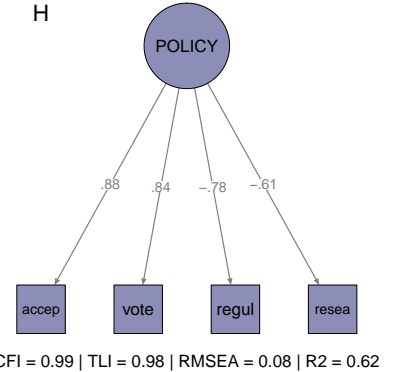

Figure S3: Study $1(\mathrm{~N}=2,919)$ : Measurement models that were implemented using confirmatory factor analyses. The models for the various psychological drivers are depicted in green, and the models for the outcome variables in blue (perceived risk and benefit) and purple (policy-related attitudes). Panel A: DREAD $=$ dread risk. Panel B: UNKNO $=$ unknown risk. Panel C: TRUST $=$ trust. Panel D: KNsubj $=$ knowledge (subjective); KNobj $=$ knowledge (objective). Panel $\mathrm{E}:$ PROGR $=$ preference for progression. Panel $\mathrm{F}$ : PRISK $=$ perceived risk. Panel G: PBENE $=$ perceived benefit. Panel H: POLICY $=$ policy-related attitudes. See section A.2 for a detailed description and table S2 for the abbreviations and wording of the items. 


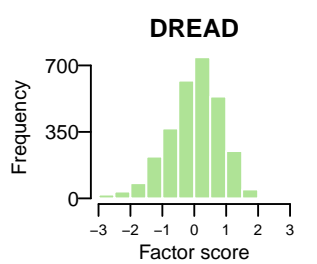

PROGR
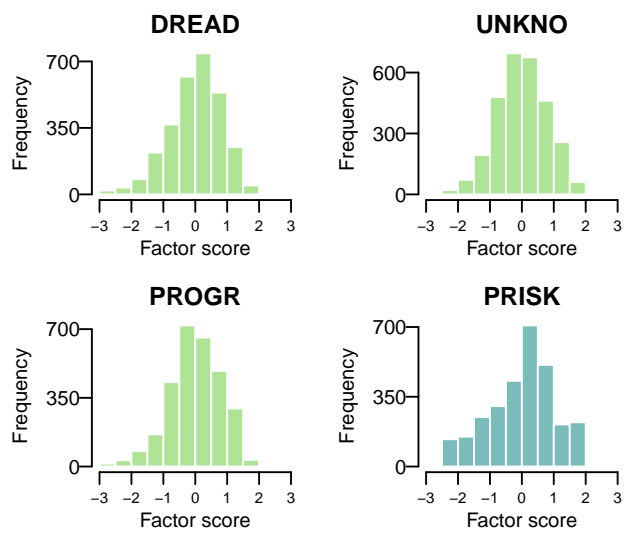

PRISK
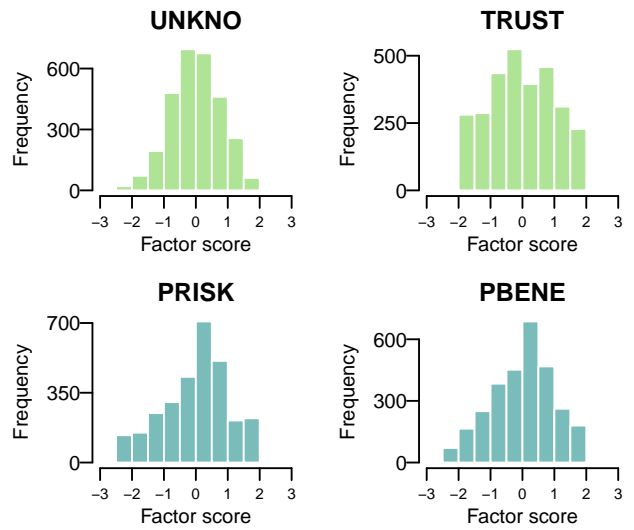

PBENE
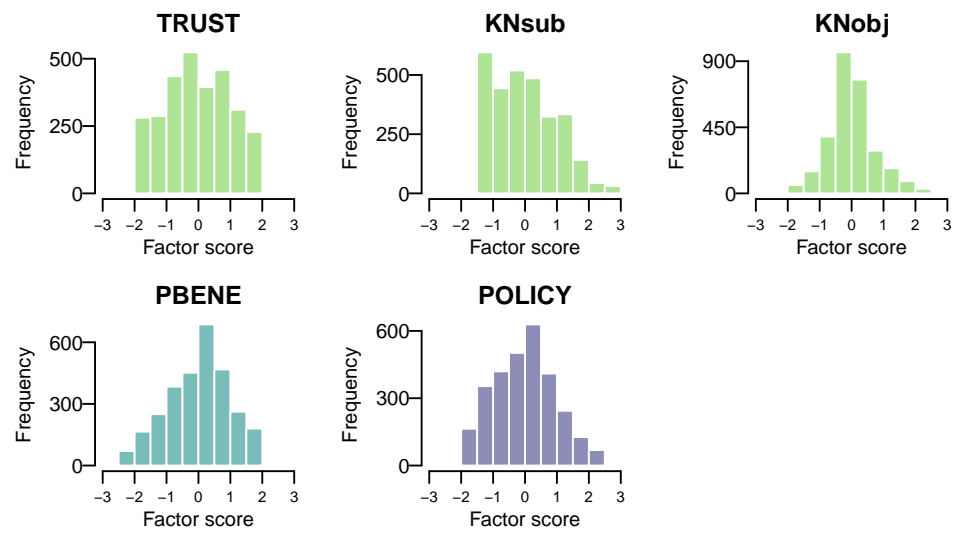

POLICY

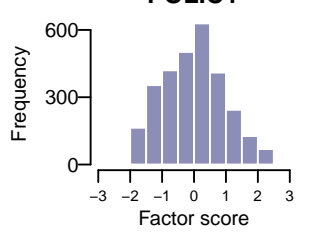

Figure S4: Study $1(\mathrm{~N}=2,919)$ : Distributions of factor scores extracted from the various measurement models (see Fig. S3 for the measurement models and respective abbreviations of the latent variables). 


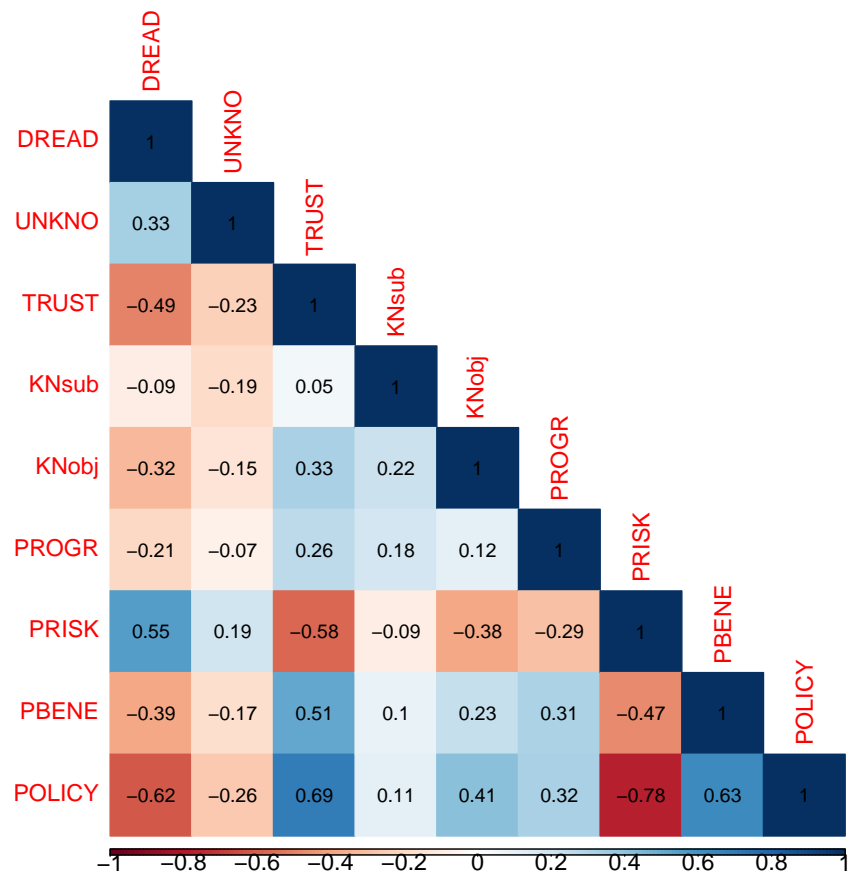

Figure S5: Study $1(\mathrm{~N}=2,919)$ : Correlations between factor scores that were extracted from the various measurement models (see Fig. S3 for the measurement models and respective abbreviations of the latent variables). 
A

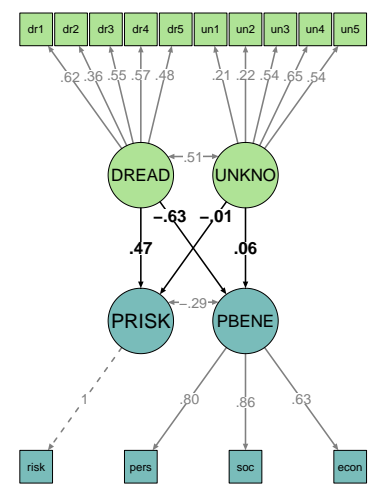

$\mathrm{CFI}=0.94|\mathrm{TLI}=0.92| \mathrm{RMSEA}=0.06$
$\mathrm{B}$

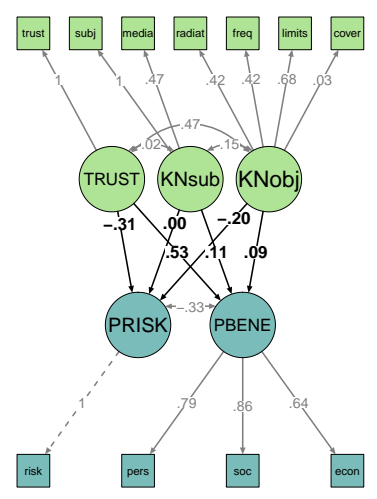

$\mathrm{CFI}=0.97|\mathrm{TLI}=0.95| \mathrm{RMSEA}=0.05$
C

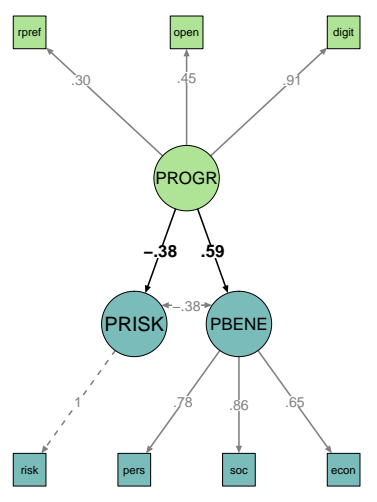

$\mathrm{CFI}=0.97|\mathrm{TLI}=0.95| \mathrm{RMSEA}=0.07$
$\mathrm{D}$

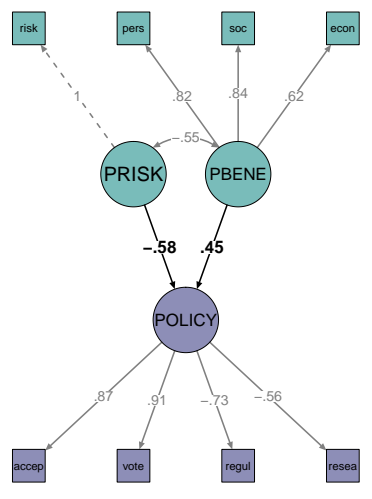

$\mathrm{CFI}=0.99|\mathrm{TLI}=0.99| \mathrm{RMSEA}=0.05$

Figure S6: Study 1 ( $\mathrm{N}=2,919)$ : Four separate structural equation models (SEMs) that linked the various measurement models (see Fig. S3 for the measurement models and table S2 for the abbreviations and wording of the items). These SEMs tested a number of associations to be expected based on previous theory: Panel A depicts the psychometric paradigm involving dread and unknown risk (i.e., hazard-related drivers), panel $\mathrm{B}$ depicts the trust-knowledge model (i.e., hazard-related drivers), panel $\mathrm{C}$ depicts the model testing the role of a person's general preference for progression (i.e., person-specific driver), and panel D depicts the model testing perceived risk and benefit as predictors of policy-related attitudes. 

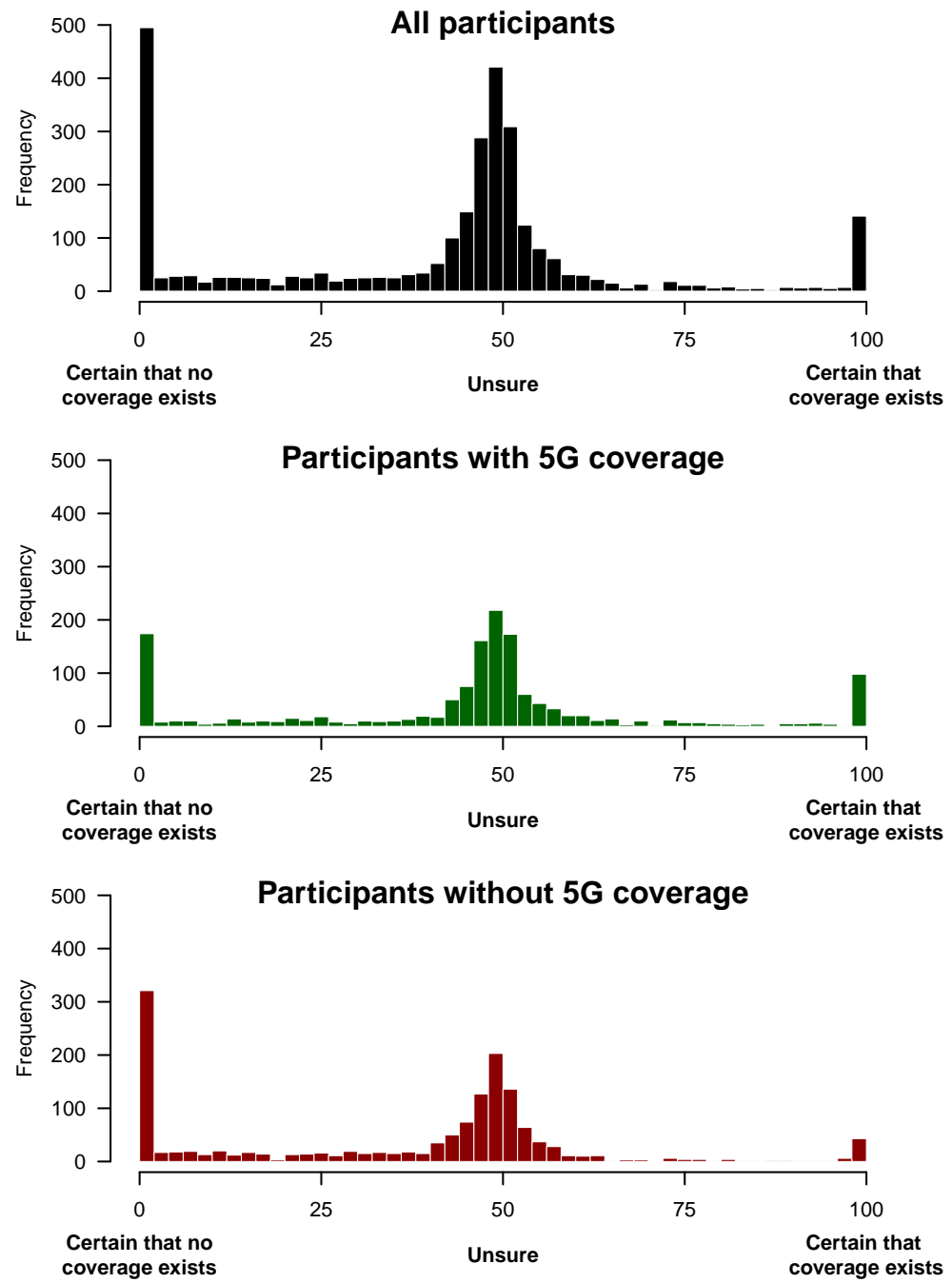

Figure S7: Study $1(\mathrm{~N}=2,919)$ : Participants' objective knowledge about 5G coverage. The ratings express participants' beliefs that $5 \mathrm{G}$ coverage existed at their municipality of residence or within a boundary of 1 kilometer thereof (i.e., at the time of the study). Actual network coverage was determined by means of a GIS-analysis using publicly available data on 5G-antenna locations (see B.3) and participants' zip codes. The panel on the top represents all participants, the panel in the middle represents participants with $5 G$ coverage according to the GIS-analysis, and the bottom panel depicts participants who did not yet have $5 \mathrm{G}$ coverage according to the GIS-analysis. 
Perception of health risks

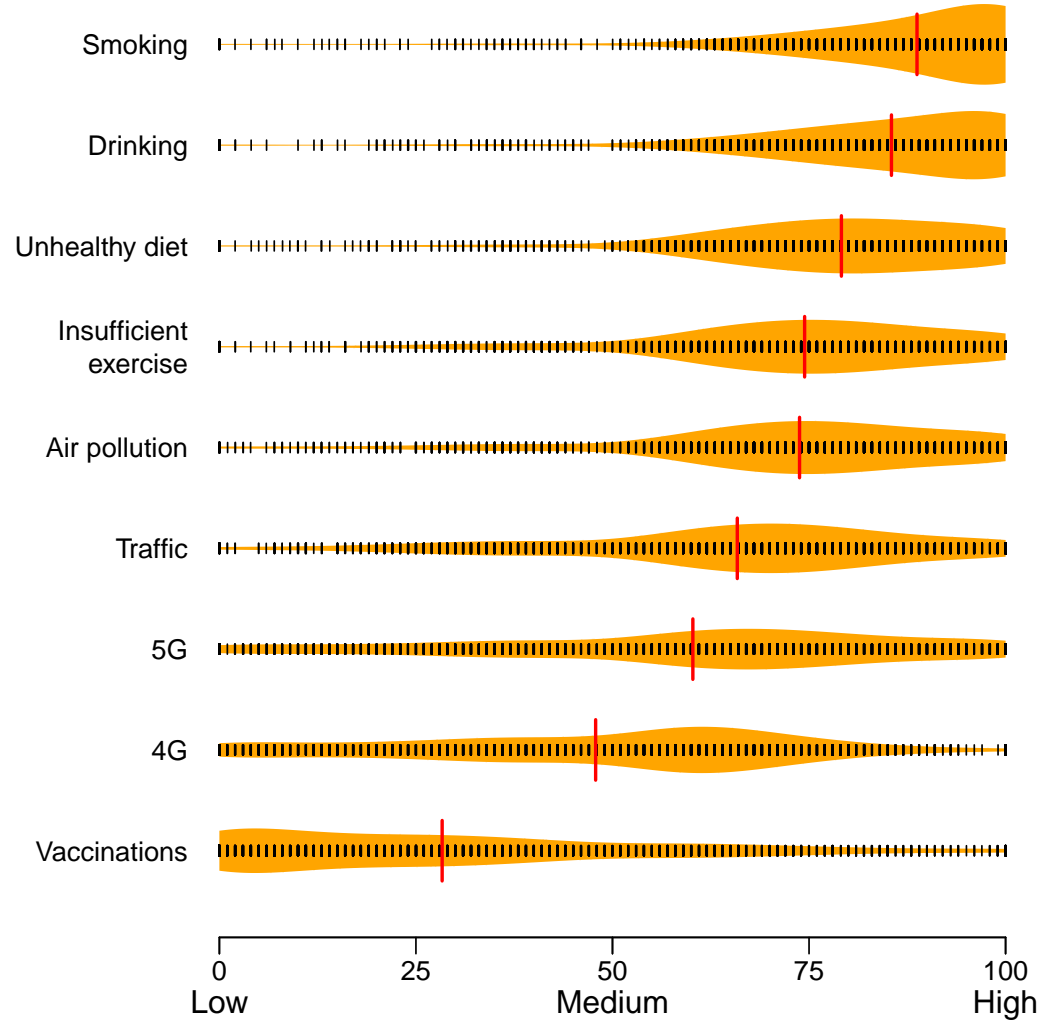

Figure S8: Study $1(\mathrm{~N}=2,919)$ : Comparison of perceived health risks. Each black vertical line represents the rating of one participant (small jitter added), and the red lines depict mean ratings. 


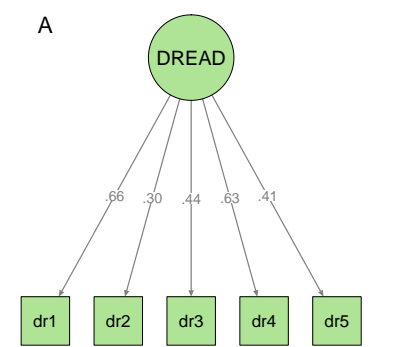

$\mathrm{CFI}=0.98|\mathrm{TL}|=0.97|\mathrm{RMSEA}=0.04| \mathrm{R} 2=0.26$

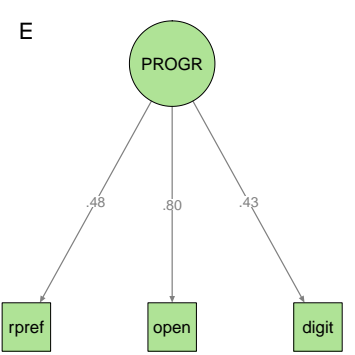

$\mathrm{CFI}=1|\mathrm{TLI}=1| \mathrm{RMSEA}=0 \mid \mathrm{R} 2=0.35$
B

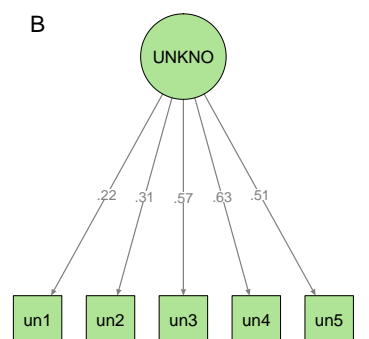

$\mathrm{CFI}=0.87|\mathrm{TLI}=0.74| \mathrm{RMSEA}=0.1 \mid \mathrm{R} 2=0.22$

$\mathrm{F}$

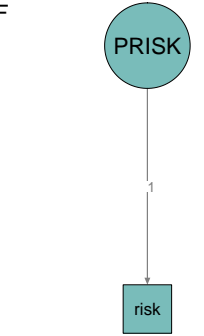

$\mathrm{CFI}=1|\mathrm{TLI}=1| \mathrm{RMSEA}=0 \mid \mathrm{R} 2=1$
C

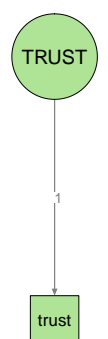

$\mathrm{CFI}=1|\mathrm{TL}|=1|\mathrm{RMSEA}=0| \mathrm{R} 2=1$

$$
\text { G }
$$

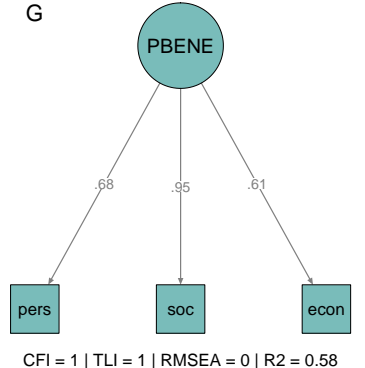

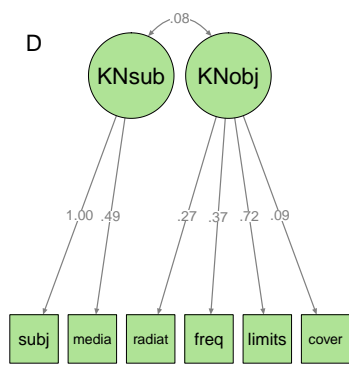

$\mathrm{CFI}=0.86|\mathrm{TLI}=0.77| \mathrm{RMSEA}=0.07 \mid \mathrm{R} 2=0.33$

$\mathrm{H}$

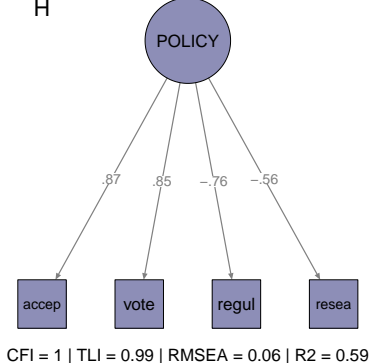

Figure S9: Study 2 (cross-sectional sample, $N=1,013)$ : Measurement models that were implemented as a robustness check of study 1 . Panel A: DREAD = dread risk. Panel B: UNKNO = unknown risk. Panel C: TRUST $=$ trust. Panel D: KNsubj $=$ knowledge (subjective) KNobj $=$ knowledge (objective). Panel $\mathrm{E}: \mathrm{PROGR}=$ preference for progression. Panel F: PRISK $=$ perceived risk. Panel G: PBENE = perceived benefit. Panel H: POLICY = policy-related attitudes. See Figure S3 for the equivalent models originally implemented in study 1, and table S2 for the abbreviations and wording of the items. 


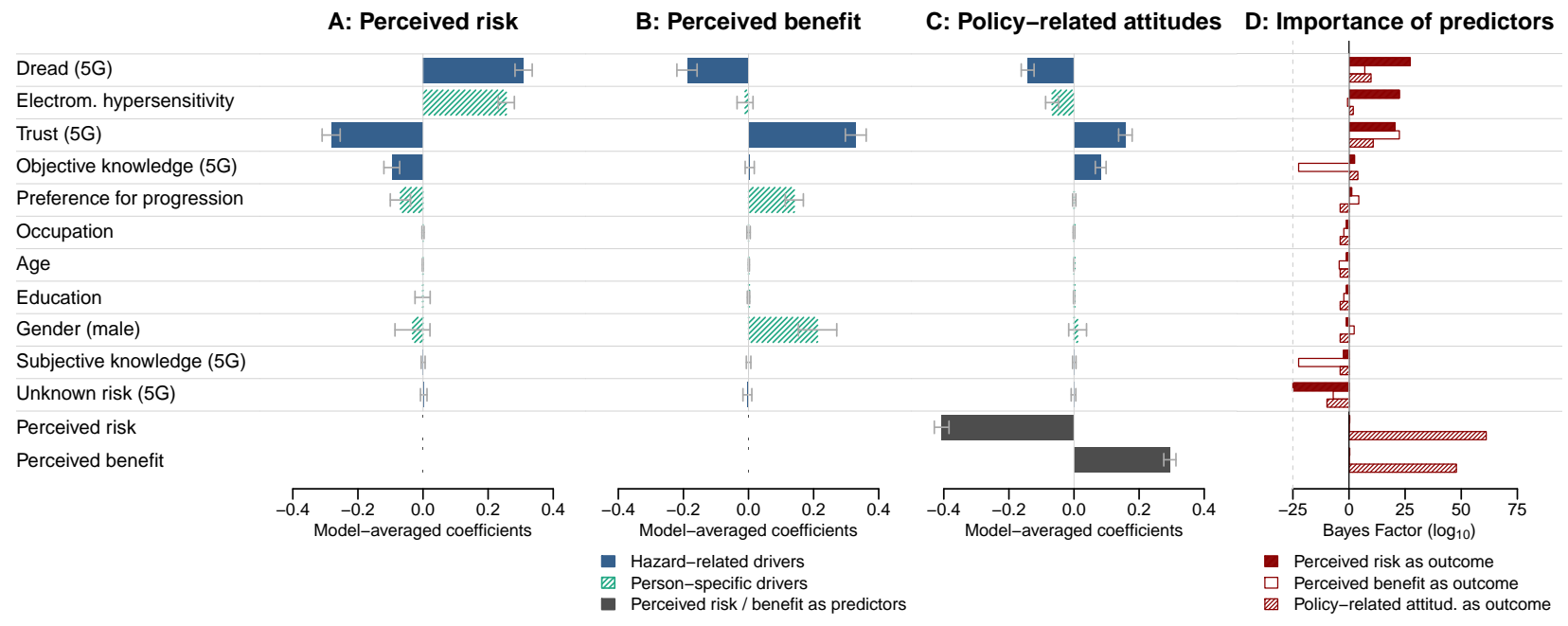

Figure S10: Study 2 (cross-sectional sample, $\mathrm{N}=1,009$ after four list-wise deletions due to missing values): Results of the Bayesian model comparison that was implemented in the independent (i.e., cross-sectional) sample of study 2 as a robustness check of study 1 (see Fig. 3 in the main text). Panel A depicts the coefficients for perceived risk as outcome variable, panel B depicts the coefficients for perceived benefit as outcome variable, and panel $\mathrm{C}$ depicts the coefficients for policy-related attitudes as outcome variable. Panel D depicts the Bayes Factors of all predictor variables, separately for the three outcome variables. 

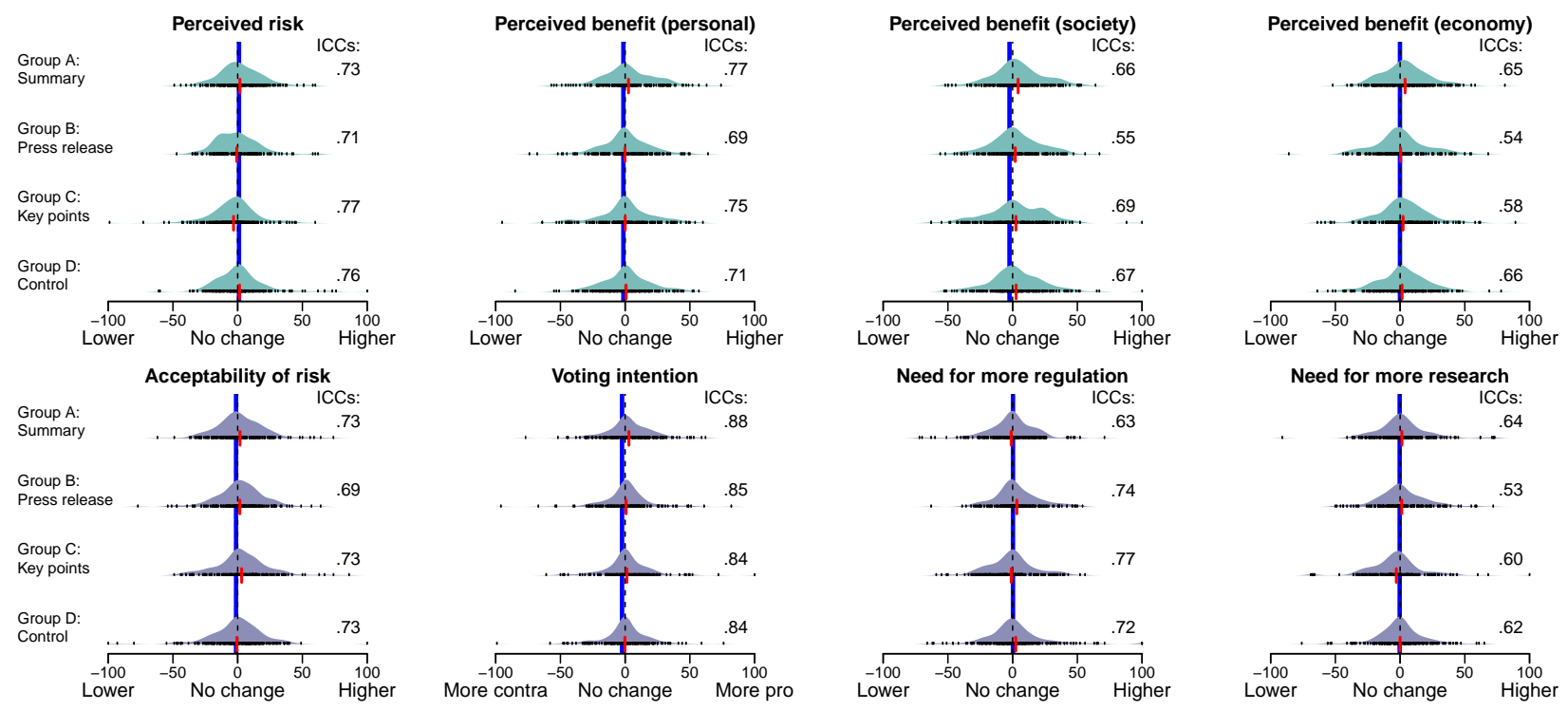

Figure S11: Distributions of intraindiviudal change (across the two studies) in the main outcome variables, separately for the four experimental conditions. The red vertical lines depict average changes per variable and experimental condition (longitudinal sample, $\mathrm{N}=839$ ). The blue vertical lines in the background depict the mean-level change from study 1 to study 2 in the population (i.e., cross-sectional sample, $\mathrm{N}=1,013$ ). 


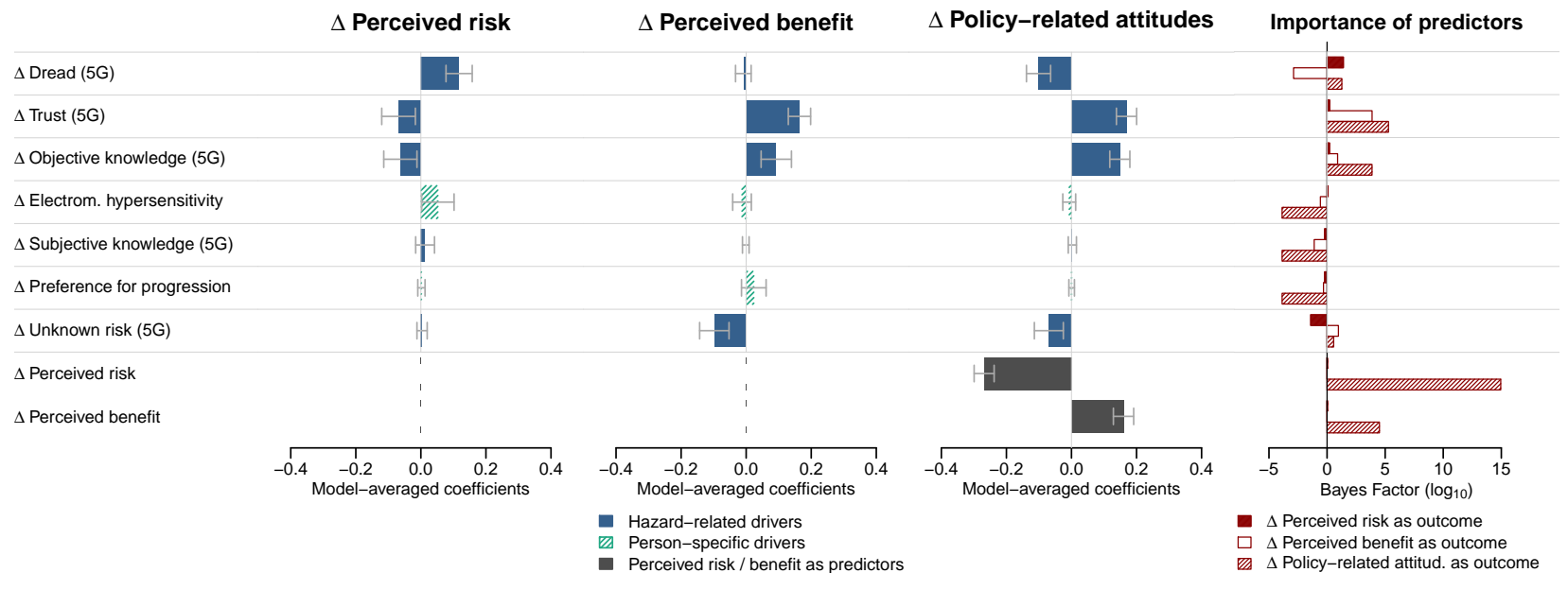

Figure S12: Study 2 (longitudinal sample, $\mathrm{N}=839$ ): Results of the Bayesian model comparison based on intraindividual changes (i.e., $\Delta$ ) in the predictor and outcome variables. 

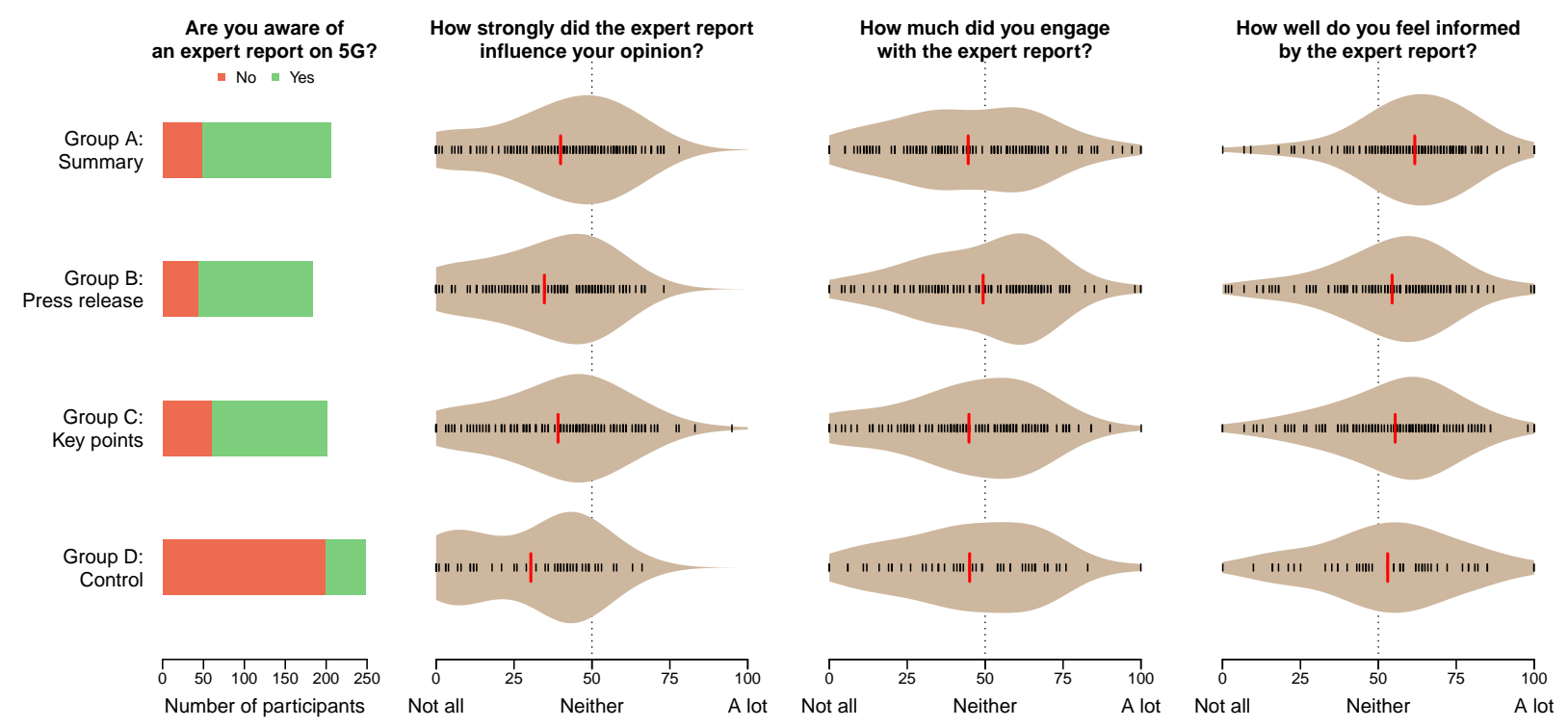

Figure S13: Study 2 (longitudinal sample, $\mathrm{N}=839$ ): Manipulation check of the field experiment and related measures. Only participants who responded with "yes" to the first question were shown the three subsequent questions. 


\section{F Tables}

Table S1: Sociodemographic and related variables

\begin{tabular}{llccc}
\hline \hline & & Study 1 & $\begin{array}{c}\text { Study } 2 \\
\text { cross-sectional }\end{array}$ & $\begin{array}{c}\text { Study } 2 \\
\text { longitudinal }\end{array}$ \\
$\mathrm{N}$ & & & 1013 & \\
Sex & & 2919 & 539 \\
& female & $1432 / 49.1 \%$ & $503 / 49.7 \%$ & $371 / 44.2 \%$ \\
Age & male & $1487 / 50.9 \%$ & $510 / 50.3 \%$ & $468 / 55.8 \%$ \\
Education & & & \\
& & $44.42(\mathrm{SD}=16.14)$ & $44.23(\mathrm{SD}=16.21)$ & $45.28(\mathrm{SD}=16.07)$ \\
& & & & \\
& basic education & $999 / 34.2 \%$ & $329 / 32.5 \%$ & $266 / 31.7 \%$ \\
Occupation & high school & $716 / 24.5 \%$ & $282 / 27.8 \%$ & $206 / 24.6 \%$ \\
& college degree & $1175 / 40.3 \%$ & $393 / 38.8 \%$ & $358 / 42.7 \%$ \\
& none & $29 / 1 \%$ & $9 / 0.9 \%$ & $9 / 1.1 \%$ \\
& in education & $270 / 9.3 \%$ & $102 / 10.1 \%$ & $77 / 9.2 \%$ \\
& employed & $1856 / 63.6 \%$ & $625 / 61.7 \%$ & $529 / 63.1 \%$ \\
& self-employed & $185 / 6.3 \%$ & $75 / 7.4 \%$ & $51 / 6.1 \%$ \\
& house-wm./man & $141 / 4.8 \%$ & $47 / 4.6 \%$ & $43 / 5.1 \%$ \\
& retired & $403 / 13.8 \%$ & $138 / 13.6 \%$ & $124 / 14.8 \%$ \\
& none & $63 / 2.2 \%$ & $26 / 2.6 \%$ & $15 / 1.8 \%$ \\
& private & $1769 / 60.6 \%$ & $612 / 60.4 \%$ & $498 / 59.4 \%$ \\
& professional & $18 / 0.6 \%$ & $10 / 1 \%$ & $10 / 1.2 \%$ \\
& both & $968 / 33.2 \%$ & $340 / 33.6 \%$ & $290 / 34.6 \%$ \\
& no & $164 / 5.6 \%$ & $51 / 5 \%$ & $41 / 4.9 \%$ \\
& computer & $1535 / 52.6 \%$ & $513 / 50.6 \%$ & $465 / 55.4 \%$ \\
& smartphone & $1105 / 37.9 \%$ & $424 / 41.9 \%$ & $299 / 35.6 \%$ \\
& tablet & $278 / 9.5 \%$ & $76 / 7.5 \%$ & $75 / 8.9 \%$ \\
\hline
\end{tabular}




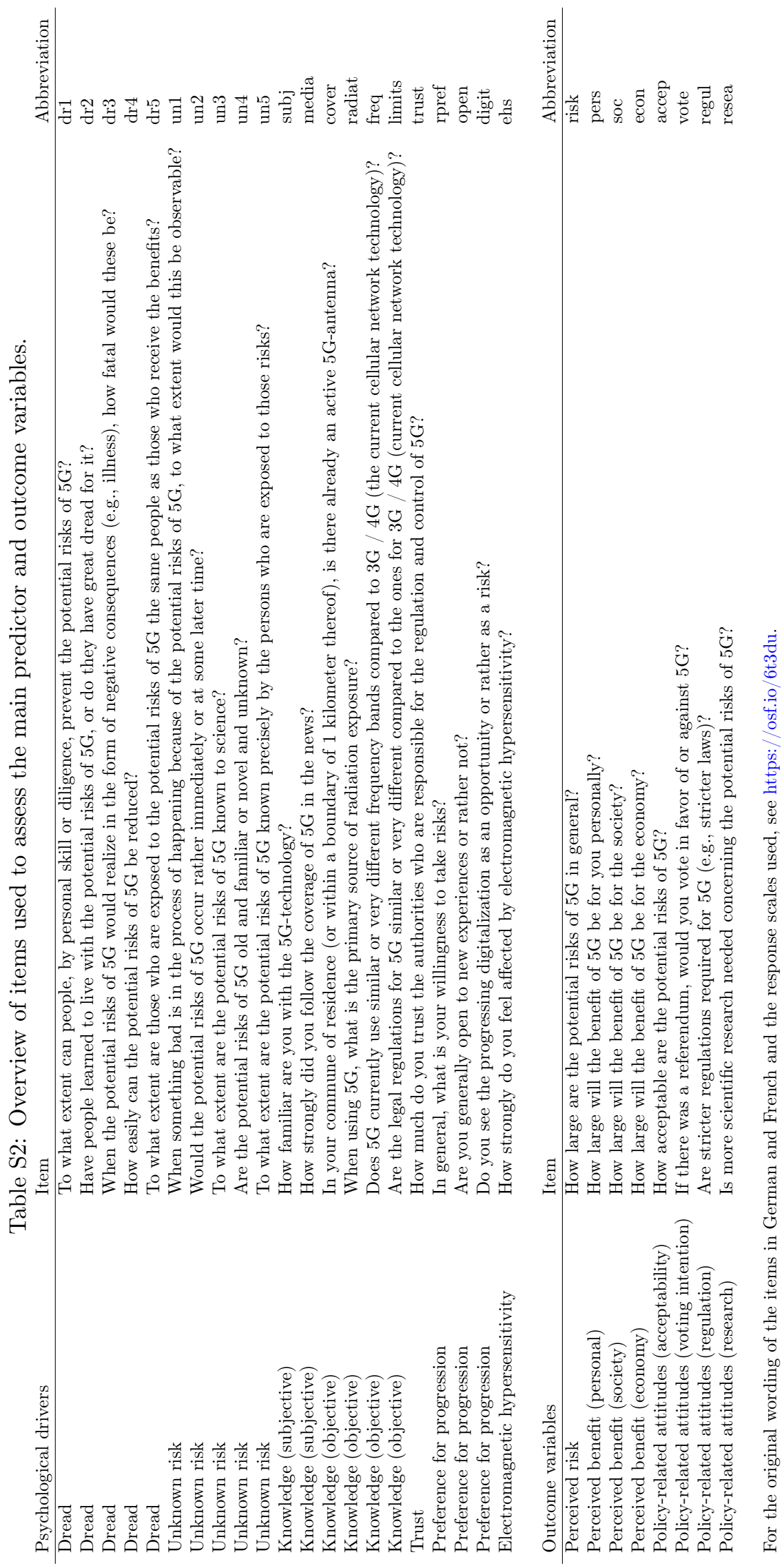




\section{References}

Alhakami, A. S., \& Slovic, P. (1994). A psychological study of the inverse relationship between perceived risk and perceived benefit. Risk Analysis, 14(6), 1085-1096. https://doi.org/10.1111/j.15396924.1994.tb00080.x

Brislin, R. W. (1970). Back-translation for cross-cultural research. Journal of Cross-Cultural Psychology, 1 (3), 185-216. https://doi.org/10.1177/135910457000100301

Clyde, M. A., Ghosh, J., \& Littman, M. L. (2011). Bayesian adaptive sampling for variable selection and model averaging. Journal of Computational and Graphical Statistics, 20(1), 80-101. https://doi.org/ $10.1198 /$ jcgs.2010.09049

Costa, P. T., \& MacCrae, R. R. (1992). Revised NEO personality inventory (NEO PI-R) and NEO five-factor inventory (NEO-FFI): Professional manual. Psychological Assessment Resources, Incorporated.

Epskamp, S., \& Stuber, w. c. f. S. (2017). semPlot: Path diagrams and visual analysis of various SEM packages' output. https://CRAN.R-project.org/package=semPlot

Finucane, M. L., Alhakami, A., Slovic, P., \& Johnson, S. M. (2000). The affect heuristic in judgments of risks and benefits. Journal of Behavioral Decision Making, 13(1), 1-17. https://doi.org/10.1002/(SICI) 1099-0771(200001/03)13:1<1::AID-BDM333>3.0.CO;2-S

Fischhoff, B., Slovic, P., Lichtenstein, S., Read, S., \& Combs, B. (1978). How safe is safe enough? A psychometric study of attitudes towards technological risks and benefits. Policy Sciences, 9(2), 127-152. https://doi.org/10.1007/BF00143739

Frey, R., Pedroni, A., Mata, R., Rieskamp, J., \& Hertwig, R. (2017). Risk preference shares the psychometric structure of major psychological traits. Science Advances, 3, e1701381. https://doi.org/10.1126/ sciadv. 1701381

Hinne, M., Gronau, Q. F., van den Bergh, D., \& Wagenmakers, E.-J. (2019). A conceptual introduction to Bayesian model averaging. PsyArXiv. https://doi.org/10.31234/osf.io/pfynb

$\mathrm{R}$ Core Team. (2019). R: A language and environment for statistical computing. Vienna, Austria. https: //www.R-project.org/

Rosseel, Y. (2012). Lavaan: An R package for structural equation modeling. Journal of Statistical Software, 48(2), 1-36. https://doi.org/10.18637/jss.v048.i02

Siegrist, M. (1999). A causal model explaining the perception and acceptance of gene technology. Journal of Applied Social Psychology, 29(10), 2093-2106. https://doi.org/10.1111/j.1559-1816.1999.tb02297.x

Siegrist, M. (2000). The influence of trust and perceptions of risks and benefits on the acceptance of gene technology. Risk Analysis, 20(2), 195-204. https://doi.org/10.1111/0272-4332.202020

Siegrist, M., Keller, C., Kastenholz, H., Frey, S., \& Wiek, A. (2007). Laypeople's and experts' perception of nanotechnology hazards. Risk Analysis, 27(1), 59-69. https://doi.org/10.1111/j.1539-6924.2006. 00859.x

Slovic, P., Fischhoff, B., \& Lichtenstein, S. (1985). Characterizing perceived risk. In R. W. Kates, C. Hohenemser, \& J. X. Kasperson (Eds.), Perilous Progress: Managing the Hazards of Technology (pp. 91125). Social Science Research Network. Retrieved September 11, 2019, from https://papers.ssrn. $\mathrm{com} /$ abstract $=2185557$

Stan Development Team. (2016). Rstanarm: Bayesian applied regression modeling via Stan. https://mcstan.org

van den Bergh, D., Clyde, M. A., Raj, A., de Jong, T., Gronau, Q. F., Marsman, M., Ly, A., \& Wagenmakers, E.-J. (2020). A tutorial on Bayesian multi-model linear regression with BAS and JASP. PsyArXiv. https://doi.org/10.31234/osf.io/pqju6

Weber, E. U., Blais, A. R., \& Betz, N. E. (2002). A domain-specific risk-attitude scale: Measuring risk perceptions and risk behaviors. Journal of Behavioral Decision Making, 15(4), 263-290. https://doi. $\operatorname{org} / 10.1002 / \mathrm{bdm} .414$ 